\title{
On next to soft threshold corrections to DIS and SIA processes
}

\section{A.H. Ajjath, Pooja Mukherjee, V. Ravindran, Aparna Sankar and Surabhi Tiwari}

The Institute of Mathematical Sciences, HBNI,

Taramani, Chennai 600113, India

E-mail: ajjathah@imsc.res.in, poojamukherjee@imsc.res.in, ravindra@imsc.res.in, aparnas@imsc.res.in, surabhit@imsc.res.in

ABSTRACT: We study the perturbative structure of threshold enhanced logarithms in the coefficient functions of deep inelastic scattering (DIS) and semi-inclusive $e^{+} e^{-}$annihilation (SIA) processes and setup a framework to sum them up to all orders in perturbation theory. Threshold logarithms show up as the distributions $\left((1-z)^{-1} \log ^{i}(1-z)\right)_{+}$from the soft plus virtual (SV) and as logarithms $\log ^{i}(1-z)$ from next to SV (NSV) contributions. We use the Sudakov differential and the renormalisation group equations along with the factorisation properties of parton level cross sections to obtain the resummed result which predicts SV as well as next to SV contributions to all orders in strong coupling constant. In Mellin $N$ space, we resum the large logarithms of the form $\log ^{i}(N)$ keeping $1 / N$ corrections. In particular, the towers of logarithms, each of the form $a_{s}^{n} / N^{\alpha} \log ^{2 n-\alpha}(N), a_{s}^{n} / N^{\alpha} \log ^{2 n-1-\alpha}(N) \cdots$ etc for $\alpha=0,1$, are summed to all orders in $a_{s}$.

KeYwords: Resummation, Perturbative QCD

ArXiv EPrint: 2007.12214 


\section{Contents}

1 Introduction 1

2 Next to $\mathrm{SV}$ in $z$ space $\quad 5$

3 All order predictions for $\Delta_{c} \quad 14$

4 Resummation in $N$ space 18

$5 \quad$ Physical evolution kernel 23

6 Conclusions 26

$\begin{array}{ll}\text { A NSV Partonic coefficient functions } \Delta_{c}^{N S V} & 27\end{array}$

$\begin{array}{llr}\text { B SV coefficients } \overline{\mathcal{G}}_{S V, i}^{c} \text { in } \Delta_{c} & 30\end{array}$

C NSV resummation constants $h_{i, j}^{c}(\omega) \quad 30$

D NSV resummation constants $\bar{g}_{i}^{c}(\omega) \quad 31$

\section{Introduction}

Radiative corrections to observables at high energy colliders are absolutely essential to understand the underlying quantum dynamics of the scattering events. There are a large number of accurate measurements already available from these colliders and they provide ample opportunity to investigate various theories that attempt to describe the physics. Theoretical predictions with unprecedented accuracy have already set stringent constraints on the parameters of the standard model (SM), also for many beyond the SM (BSM) scenarios. The observables that involve hadrons either in initial or in final state receive large perturbative and non-perturbative quantum corrections from strong interaction which is described by quantum chromodynamics (QCD). This is simply due to the strong coupling constant $\left(g_{s}\right)$ which is big and due to a large number of scattering channels that contribute. At high energies, thanks to factorisation properties of certain hadronic observables, which are infrared (IR) safe, the short distance perturbative part factorises from the non-perturbative one. This allows one to reliably compute the perturbative quantum corrections in powers of strong coupling constant $a_{s}=g_{s}^{2} / 16 \pi^{2}$ in QCD. The non-perturbative part of these IR safe observables are extracted in a process independent way. For example, the inclusive cross section of DIS of a lepton on a hadron factorises into perturbatively calculable coefficient functions $(\mathrm{CF})$ and parton distribution functions (PDF) that are non-perturbative in 
nature. The CFs are computed in powers of $a_{s}$ using the parton level scattering processes that contributes to the hadronic reaction. The PDFs are nothing but the probability of finding a parton inside the hadron during the scattering event, describing the long distance part of the hadronic events and hence can not be computed using perturbative techniques. However, being process independent, they can be extracted from an experiment and can be used for other experiments. Within QCD, they are defined in terms of hadronic matrix elements of certain gauge invariant quantum field theoretical operators made up of quark, anti-quark and gluon field operators. These matrix elements satisfy the renormalisation group (RG) equations which go by the name Altarelli-Parisi (AP) or DGLAP evolution equations. The corresponding anomalous dimensions are called AP splitting functions. Another example that is very similar to DIS is the production of a hadron in SIA of $e^{+} e^{-}$. One finds that scattering cross section in SIA also demonstrate factorisation of perturbatively calculable CFs and non-perturbative quantities called parton fragmentation functions (PFF). The CFs describe the production of a parton in the collision and the latter describes the fragmentation of the produced parton into a hadron. PFFs also satisfy AP or DGLAP equations with the corresponding AP splitting functions. Denoting $\sigma_{I}$ generically for the inclusive cross section for DIS $(I=D I S)$ and SIA $(I=S I A)$, the factorisation at high energies implies

$$
\sigma_{I}\left(Q^{2}, x_{I}\right)=\sigma_{I}^{(0)}\left(Q^{2}, \mu_{R}^{2}\right) \sum_{a=q, \bar{q}, g} \int d x f_{a}\left(\mu_{F}^{2}, x\right) \Delta_{I, a}\left(Q^{2}, \mu_{R}^{2}, \mu_{F}^{2}, z_{I}\right)+\mathcal{O}\left(1 / Q^{2}\right) .
$$

Since, we study these observables in the large $Q^{2}$ region, we drop the power suppressed contributions denoted by $\mathcal{O}\left(1 / Q^{2}\right)$ in the above formula and consider only the first term for rest of our study. $\sigma_{I}^{(0)}$ is the born level cross section and $\mu_{R}$ is the ultraviolet renormalisation scale, $f_{a}$ denotes PDF for $I=D I S$ and PFF for $I=S I A$. The PDFs depend on the partonic momentum fraction $x$ carried away from the hadron in DIS and PFFs depend on the hadronic momentum fraction that the hadron carries away from the parton. The scale $\mu_{F}$ is called the factorisation scale which separates perturbative and non-perturbative regions. The sum is over all the partons namely the quarks and anti-quarks of all flavours and the gluons. The scale $Q^{2}$ is the hard scale in the problem. For DIS, it is defined by $Q^{2}=-q^{2}, q$ being the momentum that is transferred from the incoming lepton to the target hadron. The corresponding scaling variable $x_{D I S}=Q^{2} / 2 P \cdot q$ where $P$ is the momentum of the target hadron. Similarly, for SIA, $Q^{2}=q^{2}$ with $q$ being the sum of momenta of incoming leptons and $x_{S I A}=2 P \cdot q / Q^{2}$, with $P$ being the momentum of hadron that fragments from the parton. The parton level scaling variables are $z_{D I S}=Q^{2} / 2 p \cdot q$ and $z_{S I A}=2 p \cdot q / Q^{2}$. Here, $p$ is the momentum of the parton in the respective scattering processes. In the rest of our paper, we drop $I$ in the argument of $\Delta_{I, a}$.

Perturbative QCD provides framework to compute $\Delta_{I, a}$ in powers of $a_{s}$ :

$$
\Delta_{I, a}\left(Q^{2}, \mu_{R}^{2}, \mu_{F}^{2}, z\right)=\sum_{i=0}^{\infty} a_{s}^{i}\left(\mu_{R}^{2}\right) \Delta_{I, a}^{(i)}\left(Q^{2}, \mu_{R}^{2}, \mu_{F}^{2}, z\right),
$$

where $\Delta_{I, a}^{(i)}$ at every order gets contribution from the parton level scattering processes. Beyond leading order in perturbation theory, at the intermediate stages they contain ul- 
traviolet (UV), soft and collinear divergences. The UV divergences go away when renormalisation of coupling, masses and fields are performed. The soft and collinear divergences are collectively called infrared (IR) divergences. The soft divergences come from zero momentum gluons in the loops of virtual contributions and real gluons in the gluon emission processes. The massless or light partons are responsible for collinear divergences. Thanks to KLN theorem [1, 2], soft and collinear divergences go away when the degenerate states that are responsible are summed at the partonic level. However, for DIS, the sum over degenerate partonic initial states are summed through convoluting them with bare PDFs. In practice, the initial state collinear divergences are factored out from the partonic subprocesses and then absorbed into the bare PDFs. This is called mass factorisation. Similar thing happens for the SIA where the final state collinear singularities are absorbed into bare PFFs to get IR safe observable. The factorisation scale quantifies the arbitrariness involved in the mass factorisation. In practice, both UV and IR divergences are regulated in dimensional regularisation by working in complex space time dimension $n=4+\epsilon$. The divergences show up as poles in $\epsilon$. The UV renormalisation and mass factorisation are done in modified minimal substraction $\overline{M S}$ scheme consistently. The inclusive cross sections for DIS are known to third order in QCD, see [3-5].

In CFs, the energy scales $Q^{2}, \mu_{R}^{2}$ and $\mu_{F}^{2}$ appear as logarithms and the partonic scaling variable shows up through $\delta(1-z)$, plus distributions $\mathcal{D}_{j}=\left(\log ^{j}(1-z) /(1-z)\right)_{+}$and regular functions of $z$ :

$$
\Delta_{I, a}(z)=\Delta_{I, a, \delta} \delta(1-z)+\sum_{j=0}^{\infty} \Delta_{I, a, \mathcal{D}_{j}}\left(\frac{\log ^{j}(1-z)}{1-z}\right)_{+}+\Delta_{I, a, R}(z),
$$

where we have suppressed the scales $Q^{2}, \mu_{R}^{2}$ and $\mu_{F}^{2}$ in the arguments of $\Delta_{I, a}$ and $\Delta_{I, a, Z}, Z=$ $\delta, \mathcal{D}_{j}, R$ on both sides. Large number of perturbative results provide opportunity to understand the universal structure of IR divergences. For example, the IR structure of multi-leg amplitudes in QCD is well understood beyond two loop level [6, 6-9] (see [10, 11] for a QFT with mixed gauge groups). In addition, we have large number of results for the inclusive cross sections that can shed light on the structure of $\Delta_{I, a, Z}$, see [12-14] for Higgs production and for invariant mass distribution of a pair of lepton in hadron colliders up to third order in QCD see [15-17], for complete list see [12, 16, 18-35] for Higgs production in gluon fusion and [15-17, 27-29, 33, 36-44] for Drell-Yan production.

The distributions $\delta(1-z)$ and $\mathcal{D}_{j}(z)$ result from the soft and collinear regions of the virtual and real emission diagrams. In the region where a scattering event involves infinite number of soft gluons each carrying almost zero momentum, the logarithms of the form $\log ^{i}(1-z) /(1-z)$ contribute to $\Delta_{I, a}$. This can happen in real emission scattering processes. These contributions are ill defined in 4 space-time dimensions in the limit $z \rightarrow 1$. The inclusion of these contribution gives the distributions $\mathcal{D}_{i}(z)$ and $\delta(1-z)$. The distributions that are present in $\Delta_{I, a}$ are called soft plus virtual (SV) contributions. SV results are available for many observables at colliders up to third order in QCD, see [2729, 43-48]. When the distributions are convoluted with PDFs or PFFs to obtain hadronic cross section, one finds that they not only dominate over other contributions but also are large at every order. Hence, they can spoil the reliability of the predictions from the 
truncated series. The resolution to this problem was successfully achieved in seminal works by Sterman [49] and Catani and Trentedue [50] through reorganisation of the perturbative series. It goes under the name threshold resummation, see also [51-56] for Higgs production in gluon fusion, [57, 58] for bottom quark annihilation, for DY [44, 52, 59-61] and for DIS and SIA of $e^{+} e^{-}$[62]. In Mellin space, the conjugate variable to $z$ is $N$ and the convolutions become normal products. Hence, the resummation is conveniently done in Mellin space. In the Mellin space, the threshold limit $z \rightarrow 1$ corresponds to large $N$. The large logarithms of $N$ at every order combined with the strong coupling constant can give order one contribution. Hence, the truncation of the series based on series expansion in $a_{s}$ is not allowed. However, thanks to factorisation properties, universality of IR contributions and renormalisation group invariance, we can systematically resum the order one terms, in particular, terms of the form $a_{s}\left(\mu_{R}^{2}\right) \beta_{0} \log (N)$ to all orders in perturbation theory. Defining $a_{s}\left(\mu_{R}^{2}\right) \beta_{0} \log (N)=\omega$, and treating $\omega$ to order 1 , following, [49, 50], we can organize

$$
\lim _{N \rightarrow \infty} \log \Delta_{I, a, N}=\log \tilde{g}_{0}^{I}\left(a_{s}\left(\mu_{R}^{2}\right)\right)+\log (N) g_{1}^{I}(\omega)+\sum_{i=0}^{\infty} a_{s}^{i}\left(\mu_{R}^{2}\right) g_{i+2}^{I}(\omega),
$$

where $\tilde{g}_{0}^{I}\left(a_{s}\left(\mu_{R}^{2}\right)\right)$ is $N$ independent. Inclusion of successive terms in eq. (1.4) predicts the leading logarithms (LL), next to leading (NLL) etc logarithms to all orders in $a_{s}$. The functions $g_{i}^{I}(\omega)$ depend on universal IR anomalous dimensions and $\tilde{g}_{0}^{I}$ depend on the hard process. For DIS, invariant mass distribution of lepton pairs in DY, Higgs boson productions in various channels, results for the resummation of threshold logarithms in $N$ space up to third order, namely next to next to next to leading logarithmic $\left(\mathrm{N}^{3} \mathrm{LL}\right)$ accuracy, are available $[44,52,58,61]$.

The resummed predictions played an important role to understand the experimental data in the threshold regions. However, the sub leading logarithms that are present in the regular part $\Delta_{I, a, R}(z)$ can not be ignored. We expand $\Delta_{I, a, R}(z)$ around $z=1$ to obtain

$$
\Delta_{I, a, R}(z)=\sum_{k=0}^{\infty} \Delta_{I, a, L}^{(k)} \log ^{k}(1-z)+\mathcal{O}(1-z)
$$

where the logarithms of the form $\log ^{k}(1-z), k=0,1, \cdots$ do contribute significantly at every order in perturbation theory near the threshold. We call them by next to SV (NSV) contributions. There have been several studies to understand the structure of NSV terms in the hadronic observables so that one can find whether the NSV terms can be systematically resummed to all orders like the way the SV terms are resummed. There have been several attempts to achieve this task. A remarkable development was made by Moch and Vogt in [63] (and $[31,35]$ ) using the second order results for DIS, semi-inclusive $e^{+} e^{-}$annihilation and Drell-Yan production of a pair of leptons in hadron collisions, and the physical evolution kernels to find the enhancement of a single-logarithms at large $z$ to all orders in $1-z$. The physical evolution kernel was exploited earlier in the work by [64]. It was found that the structure of corresponding leading $\log (1-z)$ terms in the kernel can be constrained [63] allowing them to predict certain next to SV logarithms at higher orders in $a_{s}$. The next to SV corrections to various inclusive processes were studied in a series of papers [65-73] 
and much progress have been made which lead to better understanding of the underlying physics. Recently some of us have studied inclusive production of pair of leptons in DrellYan process and of a Higgs boson in gluon fusion as well as in bottom quark annihilation in an attempt to resum these NSV terms to all orders [74]. We used factorisation properties and renormalisation group invariance along with the certain universal structure of real and virtual contributions using Sudakov $\mathrm{K}+\mathrm{G}$ equation to achieve this task. In this paper, we extend this to DIS and SIA to provide an all order result both in $z$ space and in $N$ space.

\section{Next to $\mathrm{SV}$ in $z$ space}

We begin with the unpolarised inclusive deep-inelastic lepton-nucleon scattering:

$$
l(k)+H(P) \rightarrow l\left(k^{\prime}\right)+X\left(P_{X}\right)
$$

where the incoming and scattered leptons $(l)$ carry the momenta $k$ and $k^{\prime}$ respectively, $H$ is the target hadron with the momentum $P$ and the $X$ is the set of inclusive final states with total momentum $P_{X}$. If we restrict to only photon exchange in the scattering, the inclusive cross section can be expressed in terms of two structure functions (SF) namely $F_{1}\left(Q^{2}, x\right)$ and $F_{2}\left(Q^{2}, x\right)$. The SFs are scalar functions and they parametrise the hadronic tensor $W_{\mu \nu}\left(Q^{2}, x\right)$ which carry the information of hadronic part of the DIS cross section. The tensor $W_{\mu \nu}$ is given by

$$
W_{\mu \nu}\left(Q^{2}, x\right)=\left(\frac{q_{\mu} q_{\nu}}{q^{2}}-g_{\mu \nu}\right) F_{1}\left(Q^{2}, x\right)-\frac{1}{2 x q^{2}}\left(q_{\mu}+2 x P_{\mu}\right)\left(q_{\nu}+2 x P_{\nu}\right) F_{2}\left(Q^{2}, x\right),
$$

where $q=k^{\prime}-k, Q^{2}=-q^{2}$ and the scaling variable, also called Björken $x$ is defined by $x=Q^{2} / 2 P \cdot q$. The hadronic tensor is related to Fourier transform of commutator of two electromagnetic currents sandwiched between the hadronic states. Due to the nonperturbative nature of the hadronic states, the structure functions are not computable in perturbation theory. However, in the Björken limit, thanks to operator product expansion, the hadronic tensor factorises into perturbatively calculable Wilson coefficients and nonperturbative composite operators sandwiched between hadronic states. Defining the Mellin moment of $F_{i}\left(Q^{2}, x\right)$ by

$$
F_{J, N}\left(Q^{2}\right)=\int_{0}^{1} d x x^{N-1} \frac{F_{J}\left(Q^{2}, x\right)}{x}, \quad J=2, L
$$

with $F_{L}=F_{2}-2 x F_{1}$ and computing them in the Bjöken limit, namely $Q^{2} \rightarrow \infty, P \cdot q \rightarrow \infty$ keeping $x=Q^{2} / 2 P \cdot q$ fixed, one finds

$$
F_{J, N}\left(Q^{2}\right)=\sum_{a=n s, q, g} C_{J, a, N}\left(Q^{2}, \mu_{F}^{2}\right) \mathcal{A}_{a, N}\left(\mu_{F}^{2}\right)
$$

where $n s$ denotes the non-singlet combination of quark operators, which does not mix with the gluonic operator under UV renormalisation and the indices $q$ and $g$ correspond to those operators which mix among themselves. The matrix element of local operators denoted by $\mathcal{A}_{a, N}$ are not calculable using perturbative methods. However, their evolution in terms of 
the scale $\mu_{F}$ is controlled by the perturbatively calculable AP splitting functions through AP evolution equations. The Wilson coefficients $C_{J, a, N}$ are computed in powers of strong coupling constant.

In QCD improved parton model, one can relate the local operators $\mathcal{A}_{a, N}$ to Mellin moments of appropriate combinations of PDFs and the Wilson coefficients $C_{J, a, N}$ to parton level coefficient functions (CFs). The Wilson coefficients, equivalently CFs can be computed, within the framework of perturbative QCD, order by order in strong coupling constant using parton level subprocesses. The contributions, beyond the leading order, contain UV, soft and collinear divergences. If we regulate them in dimensional regularisation, the UV divergences arise as poles in $\epsilon$ and are removed in modified minimal subtraction $(\overline{M S})$ scheme. As we discussed in the introduction, the soft and collinear divergences resulting from final state partons cancel independently after summing up the contributions from all possible degenerate states. However, the collinear divergences arising from the initial state light partons remain. Those are removed at the hadronic level through a procedure called mass factorisation.

In the following, we consider the Wilson coefficients equivalent to the CFs that contribute to a generic DIS scattering process. We denote them by $\Delta_{c}$ where the index $c=q, \bar{q}, g$. The factorisation allows us to relate the CFs, $\Delta_{c}$ and the parton level subprocesses through the mass factorisation given as

$$
\frac{1}{z} \hat{\sigma}_{c}\left(Q^{2}, z, \epsilon\right)=\sigma^{(0)}\left(\mu_{R}^{2}\right) \Gamma_{c^{\prime} c}\left(z, \mu_{F}^{2}, \epsilon\right) \otimes\left(\frac{1}{z} C_{c^{\prime}}\left(Q^{2}, \mu_{R}^{2}, \mu_{F}^{2}, z, \epsilon\right)\right) .
$$

In eq. (2.5), $\hat{\sigma}_{c}\left(Q^{2}, z, \epsilon\right) / z$ is the appropriate UV finite parton level cross section computed in space time dimension $n=4+\epsilon$. The scaling variable $z$ is given by $z=Q^{2} / 2 p \cdot q$, where $p$ is the momentum of the incoming parton in the scattering event. The function $\Gamma_{c^{\prime} c}$ is the Altarelli-Parisi (AP) [75] kernel which contains the collinear divergences of $\hat{\sigma}_{c}$ in $\overline{M S}$ scheme. As in [74], we limit ourselves to SV+NSV contributions to CFs, which means that we drop those terms in $C_{c}$ that vanish when $z \rightarrow 1$ and call the resulting ones by $\Delta_{c}$. For the quark/anti-quark initiated processes in DIS with photon exchange, gluon initiated one with Higgs boson exchange, the infrared singluar partonic cross sections can be factorised into squares of UV renormalisation constant, $Z_{U V, c}^{2}$, of form factor (FF), $\left|\hat{F}_{c}\right|^{2}$ and a function $\mathcal{S}_{J}^{c}$ that is sensitive to real radiations. This is always possible as $Z_{U V, c}^{2}$ and $\left|\hat{F}_{c}\right|^{2}$ are simply proportional to $\delta(1-z)$ and can be factored out from these partonic channels. That is,

$$
\begin{aligned}
z^{-1} \hat{\sigma}_{c}\left(Q^{2}, z, \epsilon\right)= & \sigma^{(0)}\left(\mu_{R}^{2}\right)\left(Z_{U V, c}\left(\hat{a}_{s}, \mu_{R}^{2}, \mu^{2}, \epsilon\right)\right)^{2}\left|\hat{F}_{c}\left(\hat{a}_{s}, \mu^{2}, Q^{2}, \epsilon\right)\right|^{2} \\
& \times \delta(1-z) \otimes \mathcal{S}_{J}^{c}\left(\hat{a}_{s}, \mu^{2}, Q^{2}, z, \epsilon\right)
\end{aligned}
$$

As it will be shown in the following, the function $\mathcal{S}_{J}^{c}$ satisfies a differential equation which admits a solution namely the convoluted exponential of $\Phi_{c}$. That is,

$$
\mathcal{S}_{J}^{c}=\mathcal{C} \exp \left(2 \Phi_{J}^{c}\left(\hat{a}_{s}, \mu^{2}, Q^{2}, z, \epsilon\right)\right)
$$


Substituting for $\hat{\sigma}_{c}$ from (2.6) in terms of $\Phi_{J}^{c}$ in (2.5), we obtain

$$
\Delta_{c}\left(Q^{2}, \mu_{R}^{2}, \mu_{F}^{2}, z\right)=\left.\mathcal{C} \exp \left(\Psi_{J}^{c}\left(Q^{2}, \mu_{R}^{2}, \mu_{F}^{2}, z, \epsilon\right)\right)\right|_{\epsilon=0},
$$

where the function $\Psi_{J}^{c}$ is given by

$$
\begin{aligned}
& \Psi_{J}^{c}\left(Q^{2}, \mu_{R}^{2}, \mu_{F}^{2}, z, \epsilon\right)=\left(\ln \left(Z_{U V, c}\left(\hat{a}_{s}, \mu^{2}, \mu_{R}^{2}, \epsilon\right)\right)^{2}+\ln \left|\hat{F}_{c}\left(\hat{a}_{s}, \mu^{2}, Q^{2}, \epsilon\right)\right|^{2}\right) \delta(1-z) \\
& +2 \Phi_{J}^{c}\left(\hat{a}_{s}, \mu^{2}, Q^{2}, z, \epsilon\right)-\mathcal{C} \ln \Gamma_{c c}\left(\hat{a}_{s}, \mu^{2}, \mu_{F}^{2}, z, \epsilon\right) .
\end{aligned}
$$

The symbol $\otimes$ represents the Mellin convolution. The operation of $\mathcal{C}$ on any given function is defined in eq. (2) of [45]. In this expression, $c=q$ (quark/antiquark) for photon-exchange DIS, and $c=g$ (gluon) for Higgs-exchange DIS. Though the constituents of $\Psi_{J}^{c}$ contains UV and IR divergent terms, the sum of all these terms is finite and is regular in the variable $\epsilon$. It contains the distributions such as $\delta(1-z), \mathcal{D}_{j}(z)$ and the logarithms of the form $\log ^{i}(1-z), i=0,1, \cdots$. In eq. (2.9), the overall renormalisation constant for DIS via the photon exchange is one to all orders in QCD. For DIS via the Higgs boson exchange, $Z_{U V, c}$ is equivalent to that of Higgs-gluon effective operator [45].

The AP kernels that remove collinear divergences from the parton level cross sections are solutions to AP evolution equation (see eq. (2.11) in [74]) which are controlled by AP splitting functions $P_{a b}\left(\mu_{F}^{2}, z\right)$. They contain convolutions of AP spitting functions. In the above equation, we have kept only diagonal part of AP kernel $\Gamma_{a b}$ and dropped the non-diagonal AP kernels. We explain the reason below. Consider $\Delta_{q}$ in photon-exchange DIS. It gets contributions from three different terms namely $\hat{\sigma}_{q} \otimes \Gamma_{q q}, \hat{\sigma}_{\bar{q}} \otimes \Gamma_{\bar{q} q}$ and $\hat{\sigma}_{g} \otimes \Gamma_{g q}$. The non-diagonal AP kernels and $\hat{\sigma}_{g}$ contain only NSV and/or beyond NSV terms. Upon convolution, the terms $\hat{\sigma}_{\bar{q}} \otimes \Gamma_{\bar{q} q}$ and $\hat{\sigma}_{g} \otimes \Gamma_{g q}$ will give only beyond NSV terms. In addition, only diagonal parts of splitting functions $P_{a b}\left(z, \mu_{F}^{2}\right)$ in $\Gamma_{a b}\left(z, \mu_{F}^{2}, \epsilon\right)$ need to be kept as the contributions from convolutions of two or more non-diagonal splitting functions give only beyond NSV terms. The diagonal $P_{c c}\left(z, \mu_{F}^{2}\right)$ are expanded around $z=1$ and all those terms that do not contribute to $\mathrm{SV}+\mathrm{NSV}$ are eliminated. The diagonal AP splitting functions near $z=1$ take the following form:

$$
P_{c c}\left(z, a_{s}\left(\mu_{F}^{2}\right)\right)=2 B^{c}\left(a_{s}\left(\mu_{F}^{2}\right)\right) \delta(1-z)+2 P_{c c}^{\prime}\left(z, a_{s}\left(\mu_{F}^{2}\right)\right)
$$

where,

$$
P_{c c}^{\prime}\left(z, a_{s}\left(\mu_{F}^{2}\right)\right)=\left[A^{c}\left(a_{s}\left(\mu_{F}^{2}\right)\right) \mathcal{D}_{0}(z)+C^{c}\left(a_{s}\left(\mu_{F}^{2}\right)\right) \log (1-z)+D^{c}\left(a_{s}\left(\mu_{F}^{2}\right)\right)\right] .
$$

The constants $C^{c}$ and $D^{c}$ can be obtained from the splitting functions $P_{c c}$ which are known to three loops in QCD [76, 77] (see [4, 76-84] for the lower order ones).

For the DIS with the photon exchange, the interaction of virtual photon from the lepton with the target hadron is through a vector current. Hence, the FF that contributes to the inclusive cross section is the square of the quark matrix element for the vector current. 
Vector current being conserved does not get any overall UV renormalisation and hence $Z_{U V, c}$ is identity. If the exchange particle is the scalar Higgs boson and its interaction with the hadron is through an operator which is not conserved, then $Z_{U V, c}$ will be non-zero. For example, in order to compute singlet splitting functions, one resorts to scattering of a scalar Higgs boson on a gluon target and the interaction between them is governed by effective operator $G_{\mu \nu}^{a} G^{\mu \nu, a} \phi$. Here $G_{\mu \nu}^{a}$ is the gluon field strength operator and $\phi$ is the Higgs boson field. The coupling of Higgs boson and the gluon through this composite operator requires addition overall renormalisation and hence $Z_{U V, c}$ [85] is included in eq. (2.9). FFs in general are computable in regularised QCD perturbation theory in powers of strong coupling constant. FFs are known in QCD up to third order in perturbation theory, [86-98]. Both UV and IR divergences appear as poles in $\epsilon$ and they demonstrate rich IR structure, and satisfy differential equations such as RG equation, $\mu_{R}^{2} \frac{d}{d \mu_{R}^{2}} \hat{F}_{c}=0$ and Sudakov differential equation [45, 99-105]. The latter is called $\mathrm{K}+\mathrm{G}$ equation. It is used to study their IR structure of FFs in terms of IR anomalous dimensions such as cusp $A^{c}$,collinear $B^{c}$ and soft $f^{c}$ anomalous dimensions. The perturbative structure of FFs provides valuable information of the underlying quantum field theory and it was exploited to understand the structure of multi-leg on-shell amplitudes in QCD [6,6-9] (see [10, 11] for a QFT with mixed gauge groups) and they are found to be helpful to understand the IR structure of real emission processes [28, 43, 45-47].

In $[28,45]$, using the $\mathrm{K}+\mathrm{G}$ structure of $\mathrm{FF}$ and the finiteness of inclusive cross sections, it was shown that the soft distribution functions $\mathcal{S}_{J}^{c}$ equivalently, $\Phi_{J}^{c}$ in Drell-Yan production of lepton pairs and production of Higgs boson in gluon fusion in hadron colliders and soft plus jet function in DIS processes were shown to satisfy $\mathrm{K}+\mathrm{G}$ type differential equations. The infrared structure of these functions can be understood in terms of the IR anomalous dimensions. In particular, the threshold logarithms that contribute in the soft and collinear regions of the real emission processes are contained in these soft functions. The universal nature of these contributions are due to the IR anomalous dimensions. The fact that these contributions exponentiate, owing to the $\mathrm{K}+\mathrm{G}$ differential equation that they satisfy, the all order predictions as well as the resummation of threshold effects are possible. In the present case, our task is to find a suitable $\mathrm{K}+\mathrm{G}$ equation which can capture not only SV contributions but also NSV contributions.

Using the fact that the function $\mathcal{S}_{J}^{c}$ given in (2.6) can be factorised from the rest of the contributions and that the $\mathrm{FF}$ satisfies $\mathrm{K}+\mathrm{G}$ equation, we can easily show that $\mathcal{S}_{J}^{c}$ also satisfies a $\mathrm{K}+\mathrm{G}$ type differential equation. Note that $\mathcal{S}_{J}^{c}$ captures both soft and next to soft contributions. Since the $\mathrm{K}+\mathrm{G}$ equation corresponding to $\mathcal{S}_{J}^{c}$ admits a solution of convoluted exponential form, we have expressed $\mathcal{S}_{J}^{c}=\mathcal{C} \exp \left(\Phi_{J}^{c}\right)$ as given in (2.7), where the real emission contributions, normalised by $\left|\hat{F}^{c}\right|^{2}$ and $Z_{U V, c}^{2}$ are encapsulated in the function $\Phi_{J}^{c}$. Here, the exponential form of the real emission contributions holds true for both SV and NSV cases as the factorisation and the $\mathrm{K}+\mathrm{G}$ differential equation are valid for all $z$. We can use the finiteness of the coefficient function, $\Delta_{c}$ to determine $\mathcal{S}_{J}^{c}$ order by order in perturbation theory. In summary, we find that $\mathcal{S}_{J}^{c}$, equivalently $\Phi_{J}^{c}$ satisfies $\mathrm{K}+\mathrm{G}$ type equation with the kernels $\bar{K}_{J}^{c}$ and $\bar{G}_{J}^{c}$ which contain right IR divergences and 
the finite terms respectively:

$$
Q^{2} \frac{d}{d Q^{2}} \Phi_{\mathrm{J}}^{c}=\frac{1}{2}\left[\bar{K}_{J}^{c}\left(\hat{a}_{s}, \frac{\mu_{R}^{2}}{\mu^{2}}, \epsilon, z\right)+\bar{G}_{J}^{c}\left(\hat{a}_{s}, \frac{Q^{2}}{\mu_{R}^{2}}, \frac{\mu_{R}^{2}}{\mu^{2}}, \epsilon, z\right)\right] .
$$

Note that both $\bar{K}_{J}^{c}$ and $\bar{G}_{J}^{c}$ that control the evolution of $\Phi_{J}^{c}$ are dependent on $z$. In addition, following the structure of $\mathrm{K}+\mathrm{G}$ equation for the $\mathrm{FF}$, we keep all the IR divergent terms in $\bar{K}_{J}^{c}$ and move the entire $Q^{2}$ dependence along with IR finite terms to $\bar{G}_{J}^{c}$. This is possible to all orders thanks to the factorisation property of real emission contributions. Following [28, 45], we find the solution to eq. (2.12). Expanding both $\bar{K}_{J}^{c}$ and $\bar{G}_{J}^{c}$ in powers of bare coupling constant $\hat{a}_{s}$ and integrating over $Q^{2}$, we find

$$
\Phi_{\mathrm{J}}{ }^{c}\left(\hat{a}_{s}, Q^{2}, \mu^{2}, z, \epsilon\right)=\sum_{i=1}^{\infty} \hat{a}_{s}^{i}\left(\frac{Q^{2}(1-z)}{\mu^{2} z}\right)^{i \frac{\epsilon}{2}} S_{\epsilon}^{i}\left(\frac{i \epsilon}{2(1-z)}\right) \hat{\phi}_{c}^{(i)}(z, \epsilon) .
$$

Few comments on the solution are in order. The solution satisfies RG equation, namely $\mu_{R}^{2} \frac{d}{d \mu_{R}^{2}} \Phi_{J}^{c}=0$ which organises the perturbative expansion in such a way that after UV renormalisation, $\Phi_{J}^{c}$ is free of UV divergences. In addition, it controls the structure of logarithms of $Q^{2}$ through the term $Q^{i \epsilon}$. Hence, $\Phi_{J}^{c}$ contains only IR divergences and they are organised in such a way that they cancel against those from the FF and AP kernel. The factor $((1-z) / z)^{\epsilon / 2}$ is inspired from the two body phase of the next to leading order DIS scattering and the term $1 /(1-z)$ results form the dominant contribution of the square of the parton level cross section in the limit $z \rightarrow 1$. The regular function denoted by $\hat{\phi}_{c}^{(i)}(z, \epsilon)$ determines the SV as well as NSV terms systematically when it is expanded around $z=1$. We determine the entire IR divergences in $\bar{K}_{J}^{c}$ from the those of $K_{J}^{c}$ of FF and of the SV part of the AP kernel by demanding IR finiteness of the SV part of the $\Delta_{c}$. The remaining collinear divergences present in the AP kernel, which are sensitive to NSV terms, determine $\bar{G}_{J}^{c}$ with the condition of IR finiteness of $\Delta_{c}$ implied.

For convenience, we decompose $\Phi_{J}^{c}$ as $\Phi_{J}^{c}=\Phi_{J, A}^{c}+\Phi_{J, B}^{c}$ in such a way that $\Phi_{J, A}^{c}$ contains only SV terms $i . e$ all the distributions $\mathcal{D}_{j}$ and $\delta(1-z)$ and $\Phi_{J, B}^{c}$ contains NSV terms namely $\log ^{k}(1-z), k=0,1, \cdots$ in the limit $z \rightarrow 1$. An all order solution for $\Phi_{J, A}^{c}$ in powers of $\hat{a}_{s}$ in dimensional regularisation is given in [45] and we reproduce here for completeness:

$$
\Phi_{J, A}^{c}\left(\hat{a}_{s}, Q^{2}, \mu^{2}, \epsilon, z\right)=\sum_{i=1}^{\infty} \hat{a}_{s}^{i}\left(\frac{Q^{2}(1-z)}{\mu^{2}}\right)^{i \frac{\epsilon}{2}} S_{\epsilon}^{i}\left(\frac{i \epsilon}{2(1-z)}\right) \hat{\phi}_{S V}^{c(i)}(\epsilon),
$$

where,

$$
\hat{\phi}_{S V}^{c(i)}(\epsilon)=\frac{1}{i \epsilon}\left[\bar{K}_{J}^{c(i)}(\epsilon)+\bar{G}_{J, S V}^{c(i)}(\epsilon)\right] .
$$

The constants $\bar{K}_{J}^{c(i)}(\epsilon)$ and $\bar{G}_{J, S V}^{c(i)}(\epsilon)$ are given in eq. (35) and eq. (37) of [28] respectively and they are known up to third order in perturbation theory [28, 45, 106-112]. The distributions in $\Phi_{J}^{c}$ are related to Jet functions which are building blocks in Soft-Collinear effective theory (SCET) [113-118] which captures the physics of soft and collinear dynamics of high energy scattering processes through the soft and jet functions. The jet functions 
describe the propagation of collinear partons inside jets. In SCET, the quark and gluon jet functions have been computed to higher orders in perturbation theory [106-111]. Alternatively, as was shown in [112], they can be extracted from the coefficient functions of DIS with photon and Higgs exchanges [3, 4]. Noting that the finite part of $\Phi_{J}^{c}$ is nothing but the logarithm of Jet function, three loop contribution to gluon jet function was obtained in [112].

The solution $\Phi_{J, B}^{c}$ that contains NSV part of the $\Phi_{J}^{c}$ takes the following form:

$$
\Phi_{J, B}^{c}\left(\hat{a}_{s}, \mu^{2}, Q^{2}, z, \epsilon\right)=\sum_{i=1}^{\infty} \hat{a}_{s}^{i} S_{\epsilon}^{i}\left(\frac{Q^{2}(1-z)}{\mu^{2}}\right)^{i \frac{\epsilon}{2}} \frac{1}{2} \bar{\varphi}_{c}^{(i)}(z, \epsilon) .
$$

We obtain this solution by setting $\bar{K}_{J}^{c}$ to zero and replacing $\bar{G}_{J}^{c}$ by $\bar{G}_{J}^{c}-\bar{G}_{J, S V}^{c}$ in eq. (2.12) as they were already taken into account to obtain SV part of the solution. The functions $\bar{\varphi}_{c}^{(i)}$ contain both UV and IR divergences as poles in $\epsilon$. The former goes away when the coupling constant renormalisation is performed. As the entire soft divergences of real emission processes are contained in $\Phi_{J, A}^{c}$, the coefficients $\bar{\varphi}_{c}^{(i)}(z, \epsilon)$ will have only collinear divergences that will exactly cancel with those of AP kernel. The finite part of it can be determined by comparing against $\Delta_{c}$ order by order in perturbation theory. We split $\bar{\varphi}_{c}^{(i)}$ as a sum of collinear divergent and collinear finite coefficients as

$$
\bar{\varphi}_{c}^{(i)}(z, \epsilon)=\bar{\varphi}_{s, c}^{(i)}(z, \epsilon)+\bar{\varphi}_{f, c}^{(i)}(z, \epsilon) .
$$

From the finiteness of $\Delta_{c}$ and NSV part of AP kernel, we find that the singular coefficients $\bar{\varphi}_{s, c}^{(i)}$ is identical to $\bar{K}_{c}^{(i)}$ given in eq. (35) of [28] with the following replacement of $A^{c}$ by $L^{c}$ :

$$
\bar{\varphi}_{s, c}^{(i)}(z, \epsilon)=\left.\bar{K}_{J}^{c(i)}(\epsilon)\right|_{A^{c} \rightarrow L^{c}(z)},
$$

where $L^{c}\left(a_{s}\left(\mu_{R}^{2}\right), z\right)$ is finite and can be expanded in powers of $a_{s}\left(\mu_{R}^{2}\right)$ as

$$
L^{c}\left(a_{s}\left(\mu_{R}^{2}\right), z\right)=\sum_{i=1}^{\infty} a_{s}^{i}\left(\mu_{R}^{2}\right) L_{i}^{c}(z)
$$

The coefficients $\bar{\varphi}_{f, c}^{(i)}(z, \epsilon)$ are determined from NSV terms of $\Delta_{c}$ at every order in perturbation theory. Although we can determine soft and collinear divergences present in $\Phi_{J}^{c}$ at every order in perturbation theory using FF, AP kernel but the finite part requires the explicit computation of real emission subprocesses around $z=1$. Note that $\Delta_{c}$ are known to third order for several observables in perturbation theory and they allow us to extract the finite part of $\Phi_{J}^{c}$ up to third order. In order to determine the finite part, we express the series expansions $\Phi_{J, A}^{c}$ and $\Phi_{J, B}^{c}$ given in eq. (2.14) and eq. (2.16) respectively as

$$
\begin{aligned}
\Phi_{J, A}^{c}\left(\hat{a}_{s}, \mu^{2}, Q^{2}, z, \epsilon\right)= & \left(\frac{1}{2(1-z)}\left\{\int_{\mu_{F}^{2}}^{Q^{2}(1-z)} \frac{d \lambda^{2}}{\lambda^{2}} A^{c}\left(a_{s}\left(\lambda^{2}\right)\right)+\bar{G}_{J, S V}^{c}\left(a_{s}\left(Q^{2}(1-z)\right), \epsilon\right)\right\}\right)_{+} \\
& +\delta(1-z) \sum_{i=1}^{\infty} \hat{a}_{s}^{i}\left(\frac{Q^{2}}{\mu^{2}}\right)^{i \frac{\epsilon}{2}} S_{\epsilon}^{i} \phi_{S V}^{c(i)}(\epsilon) \\
& +\frac{1}{2(1-z)_{+}} \sum_{i=1}^{\infty} \hat{a}_{s}^{i}\left(\frac{\mu_{F}^{2}}{\mu^{2}}\right)^{i \frac{\epsilon}{2}} S_{\epsilon}^{i} \bar{K}_{J}^{c(i)}(\epsilon) .
\end{aligned}
$$


where $\bar{G}_{J, S V}^{c}\left(a_{s}\left(Q^{2}(1-z)\right), \epsilon\right)$ are related to the threshold exponent $\mathbf{B}_{D I S}^{I}\left(a_{s}\left(Q^{2}(1-z)\right)\right)$ via eq. (48) of $[28]$ and

$$
\begin{aligned}
\Phi_{J, B}^{c}\left(\hat{a}_{s}, \mu^{2}, Q^{2}, z, \epsilon\right)= & \frac{1}{2} \int_{\mu_{F}^{2}}^{Q^{2}(1-z)} \frac{d \lambda^{2}}{\lambda^{2}} L^{c}\left(a_{s}\left(\lambda^{2}\right), z\right)+\left.\bar{\varphi}_{f, c}\left(a_{s}\left(Q^{2}(1-z)\right), z, \epsilon\right)\right|_{\epsilon=0} \\
& +\bar{\varphi}_{s, c}\left(a_{s}\left(\mu_{F}^{2}\right), z, \epsilon\right)
\end{aligned}
$$

where,

$$
\bar{\varphi}_{a, c}\left(a_{s}\left(\lambda^{2}\right), z\right)=\sum_{i=1}^{\infty} \hat{a}_{s}^{i}\left(\frac{\lambda^{2}}{\mu^{2}}\right)^{i \frac{\epsilon}{2}} S_{\epsilon}^{i} \frac{1}{2} \bar{\varphi}_{a, c}^{(i)}(z, \epsilon) . \quad a=f, s
$$

In the expression given in eq. (2.21) the first line is finite when $\epsilon \rightarrow 0$ whereas second line is divergent in the same limit. The RG invariance of $\Phi_{J, B}^{c}$ implies that $\bar{\varphi}_{s, c}$ satisfies the renormalisation group equation:

$$
\mu_{F}^{2} \frac{d}{d \mu_{F}^{2}} \bar{\varphi}_{s, c}\left(a_{s}\left(\mu_{F}^{2}\right), z\right)=L^{c}\left(a_{s}\left(\mu_{F}^{2}\right), z\right) .
$$

The anomalous dimension $L^{c}$ can be determined by demanding finiteness of $\Delta_{c}$ and it turns out that it is half of NSV part of the AP splitting functions (see [74]), that is

$$
L^{c}\left(a_{s}, z\right)=C^{c}\left(a_{s}\right) \log (1-z)+D^{c}\left(a_{s}\right) .
$$

Note that the SV part of the diagonal splitting function in the logarithms of diagonal AP kernel in eq. (2.9) cancels the one from $\bar{G}_{J, S V}^{c}$ and the remaining divergence coming from NSV part cancels against $\bar{\varphi}_{s, c}$ making $\Delta_{c}$ finite to all orders in $a_{s}$. This is guaranteed by the factorisation of collinear divergences to all orders.

Having understood the structure of the singular part $\bar{\varphi}_{s, c}$, we now focus on the finite part $\bar{\varphi}_{f, c}$. The finite part $\bar{\varphi}_{f, c}$ is parametrised in terms of $\log ^{k}(1-z)$ as:

$$
\bar{\varphi}_{f, c}\left(a_{s}\left(Q^{2}(1-z)\right), z\right)=\sum_{i=1}^{\infty} a_{s}^{i}\left(Q^{2}(1-z)\right) \sum_{k=0}^{i} \frac{1}{2} \bar{\varphi}_{c, i}^{(k)} \log ^{k}(1-z) .
$$

The coefficients $\bar{\varphi}_{c, i}^{(k)}$ in eq. (2.25) are related to the constants $\mathcal{G}_{L, i}^{c,(j, k)} \mathrm{s}$ through

$$
\begin{aligned}
\bar{\varphi}_{c, 1}^{(k)}= & \mathcal{G}_{L, 1}^{c,(1, k)}, \quad k=0,1 \\
\bar{\varphi}_{c, 2}^{(k)}= & \frac{1}{2} \mathcal{G}_{L, 2}^{c,(1, k)}+\beta_{0} \mathcal{G}_{L, 1}^{c,(2, k)}, k=0,1,2 \\
\bar{\varphi}_{c, 3}^{(k)}= & \frac{1}{3} \mathcal{G}_{L, 3}^{c,(1, k)}+\frac{2}{3} \beta_{1} \mathcal{G}_{L, 1}^{c,(2, k)}+\frac{2}{3} \beta_{0} \mathcal{G}_{L, 2}^{c,(2, k)}+\frac{4}{3} \beta_{0}^{2} \mathcal{G}_{L, 1}^{c,(3, k)}, \quad k=0,1,2,3 \\
\bar{\varphi}_{c, 4}^{(k)}= & \frac{1}{4} \mathcal{G}_{L, 4}^{c,(1, k)}+\frac{1}{2} \beta_{2} \mathcal{G}_{L, 1}^{c,(2, k)}+\frac{1}{2} \beta_{1} \mathcal{G}_{L, 2}^{c,(2, k)}+\frac{1}{2} \beta_{0} \mathcal{G}_{L, 3}^{c,(2, k)}+2 \beta_{0} \beta_{1} \mathcal{G}_{L, 1}^{c,(3, k)}+\beta_{0}^{2} \mathcal{G}_{L, 2}^{c,(3, k)} \\
& +2 \beta_{0}^{3} \mathcal{G}_{L, 1}^{c,(4, k)}, \quad k=0,1,2,3,4
\end{aligned}
$$

with $\mathcal{G}_{L, 1}^{c,(2,3)}, \mathcal{G}_{L, 1}^{c,(2,4)}, \mathcal{G}_{L, 2}^{c,(2,4)}, \mathcal{G}_{L, 1}^{c,(3,4)}$ not contributing to $\bar{\varphi}_{c, i}^{(k)}$. In the above equations, $\mathcal{G}_{L, i}^{c,(j, k)}(z)$ are expansion coefficients of $\mathcal{G}_{L, i}^{c}(z, \epsilon)$ defined by $\bar{G}_{J, L}^{c}=\bar{G}_{J}^{c}-\bar{G}_{J, S V}^{c}$ :

$$
\bar{G}_{J, L}^{c}\left(\hat{a}_{s}, \frac{Q^{2}}{\mu_{R}^{2}}, \frac{\mu_{R}^{2}}{\mu^{2}}, z, \epsilon\right)=\sum_{i=1}^{\infty} a_{s}^{i}\left(Q^{2}(1-z)\right) \sum_{j=0}^{\infty} \sum_{k=0}^{i+j-1} \mathcal{G}_{L, i}^{c,(j, k)} \log ^{k}(1-z)
$$


In the dimensionally regularised theory, given the order of perturbation namely the power of $a_{s}$ and the accuracy of $\epsilon$, the loop integrals in the virtual diagrams and phase space integrals for the real emission sub-processes demonstrate a systematic logarithmic structure. For example, the highest power of $\log (1-z)$ of the coefficient of $a_{s}^{i} \epsilon^{j}$ in a perturbative expansion, is controlled by both $i$ and $j$. For the inclusive reactions that we considered, we find that the highest power of $\log (1-z)$ is always less than or equal to $i+j-1$. Hence, the summation over $k$ in the eq. (2.27) runs from 0 to $i+j-1$. This translates to the upper limit $i$ in the summation over $k$ in eq. (2.25). At every order $a_{s}^{i}$, the coefficients $\mathcal{G}_{L, i}^{c,(j, k)}$ or their combination namely $\bar{\varphi}_{c, i}^{(k)}$ for various value of $i$ and $k$ can be extracted from explicit perturbative results of $\Delta_{c}$.

So far, we studied how NSV terms can be systematically included in the threshold expansion of inclusive cross section of DIS. Same methodology can be applied for the SIA as well to obtain the corresponding all order result. Noting that the SIA is time like process, namely the energy scale $Q^{2}$ is negative of its center of mass energy and that the collinear factorisation requires time-like splitting functions, we can obtain $\tilde{\Psi}_{J}^{c}$ (see eq. (2.9) for SIA by replacing $Q^{2}$ in $\hat{F}_{c}$ by $-q^{2}, q^{2}>0, Q^{2}$ in $\Phi_{J}^{c}$ by $q^{2}$ and the splitting functions in $\Gamma_{c c}$ by the time like ones:

$$
\begin{aligned}
\hat{F}_{c}\left(\hat{a}_{s}, \mu^{2}, Q^{2}, \epsilon\right) & \rightarrow \hat{F}_{c}\left(\hat{a}_{s}, \mu^{2},-q^{2}, \epsilon\right) \\
\Phi_{J}^{c}\left(\hat{a}_{s}, \mu^{2}, Q^{2}, \epsilon\right) & \rightarrow \tilde{\Phi}_{J}^{c}\left(\hat{a}_{s}, \mu^{2}, q^{2}, \epsilon\right) \\
\ln \Gamma_{c c}\left(\hat{a}_{s}, \mu^{2}, \mu_{F}^{2}, \epsilon\right) & \rightarrow \ln \tilde{\Gamma}_{c c}\left(\hat{a}_{s}, \mu^{2}, \mu_{F}^{2}, \epsilon\right)
\end{aligned}
$$

where $\tilde{\Gamma}_{c c}$ is the time like AP kernel. The solution for jet function for the SIA, $\tilde{\Phi}_{J}^{c}$ is obtained exactly the way we obtained $\Phi_{J}^{c}$. The complete result for the SV part can be found [119] which we will not repeat here. For the NSV part of $\tilde{\Phi}_{J}^{c}$, the function $\tilde{\Phi}_{J, B}^{c}$ is found to be the same as eq. (2.16) with the replacements $\bar{\varphi}_{a, c} \rightarrow \tilde{\varphi}_{a, c}$ and consequently $\bar{\varphi}_{a, c} \rightarrow \tilde{\varphi}_{a, c}$ with $a=s, f$ and $Q^{2} \rightarrow q^{2}$.

The coefficient functions $\Delta_{c}$ for DIS via the exchange of a photon as well as a Higgs boson are available up to third order in $[3,4]$ respectively. One can find the analytical results for FFs, overall renormalisation constants, the functions $\Phi_{J, A}^{c}$ and $\Gamma_{c \bar{c}}$ up to third order in the literature. Following $[63,120]$, we define the non singlet DIS structure functions as:

$$
\mathcal{F}_{1}=2 F_{1, n s}, \quad \mathcal{F}_{2}=\frac{1}{x} F_{2, n s}, \quad \mathcal{F}_{3}=F_{3}{ }^{\nu+\bar{\nu}}
$$

Now using the available results up to third order for $c=q$ (photon-exchange DIS) for the structure functions $\mathcal{F}_{1}$ and $\mathcal{F}_{3}$, we found the functions $\bar{\varphi}_{q, i}^{(k)}$ as,

$$
\begin{array}{lll}
\bar{\varphi}_{q, 1}^{(0)}=10 C_{F}, & \bar{\varphi}_{q, 1}^{(1)}=0, & \bar{\varphi}_{q, 2}^{(1)}=10 C_{F} C_{A}-22 C_{F}^{2}, \\
\bar{\varphi}_{q, 2}^{(2)}=-4 C_{F}^{2}, & \bar{\varphi}_{q, 3}^{(3)}=C_{F}^{2} C_{A}\left(-\frac{176}{27}\right)+n_{f} C_{F}^{2}\left(\frac{32}{27}\right) . &
\end{array}
$$

and for the structure function $\mathcal{F}_{2}$,

$$
\begin{aligned}
& \bar{\varphi}_{q, 1}^{(0)}=14 C_{F}, \quad \bar{\varphi}_{q, 1}^{(1)}=0, \\
& \bar{\varphi}_{q, 2}^{(0)}=C_{F} C_{A}\left(\frac{5734}{27}-64 \zeta_{3}-\frac{32}{3} \zeta_{2}\right)-C_{F}^{2}\left(\frac{25}{2}+44 \zeta_{2}-72 \zeta_{3}\right)+n_{f} C_{F}\left(-\frac{1060}{27}+\frac{8}{3} \zeta_{2}\right),
\end{aligned}
$$




$$
\begin{aligned}
\bar{\varphi}_{q, 2}^{(1)}= & C_{F} C_{A}\left(-6+16 \zeta_{2}\right)+C_{F}^{2}\left(26-32 \zeta_{2}\right), \quad \bar{\varphi}_{q, 2}^{(2)}=-4 C_{F}^{2} \\
\bar{\varphi}_{q, 3}^{(0)}= & C_{F} C_{A}^{2}\left(\frac{3231470}{729}+72 \zeta_{5}-\frac{63128}{27} \zeta_{3}-\frac{46208}{81} \zeta_{2}-\frac{64}{3} \zeta_{2} \zeta_{3}+\frac{2324}{15} \zeta_{2}^{2}\right) \\
& +C_{F}^{2} C_{A}\left(-\frac{83255}{54}+1320 \zeta_{5}+\frac{22180}{9} \zeta_{3}-\frac{25984}{27} \zeta_{2}+272 \zeta_{2} \zeta_{3}-\frac{6464}{15} \zeta_{2}^{2}\right) \\
& +C_{F}^{3}\left(\frac{1319}{6}-2000 \zeta_{5}+444 \zeta_{3}-\frac{50}{3} \zeta_{2}-224 \zeta_{2} \zeta_{3}+\frac{9056}{15} \zeta_{2}^{2}\right)+n_{f} C_{F} C_{A}\left(-\frac{972940}{729}\right. \\
& \left.+236 \zeta_{3}+\frac{21068}{81} \zeta_{2}-\frac{304}{15} \zeta_{2}^{2}\right)+n_{f} C_{F}^{2}\left(-\frac{133}{3}-\frac{880}{3} \zeta_{3}+\frac{2896}{27} \zeta_{2}+\frac{256}{5} \zeta_{2}^{2}\right) \\
& +n_{f}^{2} C_{F}\left(\frac{68312}{729}+\frac{32}{27} \zeta_{3}-\frac{592}{27} \zeta_{2}\right)+\left[\frac{d a b c^{2}}{n}\right] f l_{11}\left(-128+1280 \zeta_{5}-704 \zeta_{3}-448 \zeta_{2}\right. \\
& \left.+128 \zeta_{2} \zeta_{3}+\frac{64}{5} \zeta_{2}^{2}\right), \\
\bar{\varphi}_{q, 3}^{(1)}= & C_{F} C_{A}^{2}\left(-\frac{5680}{9}+\frac{376}{3} \zeta_{3}+\frac{1792}{3} \zeta_{2}-\frac{128}{5} \zeta_{2}^{2}\right)+C_{F}^{2} C_{A}\left(+\frac{95612}{81}+\frac{1400}{3} \zeta_{3}-1004 \zeta_{2}\right. \\
& \left.-\frac{512}{5} \zeta_{2}^{2}\right)+C_{F}^{3}\left(\frac{134}{3}-720 \zeta_{3}-\frac{160}{3} \zeta_{2}+\frac{1536}{5} \zeta_{2}^{2}\right)+n_{f} C_{F} C_{A}\left(\frac{892}{9}-\frac{160}{3} \zeta_{3}-\frac{260}{3} \zeta_{2}\right) \\
& +n_{f} C_{F}^{2}\left(-\frac{16136}{81}+\frac{128}{3} \zeta_{3}+144 \zeta_{2}\right), \\
\bar{\varphi}_{q, 3}^{(2)}= & C_{F} C_{A}^{2}\left(\frac{14}{3}-32 \zeta_{3}+58 \zeta_{2}\right)-C_{F}^{2} C_{A}\left(94-128 \zeta_{3}+\frac{316}{3} \zeta_{2}\right)+C_{F}^{3}\left(\frac{496}{3}-128 \zeta_{3}\right) \\
& +n_{f} C_{F} C_{A}\left(2-\frac{16}{3} \zeta_{2}\right)+n_{f} C_{F}^{2}\left(\frac{4}{3}+\frac{32}{3} \zeta_{2}\right), \\
\bar{\varphi}_{q, 3}^{(3)}= & C_{F}^{2} C_{A}\left(-\frac{176}{27}\right)+n_{f} C_{F}^{2}\left(\frac{32}{27}\right),
\end{aligned}
$$

Similarly, the non singlet time-like transverse $\left(\mathcal{F}_{T}\right)$ and longitudinal $\left(\mathcal{F}_{L}\right)$ structure functions in SIA (see $[63,119,120])$ are defined as,

$$
\mathcal{F}_{T}=F_{T, n s}, \quad \mathcal{F}_{L}=F_{L, n s},
$$

and $\tilde{\varphi}_{q, i}^{(k)}$ for $\mathcal{F}_{\mathcal{T}}$ are found to be

$$
\begin{array}{lll}
\tilde{\varphi}_{q, 1}^{(0)}=-8 C_{F}, & \tilde{\varphi}_{q, 1}^{(1)}=0, & \tilde{\varphi}_{q, 2}^{(1)}=-10 C_{F} C_{A}+22 C_{F}^{2}, \\
\tilde{\varphi}_{q, 2}^{(2)}=4 C_{F}^{2}, & \tilde{\varphi}_{q, 3}^{(3)}=C_{F}^{2} C_{A}\left(\frac{176}{27}\right)-n_{f} C_{F}^{2}\left(\frac{32}{27}\right) . &
\end{array}
$$

and for $\mathcal{F}_{L}, \tilde{\varphi}_{q, i}^{(k)}$ are found to be

$$
\begin{array}{ll}
\tilde{\varphi}_{q, 1}^{(0)}=2 C_{F} & \tilde{\varphi}_{q, 1}^{(1)}=4 C_{F} \\
\tilde{\varphi}_{q, 2}^{(1)} & =C_{F} C_{A}\left(\frac{328}{9}\right)+C_{F}^{2}\left(48-16 \zeta_{2}\right)-C_{F} n_{f}\left(\frac{64}{9}\right), \\
\tilde{\varphi}_{q, 2}^{(2)} & =\frac{22}{3} C_{F} C_{A}+8 C_{F}^{2}-C_{F} n_{f}\left(\frac{4}{3}\right), \\
\tilde{\varphi}_{q, 3}^{(3)} & =C_{F} C_{A}^{2}\left(\frac{484}{27}\right)+\frac{88}{3} C_{A} C_{F}^{2}-C_{A} C_{F} n_{f}\left(\frac{176}{27}\right)-\frac{16}{3} C_{F}^{2} n_{f}+n_{f}^{2} C_{F}\left(\frac{16}{27}\right) .
\end{array}
$$


and for $c=g$ (Higgs-exchange DIS) the CFs to the gluon structure function $F_{\varphi}$ gives the following $\bar{\varphi}_{g, i}^{(k)}$,

$$
\begin{aligned}
& \bar{\varphi}_{g, 1}^{(0)}=\frac{1}{3} C_{A}+\frac{2}{3} n_{f}, \quad \bar{\varphi}_{g, 1}^{(1)}=0, \\
& \bar{\varphi}_{g, 2}^{(2)}=-4 C_{A}^{2}, \quad \bar{\varphi}_{g, 3}^{(3)}=C_{A}^{3}\left(-\frac{176}{27}\right)+n_{f} C_{A}^{2}\left(\frac{32}{27}\right) . \\
& \bar{\varphi}_{g, 2}^{(1)}=-14 C_{A}^{2}+2 n_{f} C_{A},
\end{aligned}
$$

Here, the constants $C_{A}=N_{c}$ and $C_{F}=\left(N_{c}^{2}-1\right) / 2 N_{c}$ are Casimirs of $S U\left(N_{c}\right)$ gauge group and $n_{f}$ is number of active flavours. The result for color factor $\left[\frac{d a b c^{2}}{N_{c}}\right] f l_{11}$ can be found in [3]. For $\mathcal{F}_{1}$ and $\mathcal{F}_{3}$, we could not obtain all the constants $\bar{\varphi}_{f, i}^{(k)}$ as the results for $\Delta_{q}$ corresponding to them are not available in the literature. Also for fragmentation functions, we have given only those that are possible to extract from the available CFs of fragmentation functions. Hence in conclusion, the coefficients $\bar{\varphi}_{f, q}$ given in eqs. (2.30), (2.31) along with the NSV part of the AP splitting functions determine $\Phi_{J, B}^{q}$ up to third order in $a_{s}$. Note that $\Phi_{J, A}^{q}$ is already known $[28,106-112]$ to the same accuracy. This completes the determination of $\Phi_{J}^{c}$ to third order in perturbation theory.

Having obtained $\Phi_{J}^{c}$ to third order, we make few observations. The structure of SV part of $\Phi_{J}^{c}$, namely $\Phi_{J, A}^{c}$, is well understood in terms of the cusp anomalous dimension $A^{c}$ and the function $\bar{G}_{J, S V}^{c}$. In particular, one finds that the entire SV part of $\Phi_{J}^{c}$ is universal as it is independent of the hard interaction. In the present case, this means that $\Phi_{J, A}^{c}$ is same for all the structure functions. However, it depends only on the parton that participates in the hard scattering. For photon-DIS, quark and anti-quarks are the ones that interact directly with the photon and hence the index $c=q, \bar{q}$ in cusp anomalous dimension and $\bar{G}_{J, S V}^{c}$. For the Higgs-DIS, both the cusp anomalous dimension as well as $\bar{G}_{J, S V}^{c}$ will depend on the gluon and hence they will be different from those of photonDIS. Unlike the SV part, NSV part does not have universal structure even though part of NSV contains process independent anomalous dimensions $C^{c}$ and $D^{c}$ resulting from AP splitting functions. From eqs. (2.30), (2.31), we find that the explicit results on $\bar{\varphi}_{f, c}$ extracted for different structure functions do not coincide, implying that they are sensitive to hard scattering of quarks/anti-quarks with the photon. In [74], some of us studied the NSV contributions to production of lepton pairs in Drell-Yan and production of Higgs boson in bottom quark annihilation and found that the corresponding $\varphi_{f, q}$ and $\varphi_{f, b}$ differ at third order hinting towards the breakdown of universality for the NSV part.

\section{All order predictions for $\Delta_{c}$}

In the earlier section we discussed extensively about each of the building blocks which constitute the master formula given in eq. (2.9). We have also shown that these building blocks satisfy certain differential equations which in turn is controlled by universal anomalous dimensions. Now in this section we aim to discuss the predictability of the solutions to the governing differential equations. For example, differential equation corresponding to RG can help us to predict logarithms of $\mu_{R}^{2}$. Similarly AP equations predict logarithms 
of $\mu_{F}^{2}$ and $\mathrm{K}+\mathrm{G}$ equations of $\mathrm{FF}$ and $\Phi_{J}^{c}$ predict threshold contributions $\delta(1-z), \mathcal{D}_{i}$ and $\log ^{k}(1-z), k=0,1, \cdots$ at higher orders in $a_{s}$. Hence, the knowledge of $\Phi_{J}^{c}$, FFs for the quark/gluon and the AP kernel $\Gamma_{c c}$, all known to third order can be used to predict certain $\mathrm{SV}$ as well as NSV terms in $\Delta_{c}$ beyond third order. Let us expand the CF $\Delta_{c}$ in powers of $a_{s}\left(\mu_{R}^{2}\right)$ as

$$
\Delta_{c}\left(Q^{2}, \mu_{R}^{2}, \mu_{F}^{2}, z\right)=\sum_{i=0}^{\infty} a_{s}^{i}\left(\mu_{R}^{2}\right) \Delta_{c}^{(i)}\left(Q^{2}, \mu_{R}^{2}, \mu_{F}^{2}, z\right) .
$$

where the coefficient $\Delta_{c}^{(i)}$ can be determined from eq. (2.8)-(2.9). Note that $\Delta_{c}^{(0)}=\delta(1-z)$. By definition, $\Delta_{c}$ contains only SV and NSV terms and hence terms of order $\mathcal{O}((1-$ $\left.z)^{\alpha}\right), \alpha>0$ are dropped.

Using the definition of $\mathcal{C}$, we first expand the exponential of $\Psi_{J}^{c}$ in eq. (2.8) in powers of $a_{s}\left(\mu_{R}^{2}\right)$ and then perform all the convolutions. This gives, at each order in perturbation theory, a tower of SV terms, such as the distributions $\mathcal{D}_{i}, i=0,1, \cdots$ and $\delta(1-z)$ and of next to SV terms namely the logarithms $\log ^{i}(1-z), i=0,1, \cdots$.

If $\Psi_{J}^{c}$ is known to $a_{s}$, the master formula eq. (2.8) can predict the leading SV terms $\left(\mathcal{D}_{3}, \mathcal{D}_{2}\right),\left(\mathcal{D}_{5}, \mathcal{D}_{4}\right), \cdots,\left(\mathcal{D}_{2 i-1}, \mathcal{D}_{2 i-2}\right)$ and the leading NSV terms $\log ^{3}(1-z), \log ^{5}(1-$ $z), \cdots, \log ^{2 i-1}(1-z)$ at $a_{s}^{2}, a_{s}^{3}, \cdots, a_{s}^{i}$ respectively for all $i$. Note that $C_{1}^{c}$ is identically zero and hence $\log ^{2 i}(1-z)$ terms do not contribute irrespective of $i$. Similarly the knowledge of $\Psi_{J}^{c}$ to order $a_{s}^{2}$ can predict the tower of distributions $\left(\mathcal{D}_{3}, \mathcal{D}_{2}\right),\left(\mathcal{D}_{5}, \mathcal{D}_{4}\right), \cdots,\left(\mathcal{D}_{2 i-3}, \mathcal{D}_{2 i-4}\right)$ and of $\log ^{4}(1-z), \log ^{6}(1-z), \cdots, \log ^{2 i-2}(1-z)$ at $a_{s}^{3}, a_{s}^{4}, \cdots, a_{s}^{i}$ respectively for all $i$. DIS results for the photon exchange are known to $a_{s}^{3}$ and it allows us to confirm our predictions at second and third orders based on the knowledge of $\Phi_{J}^{c}$ at $a_{s}$ and at $a_{s}^{2}$ respectively. We also confirmed our predictions for SV and NSV terms at third order against those given in $[3,4]$ using the $\Phi_{J}^{c}$ known to order $a_{s}$. This explains the all order predictive nature of eq. (2.8). The complete knowledge of $\Phi_{J}^{c}$ up to third order can be used to predict certain SV and NSV terms at fourth order for $\Delta_{q}$ because the former allows us to predict a tower of $\left(\mathcal{D}_{3}, \mathcal{D}_{2}\right),\left(\mathcal{D}_{5}, \mathcal{D}_{4}\right) \cdots,\left(\mathcal{D}_{2 i-5}, \mathcal{D}_{2 i-6}\right)$ and of $\log ^{5}(1-z), \log ^{7} \cdots, \log ^{2 i-3}(1-z)$ at $a_{s}^{4}, a_{s}^{5}, \cdots, a_{s}^{i}$ respectively for all $i$.

In the following, we present our predictions for the NSV terms $L_{z}$ till seventh order in $a_{s}$. For the DIS structure function $\mathcal{F}_{1}$, we find

$$
\begin{aligned}
\Delta_{q, 1}^{N S V}= & a_{s} \Delta_{q, 1}^{N S V(1)}+a_{s}^{2} \Delta_{q, 1}^{N S V(2)}+a_{s}^{3} \Delta_{q, 1}^{N S V(3)}+a_{s}^{4}\left[\left\{-\frac{16}{3} C_{F}^{4}\right\} L_{z}^{7}+\left\{\frac{308}{9} C_{F}^{3} C_{A}\right.\right. \\
& \left.+\frac{232}{3} C_{F}^{4}-\frac{56}{9} n_{f} C_{F}^{3}\right\} L_{z}^{6}-\left\{\frac{1936}{27} C_{F}^{2} C_{A}^{2}+\left(\frac{16384}{27}-48 \zeta_{2}\right) C_{F}^{3} C_{A}\right. \\
& \left.\left.+\left(188-128 \zeta_{2}\right) C_{F}^{4}-\frac{704}{27} n_{f} C_{F}^{2} C_{A}-\frac{2920}{27} n_{f} C_{F}^{3}+\frac{64}{27} n_{f}^{2} C_{F}^{2}\right\} L_{z}^{5}+\mathcal{O}\left(L_{z}^{4}\right)\right] \\
& +a_{s}^{5}\left[\left\{-\frac{8}{3} C_{F}^{5}\right\} L_{z}^{9}+\left\{-\frac{16}{3} C_{F}^{4} n_{f}+\frac{88}{3} C_{F}^{4} C_{A}+\frac{148}{3} C_{F}^{5}\right\} L_{z}^{8}+\left\{-\frac{320}{81} C_{F}^{3} n_{f}^{2}\right.\right. \\
& +\frac{3520}{81} C_{F}^{3} C_{A} n_{f}-\frac{9680}{81} C_{F}^{3} C_{A}^{2}+\frac{3056}{27} C_{F}^{4} n_{f}+\left(-\frac{17216}{27}+\frac{128}{3} \zeta_{2}\right) C_{F}^{4} C_{A} \\
& \left.\left.+\left(-\frac{532}{3}+\frac{320}{3} \zeta_{2}\right) C_{F}^{5}\right\} L_{z}^{7}+\mathcal{O}\left(L_{z}^{6}\right)\right]+a_{s}^{6}\left[\left\{-\frac{16}{15} C_{F}^{6}\right\} L_{z}^{11}+\left\{-\frac{88}{27} C_{F}^{5} n_{f}\right.\right. \\
& \left.+\frac{484}{27} C_{F}^{5} C_{A}+24 C_{F}^{6}\right\} L_{z}^{10}+\left\{-\frac{320}{81} C_{F}^{4} n_{f}^{2}+\frac{3520}{81} C_{F}^{4} C_{A} n_{f}-\frac{9680}{81} C_{F}^{4} C_{A}^{2}\right.
\end{aligned}
$$




\begin{tabular}{|l|l|l|l||l|l|l|}
\hline \multicolumn{3}{|c||}{ GIVEN } & \multicolumn{4}{|c|}{ PREDICTIONS } \\
\hline$\Psi_{c}^{(1)}$ & $\Psi_{c}^{(2)}$ & $\Psi_{c}^{(3)}$ & $\Psi_{c}^{(n)}$ & $\Delta_{c}^{(2)}$ & $\Delta_{c}^{(3)}$ & \multicolumn{1}{|c|}{$\Delta_{c}^{(3)}$} \\
\hline $\mathcal{D}_{0}, \mathcal{D}_{1}, \delta$ & & & & $\mathcal{D}_{3}, \mathcal{D}_{2}$ & $\mathcal{D}_{5}, \mathcal{D}_{4}$ & $\mathcal{D}_{(2 i-1)}, \mathcal{D}_{(2 i-2)}$ \\
$L_{z}^{1}, L_{z}^{0}$ & & & & $L_{z}^{3}$ & $L_{z}^{5}$ & $L_{z}^{(2 i-1)}$ \\
\hline & & & & & $\mathcal{D}_{3}, \mathcal{D}_{2}$ & $\mathcal{D}_{(2 i-3)}, \mathcal{D}_{(2 i-4)}$ \\
& $\mathcal{D}_{0}, \mathcal{D}_{1}, \delta$ & & & $L_{z}^{4}$ & $L_{z}^{(2 i-2)}$ \\
\hline & $L_{z}^{2}, L_{z}^{1}, L_{z}^{0}$ & & & & & $\mathcal{D}_{(2 i-5)}, \mathcal{D}_{(2 i-6)}$ \\
& & $\mathcal{D}_{0}, \mathcal{D}_{1}, \delta$ & & & & $L_{z}^{(2 i-3)}$ \\
\hline & & $L_{z}^{3}, \cdots, L_{z}^{0}$ & & & & $\mathcal{D}_{(2 i-(2 n-1))}, \mathcal{D}_{(2 i-2 n)}$ \\
& & & $\mathcal{D}_{0}, \mathcal{D}_{1}, \delta$ & & & $L_{z}^{(2 i-n)}$ \\
\hline & & & $L_{z}^{n}, \cdots, L_{z}^{0}$ & & & \\
\hline
\end{tabular}

Table 1. Towers of Distributions $\left(\mathcal{D}_{i}\right)$ and NSV logarithms $\left(\log ^{i}(1-z)\right)$ that can be predicted for $\Delta_{c}$ using eq. (2.8). Here $\Psi_{c}^{(i)}$ and $\Delta_{c}^{(i)}$ denotes $\Psi_{c}$ and $\Delta_{c}$ at order $a_{s}^{i}$ respectively. Also the symbol $L_{z}^{i}$ denotes $\log ^{i}(1-z)$.

$$
\begin{aligned}
& \left.\left.+\frac{6632}{81} C_{F}^{5} n_{f}+\left(-\frac{37376}{81}+\frac{80}{3} \zeta_{2}\right) C_{F}^{5} C_{A}+\left(-\frac{344}{3}+64 \zeta_{2}\right) C_{F}^{6}\right\} L_{z}^{9}+\mathcal{O}\left(L_{z}^{8}\right)\right] \\
& +\boldsymbol{a}_{s}^{7}\left[\left\{-\frac{16}{45} C_{F}^{7}\right\} L_{z}^{13}+\left\{-\frac{208}{135} C_{F}^{6} n_{f}+\frac{1144}{135} C_{F}^{6} C_{A}+\frac{424}{45} C_{F}^{7}\right\} L_{z}^{12}\right. \\
& +\left\{-\frac{224}{81} C_{F}^{5} n_{f}^{2}+\frac{2464}{81} C_{F}^{5} C_{A} n_{f}-\frac{6776}{81} C_{F}^{5} C_{A}^{2}+\frac{18128}{405} C_{F}^{6} n_{f}+\left(-\frac{20416}{81}\right.\right. \\
& \left.\left.\left.+\frac{64}{5} \zeta_{2}\right) C_{F}^{6} C_{A}+\left(-\frac{844}{15}+\frac{448}{15} \zeta_{2}\right) C_{F}^{7}\right\} L_{z}^{11}+\mathcal{O}\left(L_{z}^{10}\right)\right]
\end{aligned}
$$

for the DIS structure function $\mathcal{F}_{2}$,

$$
\begin{aligned}
\Delta_{q, 2}^{N S V}= & a_{s} \Delta_{q, 2}^{N S V(1)}+a_{s}^{2} \Delta_{q, 2}^{N S V(2)}+a_{s}^{3} \Delta_{q, 2}^{N S V(3)}+a_{s}^{4}\left[\Delta_{q, 1}^{N S V(4)}+\left\{\frac{16}{3} C_{F}^{4}\right\} L_{z}^{6}-\left\{\frac{728}{9} C_{F}^{3} C_{A}\right.\right. \\
& \left.\left.-\frac{80}{9} n_{f} C_{F}^{3}-32 \zeta_{2} C_{F}^{3} C_{A}+\left(-72+64 \zeta_{2}\right) C_{F}^{4}\right\} L_{z}^{5}+\mathcal{O}\left(L_{z}^{4}\right)\right]+\boldsymbol{a}_{\boldsymbol{s}}^{5}\left[\Delta_{q, 1}^{N S V(5)}+\left\{\frac{8}{3} C_{F}^{5}\right\} L_{z}^{8}\right. \\
& \left.+\left\{\left(-\frac{544}{9}+\frac{64}{3} \zeta_{2}\right) C_{F}^{4} C_{A}+\frac{64}{9} C_{F}^{4} n_{f}+\left(48-\frac{128}{3} \zeta_{2}\right) C_{F}^{5}\right\} L_{z}^{7}+\mathcal{O}\left(L_{z}^{6}\right)\right]+ \\
& a_{\boldsymbol{s}}^{\mathbf{6}}\left[\Delta_{q, 1}^{N S V(6)}+\left\{\frac{16}{15} C_{F}^{6}\right\} L_{z}^{10}+\left\{\left(-\frac{904}{27} C_{F}^{5} C_{A}+\frac{32}{3} \zeta_{2}\right) C_{F}^{5} C_{A}+\frac{112}{27} C_{F}^{5} n_{f}+(24\right.\right. \\
& \left.\left.\left.-\frac{64}{3} \zeta_{2}\right) C_{F}^{6}\right\} L_{z}^{9}+\mathcal{O}\left(L_{z}^{8}\right)\right]+\boldsymbol{a}_{\boldsymbol{s}}^{7}\left[\Delta_{q, 1}^{N S V(7)}+\left\{\frac{16}{45} C_{F}^{7}\right\} L_{z}^{12}+\left\{\left(-\frac{1984}{135}+\frac{64}{15} \zeta_{2}\right) C_{F}^{6} C_{A}\right.\right. \\
& \left.\left.+\frac{256}{135} C_{F}^{6} n_{f}+\left(\frac{48}{5} C_{F}^{7}-\frac{128}{15} \zeta_{2}\right) C_{F}^{7}\right\} L_{z}^{11}+\mathcal{O}\left(L_{z}^{10}\right)\right],
\end{aligned}
$$

for the SIA transverse structure function $\mathcal{F}_{T}$,

$$
\begin{aligned}
\tilde{\Delta}_{q, T}^{N S V}= & a_{s} \tilde{\Delta}_{q, T}^{N S V(1)}+a_{s}^{2} \tilde{\Delta}_{q, T}^{N S V(2)}+a_{s}^{3} \tilde{\Delta}_{q, T}^{N S V(3)}+\boldsymbol{a}_{\boldsymbol{s}}^{\mathbf{4}}\left[\Delta_{q, 1}^{N S V(4)}-72 C_{F}^{4} L_{z}^{6}\right. \\
& \left.+\left\{\frac{10316}{27} C_{F}^{3} C_{A}+\left(292-96 \zeta_{2}\right) C_{F}^{4}-\frac{2072}{27} n_{f} C_{F}^{3}\right\} L_{z}^{5}+\mathcal{O}\left(L_{z}^{4}\right)\right]+\boldsymbol{a}_{\boldsymbol{s}}^{\mathbf{5}}\left[\Delta_{q, 1}^{N S V(5)}\right. \\
& \left.-44 C_{F}^{5} L_{z}^{8}+\left\{\frac{11072}{27} C_{F}^{4} C_{A}-\frac{2144}{27} C_{F}^{4} n_{f}+\left(\frac{728}{3}-64 \zeta_{2}\right) C_{F}^{5}\right\} L_{z}^{7}+\mathcal{O}\left(L_{z}^{6}\right)\right]
\end{aligned}
$$




$$
\begin{aligned}
& +\boldsymbol{a}_{\boldsymbol{s}}^{\mathbf{6}}\left[\Delta_{q, 1}^{N S V(6)}-\left\{\frac{104}{5} C_{F}^{6}\right\} L_{z}^{10}+\left\{\frac{24484}{81} C_{F}^{5} C_{A}-\frac{4648}{81} C_{F}^{5} n_{f}+\left(\frac{436}{3}\right.\right.\right. \\
& \left.\left.\left.-32 \zeta_{2}\right) C_{F}^{6}\right\} L_{z}^{9}+\mathcal{O}\left(L_{z}^{8}\right)\right]+\boldsymbol{a}_{\boldsymbol{s}}^{7}\left[\Delta_{q, 1}^{N S V(7)}-8 C_{F}^{7} L_{z}^{12}+\left\{\frac{67888}{405} C_{F}^{6} C_{A}\right.\right. \\
& \left.\left.-\frac{12736}{405} C_{F}^{6} n_{f}+\left(\frac{1016}{15}-\frac{64}{5} \zeta_{2}\right) C_{F}^{7}\right\} L_{z}^{11}+\mathcal{O}\left(L_{z}^{10}\right)\right]
\end{aligned}
$$

and for the SIA longitudinal structure function $\mathcal{F}_{L}$,

$$
\begin{aligned}
\tilde{\Delta}_{q, L}^{N S V}= & a_{s} \tilde{\Delta}_{q, L}^{N S V(1)}+a_{s}^{2} \tilde{\Delta}_{q, L}^{N S V(2)}+a_{s}^{3} \tilde{\Delta}_{q, L}^{N S V(3)}+\boldsymbol{a}_{\boldsymbol{s}}^{\mathbf{4}}\left[\left(\frac{8}{3} C_{F}^{4}\right) L_{z}^{6}-\left\{\left(\frac{364}{9}-16 \zeta_{2}\right) C_{F}^{3} C_{A}\right.\right. \\
& \left.\left.+\left(32 \zeta_{2}-36\right) C_{F}^{4}-\frac{40}{9} n_{f} C_{F}^{3}\right\} L_{z}^{5}+\mathcal{O}\left(L_{z}^{4}\right)\right]+\boldsymbol{a}_{\boldsymbol{s}}^{5}\left[\left\{\frac{4}{3} C_{F}^{5}\right\} L_{z}^{8}+\left\{\frac{32}{9} C_{F}^{4} n_{f}\right.\right. \\
& \left.\left.+\left(24-\frac{64}{3} \zeta_{2}\right) C_{F}^{5}+\left(-\frac{272}{9}+\frac{32}{3} \zeta_{2}\right) C_{A} C_{F}^{4}\right\} L_{z}^{7}+\mathcal{O}\left(L_{z}^{6}\right)\right]+\boldsymbol{a}_{\boldsymbol{s}}^{\mathbf{6}}\left[\left\{\frac{8}{15} C_{F}^{6}\right\} L_{z}^{10}\right. \\
& \left.+\left\{\frac{56}{27} C_{F}^{5} n_{f}+\left(12-\frac{32}{3} \zeta_{2}\right) C_{F}^{6}+\left(-\frac{452}{27}+\frac{16}{3} \zeta_{2}\right) C_{A} C_{F}^{5}\right\} L_{z}^{9}+\mathcal{O}\left(L_{z}^{8}\right)\right] \\
& +\boldsymbol{a}_{\boldsymbol{s}}^{7}\left[\left\{\frac{8}{45} C_{F}^{7}\right\} L_{z}^{12}+\left\{\frac{128}{135} C_{F}^{6} n_{f}+\left(\frac{24}{5}-\frac{64}{15} \zeta_{2}\right) C_{F}^{7}-\left(\frac{992}{135}\right.\right.\right. \\
& \left.\left.\left.-\frac{32}{15} \zeta_{2}\right) C_{A} C_{F}^{6}\right\} L_{z}^{11}+\mathcal{O}\left(L_{z}^{10}\right)\right] .
\end{aligned}
$$

In addition, for the gluon initiated process, we predict

$$
\begin{aligned}
\Delta_{g}^{N S V}= & a_{s} \Delta_{g}^{N S V(1)}+a_{s}^{2} \Delta_{g}^{N S V(2)}+a_{s}^{3} \Delta_{g}^{N S V(3)}+\boldsymbol{a}_{\boldsymbol{s}}^{4}\left[\left\{-\frac{16}{3} C_{A}^{4}\right\} L_{z}^{7}-\left\{\frac{32}{3} C_{A}^{3} n_{f}\right.\right. \\
& \left.-104 C_{A}^{4}\right\} L_{z}^{6}+\left\{\frac{5132}{27} C_{A}^{3} n_{f}-\frac{176}{27} C_{A}^{2} n_{f}^{2}+\left(-\frac{8072}{9}+176 \zeta_{2}\right) C_{A}^{4}\right\} L_{z}^{5} \\
& \left.+\mathcal{O}\left(L_{z}^{4}\right)\right]+\boldsymbol{a}_{\boldsymbol{s}}^{5}\left[\left\{-\frac{8}{3} C_{A}^{5}\right\} L_{z}^{9}+\left\{-\frac{76}{9} C_{A}^{4} n_{f}+\frac{682}{9} C_{A}^{5}\right\} L_{z}^{8}-\left\{\frac{800}{81} C_{A}^{3} n_{f}^{2}\right.\right. \\
& \left.\left.-\frac{18088}{81} C_{A}^{4} n_{f}-\left(\frac{78488}{81}-\frac{448}{3} \zeta_{2}\right) C_{A}^{5}\right\} L_{z}^{7}+\mathcal{O}\left(L_{z}^{6}\right)\right]+\boldsymbol{a}_{s}^{6}\left[-\left\{\frac{16}{15} C_{A}^{6}\right\} L_{z}^{11}\right. \\
& -\left\{\frac{656}{135} C_{A}^{5} n_{f}-\frac{5552}{135} C_{A}^{6}\right\} L_{z}^{10}+\left\{-\frac{80}{9} C_{A}^{4} n_{f}^{2}+\frac{14252}{81} C_{A}^{5} n_{f}-\left(\frac{58496}{81}\right.\right. \\
& \left.\left.\left.-\frac{272}{3} \zeta_{2}\right) C_{A}^{6}\right\} L_{z}^{9}+\mathcal{O}\left(L_{z}^{8}\right)\right]+\boldsymbol{a}_{\boldsymbol{s}}^{7}\left[-\left\{\frac{16}{45} C_{A}^{7}\right\} L_{z}^{13}-\left\{\frac{296}{135} C_{A}^{6} n_{f}\right.\right. \\
& \left.-\frac{2396}{135} C_{A}^{7}\right\} L_{z}^{12}+\left\{-\frac{256}{45} C_{A}^{5} n_{f}^{2}+\frac{41864}{405} C_{A}^{6} n_{f}-\left(\frac{164912}{405}-\frac{128}{3} \zeta_{2}\right) C_{A}^{7}\right\} L_{z}^{11} \\
& \left.+\mathcal{O}\left(L_{z}^{10}\right)\right] .
\end{aligned}
$$

Our predictions for $\log ^{7}(1-z), \log ^{6}(1-z)$ and $\log ^{5}(1-z)$ terms at fourth order for $\Delta_{q^{S}}$ agree with that of $[3,4]$.

In summary, if we know $\Psi_{J}^{c}$ up to $n$th order, we can predict $\left(\mathcal{D}_{2 i-2 n+1}, \mathcal{D}_{2 i-2 n}\right)$ and $\log ^{2 i-n}(1-z)$ at every order in $a_{s}^{i}$ for all $i$, see table 1 . We present the general structure of the NSV partonic CFs $\Delta_{c}^{N S V}$ to fourth order in $a_{s}$ in appendix A and in the supplementary material file attached to this paper. 
The fact that the master formula has the predictive nature to all orders in $a_{s}$ in terms of distributions and $\log (1-z)$ terms in $\Delta_{c}$ can be exploited to resum them to all orders. This will be discussed in the next section.

\section{Resummation in $N$ space}

In the last section, we developed a formalism in $z$ space to study SV and NSV contributions to DIS and SIA processes. Our all order result for $\Psi_{J}^{c}$ can predict tower of SV logarithms for $\Delta_{c}$ through the SV distributions $\mathcal{D}_{j}$ and NSV $\operatorname{logarithms~} \log ^{j}(1-z), j=0,1, \cdots$ at every order in $a_{s}$. This is possible because the knowledge of SV and NSV terms for $\Delta_{c}$ up to a given order $m$ in $a_{s}$, say $a_{s}^{i}, i=0, \cdots, m$, contains valuable information through $\Psi_{J}^{c}$, for LL, NLL etc at every order in $a_{s}^{j}$ with $j>m$, i.e., for terms at $a_{s}^{j}, j=m, \cdots, \infty$. The reason for this is due to the fact that the constituents of $\Psi_{J}^{c}$, namely the FF, $Z_{U V, c}, \Phi_{J}^{c}$ and AP kernels, satisfy differential equations and their perturbative solutions have all order predictions for certain logarithms, such as logarithms of $Q^{2}, \mu_{R}^{2}, \mu_{F}^{2}$ and also logarithms of the form $\log ^{j}(1-z) /(1-z)^{k}, j=0,1, \cdots, \infty, k=0,1$. The structure of these logarithms is controlled by UV and IR anomalous dimensions. Presence of such logarithms is a unique feature of any perturbative expansions and is considered advantageous to evaluate whether the perturbative series is reliable or not. They can also become large at every order posing problem for the perturbative series. For example, the distributions $\mathcal{D}_{j}$ and the NSV terms $\log ^{j}(1-z)$ in threshold region can become large at every order in $a_{s}$. In practice, there are situations when the order of perturbation increases, the contributions from distributions $\mathcal{D}_{j}$ also increase such that the product $a_{s}^{j} \mathcal{D}_{j-1}$ is of order one at every order $j=1, \cdots, \infty$. This means that we need to take into account order one terms to all orders in perturbation theory to make any sensible prediction.

Since we are dealing with distributions in $\Psi_{J}^{c}$ and convolutions in $\Delta_{c}$, it is convenient to work in Mellin space $N$. The distributions in $N$ space are well defined. Also the convolutions in $z$ space become normal products in $N$ space. Hence, it is easy to study the order one terms in $N$ space. The threshold limit namely $z \rightarrow 1$ where the SV distributions and NSV logarithms $\log ^{j}(1-z)$ dominate, corresponds to $N \rightarrow \infty$ in Mellin space. We do not strictly take $N \rightarrow \infty$ as we are interested in NSV terms. In $N$ space, in the large $N$ limit, the Mellin moment of $z$ space SV gives $\log ^{i}(N)$ terms as well as $1 / N^{j} \log ^{i}(N)$ terms. However, the $z$ space NSV terms will always give $1 / N^{j} \log ^{i}(N)$ terms. While performing Mellin moments, we drop $1 / N^{j} \log ^{i}(N)$ terms with $j>1$ for all $i$ at every order in $a_{s}$. This way, we have in $N$ space, SV contributions which contain only $\log ^{i}(N)$ terms whereas NSV contains only $1 / N \log ^{i}(N)$ term. The order one terms that we found in $z$ space in the threshold limit will show up in the Mellin space through $\left(a_{s} \beta_{0} \log (N)\right)^{k}$ with $k>0$. Reorganisation of the perturbative series taking into account these order one terms consistently can be achieved through the procedure called resummation. In the rest of the section, we show how these order one terms in $\Psi_{J}^{c}$ can be summed to all orders in perturbation theory. The resummed results in Mellin space taking into account only SV terms are available in the literature for variety of processes, see [44, 51-62]. In the following, we derive the $\mathrm{N}$ space resummed result for the NSV logarithms. Unlike the resummed SV 
contributions, the NSV terms organise themselves in double series expansion in both $a_{s}$ as well as in $\log (N)$. In addition, we find that the resummed expression for NSV part is $1 / N$ suppressed compared to SV.

It is convenient to use the integral representations of $\Phi_{J, A}^{c}$ and $\Phi_{J, B}^{c}$ given in eq. (2.20) and eq. (2.21) respectively to perform the Mellin moment in the large $N$ limit to obtain SV $\log ^{i}(N)$ terms as well as NSV $1 / N \log ^{i}(N)$ terms. Substituting eq. (2.20) and eq. (2.21) in $\Delta_{c}$, the Mellin moment of eq. (2.8) takes the following form,

$$
\Delta_{c, N}\left(Q^{2}, \mu_{R}^{2}, \mu_{F}^{2}\right)=C_{0}^{c}\left(Q^{2}, \mu_{R}^{2}, \mu_{F}^{2}\right) \exp \left(\Psi_{J, N}^{c}\left(Q^{2}, \mu_{F}^{2}\right)\right)
$$

$\Psi_{J, N}^{c}$ in the above equation is twice the Mellin moment of $\Psi_{J, \mathcal{D}}^{c}$,

$$
\Psi_{J, N}^{c}\left(Q^{2}, \mu_{F}^{2}\right)=2 \int_{0}^{1} d z z^{N-1} \Psi_{J, \mathcal{D}}^{c}\left(Q^{2}, \mu_{F}^{2}, z\right) .
$$

where

$$
\Psi_{J, \mathcal{D}}^{c}\left(Q^{2}, \mu_{F}^{2}, z\right)=\frac{1}{2} \int_{\mu_{F}^{2}}^{Q^{2}(1-z)} \frac{d \lambda^{2}}{\lambda^{2}} P_{c c}^{\prime}\left(a_{s}\left(\lambda^{2}\right), z\right)+\mathcal{Q}^{c}\left(a_{s}\left(Q^{2}(1-z)\right), z\right),
$$

and

$$
\mathcal{Q}^{c}\left(a_{s}\left(Q^{2}(1-z)\right), z\right)=\left(\frac{1}{2(1-z)} \bar{G}_{J, S V}^{c}\left(a_{s}\left(Q^{2}(1-z)\right)\right)\right)_{+}+\bar{\varphi}_{f, c}\left(a_{s}\left(Q^{2}(1-z)\right), z\right) .
$$

The $N$ independent coefficient $C_{0}^{c}$ results from the finite parts of $\mathrm{FF}, \Gamma_{c c}$ and the coefficient of $\delta(1-z)$ of $\Phi_{J}^{c}$. We expand $C_{0}^{c}$ in powers of $a_{s}$ as:

$$
C_{0}^{c}\left(Q^{2}, \mu_{R}^{2}, \mu_{F}^{2}\right)=\sum_{i=0}^{\infty} a_{s}^{i}\left(\mu_{R}^{2}\right) C_{0 i}^{c}\left(Q^{2}, \mu_{R}^{2}, \mu_{F}^{2}\right)
$$

where the coefficients $C_{0 i}^{c}$ are presented in the supplementary material file attached to this paper. Also,the results of $C_{0}^{c}$ for the photon-exchange DIS can be found in [52]. Both $C_{0}^{c}$ and $\Psi_{J, N}^{c}$ in eq. (4.1) depend on process dependent quantities as well as the universal anomalous dimensions, $A^{c}, B^{c}, C^{c}, D^{c}, f^{c}$ and set of SV coefficients $\bar{G}_{J, S V}^{c}$ and NSV coefficients $\bar{\varphi}_{f, c^{*}}$.

Before we proceed to the details of computation, we make few remarks on the $N$ space result that we obtained. There have been several studies on the all order structure of NSV terms, see $[65-72,121,122]$ in order to better understand the underlying IR physics. We find that our result given in eq. (4.1) is similar to the one which was conjectured in [65]. However, we differ from eq. (37) in [65], in the upper limit of the integral, the presence of extra term $\bar{\varphi}_{f, c}$ and the explicit dependence on the variable $z$. While these differences do not disturb the SV predictions, they will give NSV terms different from those obtained using eq. (37) of [65].

Our next task is to perform the Mellin moment of SV and NSV terms, where we keep all terms till $\mathcal{O}(1 / N)$ and drop the rest which have higher power in $1 / \mathrm{N}$. We encounter two types of integrals, namely integrals over distributions $\mathcal{D}_{i}, i=0,1, \cdots$ and those over regular terms $\log ^{i}(1-z), i=0,1, \cdots$. Care is needed to deal with the integrals of distributions. The 
section 2.2 of [65] contain results that are suitable to obtain Mellin moments of distributions as well as regular terms in the large $N$ limit. Following, [65], we replace $\int d z\left(z^{N-1}-1\right) /(1-$ $z)$ and $\int d z z^{N-1}$ by $\int \theta(1-z-1 / N) /(1-z)$ and apply the operators $\Gamma_{A}\left(N \frac{d}{d N}\right)$ and $\Gamma_{B}\left(N \frac{d}{d N}\right)$ on them respectively. The $\Gamma_{A}\left(N \frac{d}{d N}\right)$ and $\Gamma_{B}\left(N \frac{d}{d N}\right)$ are given by

$$
\begin{aligned}
\Gamma_{A}(x) & =\sum_{k=0}-\gamma_{k}^{A} x^{k}, & \Gamma_{B}(x) & =\sum_{k=1} \gamma_{k}^{B} x^{k}, \\
\gamma_{k}^{A} & =\frac{\Gamma_{k}(N)}{k !}(-1)^{k-1}, & \gamma_{k+1}^{B} & =\frac{\Gamma^{(k)}(1)}{k !}(-1)^{k} .
\end{aligned}
$$

and they are listed in the appendix [C] of [74]. Applying these operators, we find

$$
\begin{aligned}
\Psi_{J, N}^{c}= & \int_{Q^{2} / N}^{Q^{2}} \frac{d \lambda^{2}}{\lambda^{2}}\left\{\left(\ln \frac{\lambda^{2} N}{Q^{2}}+\gamma_{1}^{A}\right) A^{c}\left(a_{s}\left(\lambda^{2}\right)\right)-\bar{G}_{J, S V}^{c}\left(a_{s}\left(\lambda^{2}\right)\right)-\frac{1}{N} \xi^{c}\left(a_{s}\left(\lambda^{2}\right), N\right)\right. \\
& \left.-\lambda^{2} \frac{d}{d \lambda^{2}} \mathcal{F}^{c}\left(a_{s}\left(\lambda^{2}\right), N\right)\right\}+\mathcal{F}^{c}\left(a_{s}\left(Q^{2}\right), N\right) \\
& +\int_{\mu_{F}^{2}}^{Q^{2}} \frac{d \lambda^{2}}{\lambda^{2}}\left\{\left(-\gamma_{1}^{A}-\log (N)\right) A^{c}\left(a_{s}\left(\lambda^{2}\right)\right)+\frac{1}{N} \xi^{c}\left(a_{s}\left(\lambda^{2}\right), N\right)\right\},
\end{aligned}
$$

where

$$
\mathcal{F}^{c}\left(a_{s}, N\right)=\mathcal{F}_{A}^{c}\left(a_{s}\right)+\frac{1}{N} \mathcal{F}_{B}^{c}\left(a_{s}, N\right) .
$$

with $\mathcal{F}_{A}^{c}\left(a_{s}\right)$ and $\mathcal{F}_{B}^{c}\left(a_{s}, N\right)$ are found to be

$$
\mathcal{F}_{A}^{c}\left(a_{s}\right)=-\gamma_{1}^{A} \bar{G}_{J, S V}^{c}\left(a_{s}\right)+\sum_{i=0}^{\infty} \gamma_{i+2}^{A}\left(-\beta\left(a_{s}\right) \frac{\partial}{\partial a_{s}}\right)^{i}\left\{A^{c}\left(a_{s}\right)+\beta\left(a_{s}\right) \frac{\partial}{\partial a_{s}} \bar{G}_{J, S V}^{c}\left(a_{s}\right)\right\}
$$

and

$$
\begin{aligned}
\mathcal{F}_{B}^{c}\left(a_{s}, N\right)= & 2 \gamma_{1}^{B} \bar{\varphi}_{f, c}\left(a_{s}, N\right)-2 \gamma_{2}^{B}\left(\lambda^{2} \frac{d}{d \lambda^{2}} \bar{\varphi}_{f, c}\left(a_{s}, N\right)+\frac{1}{2} \tilde{\xi}^{c}\left(a_{s}, N\right)\right) \\
& +2\left(\gamma_{3}^{B}+\tilde{\gamma}^{B}\right)\left(\lambda^{2} \frac{d}{d \lambda^{2}}\left\{\lambda^{2} \frac{d}{d \lambda^{2}} \bar{\varphi}_{f, c}\left(a_{s}, N\right)+\frac{1}{2} \tilde{\xi}^{c}\left(a_{s}, N\right)\right\}+\frac{1}{2} C^{c}\left(a_{s}\right)\right) .
\end{aligned}
$$

In the above equation, $\tilde{\gamma}^{B}=\sum_{i=4}^{\infty} \gamma_{i}^{B}\left(N \frac{d}{d N}\right)^{i-3}$. For brevity we denote $a_{s}\left(\lambda^{2}\right)$ as $a_{s}$ in all the above equations. Also,

$$
\tilde{\xi}^{c}\left(a_{s}, N\right)=D^{c}\left(a_{s}\right)-C^{c}\left(a_{s}\right) \log (N), \quad \xi^{c}\left(a_{s}, N\right)=\gamma_{1}^{B} \tilde{\xi}^{c}\left(a_{s}, N\right)-\gamma_{2}^{B} C^{c}\left(a_{s}\right) .
$$

What remains to be done now is the integration over $\lambda^{2}$ in eq. (4.8) or equivalently over $a_{s}$. Care is needed to keep the order one term, namely terms of the form $\omega^{j}, j=1, \cdots, \infty$, with $\omega=a_{s}\left(\mu_{R}^{2}\right) \beta_{0} \log (N)$, at every order in $a_{s}\left(\mu_{R}^{2}\right)$. If we use RG equation for $a_{s}$ and replace integration over $\lambda^{2}$ by $a_{s}$, we obtain the result that is expected to be a function of $a_{s}\left(Q^{2} / N\right)$ as well as $a_{s}\left(Q^{2}\right)$. Both these $a_{s}$ s can be expanded around $a_{s}\left(\mu_{R}^{2}\right)$ using RG 
of the strong coupling constant keeping order one $\omega$ s intact. Alternatively, we can achieve this by performing the integrations over $\lambda$ by using $a_{s}\left(\lambda^{2}\right)$ given by

$$
\begin{aligned}
a_{s}\left(\lambda^{2}\right)= & \left(\frac{a_{s}\left(\mu_{R}^{2}\right)}{l}\right)\left[1-\frac{a_{s}\left(\mu_{R}^{2}\right)}{l} \frac{\beta_{1}}{\beta_{0}} \log (l)+\left(\frac{a_{s}\left(\mu_{R}^{2}\right)}{l}\right)^{2}\left(\frac { \beta _ { 1 } ^ { 2 } } { \beta _ { 0 } ^ { 2 } } \left(\log ^{2}(l)-\log (l)\right.\right.\right. \\
& \left.+l-1)-\frac{\beta_{2}}{\beta_{0}}(l-1)\right)+\left(\frac{a_{s}\left(\mu_{R}^{2}\right)}{l}\right)^{3}\left(\frac { \beta _ { 1 } ^ { 3 } } { \beta _ { 0 } ^ { 3 } } \left(2(1-l) \log (l)+\frac{5}{2} \log ^{2}(l)\right.\right. \\
& \left.-\log ^{3}(l)-\frac{1}{2}+l-\frac{1}{2} l^{2}\right)+\frac{\beta_{3}}{2 \beta_{0}}\left(1-l^{2}\right)+\frac{\beta_{1} \beta_{2}}{\beta_{0}^{2}}(2 l \log (l) \\
& -3 \log (l)-l(1-l)))]
\end{aligned}
$$

where $l=1-\beta_{0} a_{s}\left(\mu_{R}^{2}\right) \log \left(\mu_{R}^{2} / \lambda^{2}\right)$ and $\beta_{i}$ are the coefficients of QCD beta function $\beta\left(a_{s}\right)=$ $-\sum_{i=0}^{\infty} a_{s}^{i+2} \beta_{i}$ known to five loops, see [123-126]. The latter approach is easier to retain order one $\omega$ s at every order in $a_{s}\left(\mu_{R}^{2}\right)$ and we followed this in our paper. Since $\bar{\varphi}_{f, c}$ depends both on $a_{s}$ and $\log (N)$, we make a double series expansion as follows:

$$
\bar{\varphi}_{f, c}\left(a_{s}, N\right)=\sum_{i=1}^{\infty} \sum_{k=0}^{i} a_{s}^{i} \frac{1}{2} \bar{\varphi}_{c, i}^{(k)}(-\log (N))^{k} .
$$

using the expansion for $\bar{\varphi}_{f, c}$ and expanding other quantities in the exponent in powers of $a_{s}$, and using eq. (4.13) for $a_{s}\left(\lambda^{2}\right)$ in eq. (4.8). We obtain

$$
\begin{aligned}
\Psi_{J, N}^{c}= & \log \left(g_{0}^{c}\left(a_{s}\left(\mu_{R}^{2}\right)\right)\right)+\tilde{g}_{1}^{c}(\omega) \log (N)+\sum_{i=0}^{\infty} a_{s}^{i}\left(\mu_{R}^{2}\right) \tilde{g}_{i+2}^{c}(\omega) \\
& +\frac{1}{N} \sum_{i=0}^{\infty} a_{s}^{i}\left(\mu_{R}^{2}\right) h_{i}^{c}(\omega, N)
\end{aligned}
$$

where $\tilde{g}_{i}^{c}$ and $h_{i}^{c}(\omega, N)$ are defined as,

$$
\begin{gathered}
\tilde{g}_{i}^{c}(\omega)=g_{i}^{c}(\omega)+\frac{1}{N} \bar{g}_{i}^{c}(\omega), \\
h_{0}^{c}(\omega, N)=h_{00}^{c}(\omega)+h_{01}^{c}(\omega) \log (N), \quad h_{i}^{c}(\omega, N)=\sum_{k=0}^{i} h_{i k}^{c}(\omega) \log ^{k}(N) .
\end{gathered}
$$

Here we have dropped terms order $\mathcal{O}\left(1 / N^{i}\right), i>1$. Also, the function $\log \left(g_{0}^{c}\left(a_{s}\right)\right)$ is expanded in powers of $a_{s}$ as,

$$
\log \left(g_{0}^{c}\left(a_{s}\left(\mu_{R}^{2}\right)\right)\right)=\sum_{i=1}^{\infty} a_{s}^{i}\left(\mu_{R}^{2}\right) g_{0, i}^{c} .
$$

In eq. (4.15), the coefficients $g_{0}^{c}$ and $g_{i}^{c}, i=1,2, \cdots$ correspond to SV part, whereas $\bar{g}_{i}^{c}$ and $h_{i}^{c}, i=1,2, \cdots$ correspond to NSV part. The coefficients $g_{i}^{c}(\omega)$ are identical to those in [52] obtained from the resummed formula for SV terms. Note that $g_{i}^{c}(\omega)$ becomes zero in the limit $\omega \rightarrow 1$. The coefficient $g_{0}^{c}\left(a_{s}\right)$ (see [52]) is given in the supplementary material file attached to this paper. The $N$ independent coefficients $C_{0}^{c}$ and $g_{0}^{c}$ can be combined as

$$
\tilde{g}_{0}^{c}\left(Q^{2}, \mu_{R}^{2}, \mu_{F}^{2}\right)=C_{0}^{c}\left(Q^{2}, \mu_{R}^{2}, \mu_{F}^{2}\right) g_{0}^{c}\left(a_{s}\left(\mu_{R}^{2}\right)\right),
$$


and is expanded in terms of $a_{s}\left(\mu_{R}^{2}\right)$ as,

$$
\tilde{g}_{0}^{c}\left(a_{s}\left(\mu_{R}^{2}\right)\right)=\sum_{i=0}^{\infty} a_{s}^{i}\left(\mu_{R}^{2}\right) \tilde{g}_{0, i}^{c}
$$

Here, $\tilde{g}_{0, i}^{c}$ are found to be identical to $g_{0 k}^{D I S}$ given in [52]. While both $z$ space as well as $N$ space results contain same information, the summation of order one $\omega$ terms to all orders in perturbation theory can be conveniently performed only in $N$ space. We find that the functions $g_{i}^{c}, \bar{g}_{i}^{c}$ and $h_{i}^{c}$ sum up $\omega$ to all orders in perturbation theory.

In eq. (4.15), $\bar{g}_{1}^{c}$ is found to be identically zero and the remaining terms $\bar{g}_{i}^{c}, i=2,3, \cdots$ are functions of the cusp anomalous dimension $A^{c}$ and the function $\bar{G}_{J, S V}^{c}$ of eq. (4.4). $\bar{g}_{i}^{c}(\omega)$ contain no explicit $\log (N)$ terms. The functions $h_{i}^{c}$ in eq. (4.15) result from the Mellin moment of $\Phi_{J, B}^{c}$ and hence depend on the anomalous dimensions $C^{c}, D^{c}$ and the function $\bar{\varphi}_{f, c}$. We find that the coefficient $h_{01}^{c}$ is proportional to $C_{1}^{c}$ which is identically zero, and hence at order $a_{s}^{0}$, there is no $(1 / N) \log (N)$ term.

We also observe from the explicit calculations, that there exist certain transformation rule which relates to the resummation coefficients $\tilde{g}_{i}^{c}$ and $h_{i}^{c}$ of DIS with the corresponding coefficients of DY/Higgs production presented in [74]. These rules are found to be,

$$
\begin{gathered}
\tilde{g}_{1}^{D Y}(\omega)=2 \tilde{g}_{1}^{D I S}(2 \omega), \quad \tilde{g}_{i+1}^{D Y}(\omega)=\left.\tilde{g}_{i+1}^{D I S}(2 \omega)\right|_{\left\{B_{i} \rightarrow 0, \gamma_{i}^{A} \rightarrow 2^{i} \gamma_{i}^{A}\right\}}, \quad i>0, \\
h_{i k}^{D Y}(\omega, N)=\left.2^{k} h_{i k}^{D I S}(2 \omega, N)\right|_{\left\{D_{i} \rightarrow 2 D_{i}, \gamma_{i}^{B} \rightarrow 2^{(i-1)} \gamma_{i}^{B}, \bar{\varphi}_{i}^{(k)} \rightarrow 2^{(1-k)} \bar{\varphi}_{i}^{(k)}\right\}} .
\end{gathered}
$$

The reason behind the existence of such transformation rule follows from the fact that $\omega$ is related to $\log N$ in DIS whereas for DY/Higgs it is related to $\log N^{2}$. This dependency resurfaces at every stages of the calculation. The scaling of $\gamma_{i}^{A}\left(\gamma_{i}^{B}\right)$ reflects the same dependency as they are coefficients of the derivative of $\log N$ in the expansion of $\Gamma_{A}\left(\Gamma_{B}\right)$ defined in eq. (4.6). Also $\bar{\varphi}_{i}^{(k)}$, being coefficients of $\log N$ (see eq. (4.14)), gets scaled accordingly. Hence the above relations between DIS and DY/Higgs manifest the aforementioned dependency. The results of the coefficients $g_{i}^{c}(\omega)$ are given in [127] and $\bar{g}_{i}^{c}(\omega)$ and $h_{i k}(\omega, N)$ for DIS up to four loops are given in appendix C, D. For completeness we also provide them in the supplementary material file attached to this paper.

Let us now study the all order structure of the exponent in the $N$ space. First of all, it is clear that working in $N$ space in the large $N$ limit (keeping order $\mathcal{O}(1 / N)$ terms) allows us to cast the entire exponent in a compact form through the functions $g_{i}^{c}, \bar{g}_{i}^{c}$ and $h_{i}^{c}$, each of which is a function of $\omega$. These functions carry all order information of SV and NSV logarithms. This is not surprising because $\Phi_{J}^{c}$ does contain the same information in $z$ space, however, with no compact looking form. Note that in $z$ space we have the inherent scale $Q^{2}(1-z)$ of the process appearing at every order with $\hat{a}_{s}$ through $\hat{a}_{s}^{i}\left(Q^{2}(1-z)\right)^{i \epsilon / 2}$ term. This results in $a_{s}\left(Q^{2}(1-z)\right)$ through UV renormalisation which demonstrates the all order prediction of logarithm through distributions and $\log (1-z)$ when expanded around $a_{s}\left(\mu_{R}^{2}\right)$. In $N$ space, these logarithms can be systematically summed up at every order $a_{s}\left(\mu_{R}^{2}\right)$ through the functions $g_{i}^{c}, \bar{g}_{i}^{c}$ and $h_{i}^{c}$ given in the exponent. We find that unlike SV part, NSV part is a double series expansion in $a_{s}\left(\mu_{R}^{2}\right)$ and $\log (N)$. The explicit $\log (N) \mathrm{s}$ 
in the NSV functions $h_{i}^{c}$ comes from explicit $\log (1-z)$ terms in the expansion of $\bar{\varphi}_{f, c}$ and these logarithms do not demonstrate any all order structure and hence can not be summed like the ones appear in the argument of $a_{s}$. Note that the index $i$ in the functions $g_{i}^{c}$ etc that appears in the exponent determine the accuracy of SV and NSV logarithms that are summed up to all orders. Hence, expanding the $\Phi_{J, A}^{c}$ or its Mellin moment around $a_{s}\left(\mu_{R}^{2}\right)$ allows us to make definite predictions for SV and NSV logarithms with given accuracy to all orders in $a_{s}\left(\mu_{R}^{2}\right)$. For example if we have $\Phi_{J, A}^{c}$ up to order $a_{s}$, we can predict terms of the form $a_{s}^{i} \mathcal{D}_{2 i-1}(z)$ in $\Phi_{J, A}^{c}$ for all $i>1$. The functions $\tilde{g}_{0,0}$ and $g_{1}$ in the exponent can predict leading $a_{s}^{i} \log ^{2 i}(N)$ terms for all $i>1$. If we include $\tilde{g}_{0,1}$ and $g_{2}$ terms, then we can predict next to leading $a_{s}^{i} \log ^{2 i-1}(N)$ terms for all $i>2$. In general, the resummed result with terms $\tilde{g}_{0,0}^{c}, \cdots \tilde{g}_{0, n-1}^{c}$ and $g_{1}^{n}, \cdots, g_{n}^{c}$ can predict $a_{s}^{i} \log ^{2 i-n+1}(N)$. The inclusion of sub leading

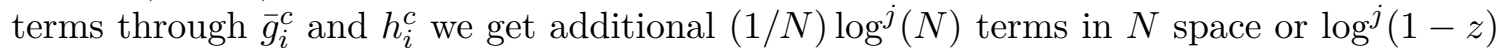
terms in $z$ space. In perturbative QCD, $C_{1}^{c}=0$, where $c=q, \bar{q}, g$. Like the SV part of the resummed exponent, the $1 / N$ suppressed terms also organises themselves by keeping $\omega=a_{s}\left(\mu_{R}^{2}\right) \beta_{0} \log (N)$ terms as order one at every order in $a_{s}\left(\mu_{R}^{2}\right)$. In addition, we find that at a given order $a_{s}^{i}\left(\mu_{R}^{2}\right)$, the $1 / N$ coefficient is a polynomial in $\log (N)$ with the order $i$. Again, we find that using $\tilde{g}_{0,0}^{c}, g_{1}^{c}, g_{2}^{c}$ and $\bar{g}_{1}^{c}, \bar{g}_{2}^{c}, h_{0}^{c}, h_{1}^{c}$, one can predict $\left(a_{s}^{i} / N\right) \log ^{2 i-1}(N)$ terms for all $i>1$. Similarly, along with the previous ones, $\tilde{g}_{0,1}^{c}, g_{3}^{c}$ and $\bar{g}_{3}^{c}, h_{2}^{c}$, one can predict $\left(a_{s}^{i} / N\right) \log ^{2 i-2}(N)$ for all $i>2$. This way, the resummed result with $\bar{g}_{2}^{c}, \cdots, \bar{g}_{n+1}^{c}$ and $h_{1}^{c}, \cdots, h_{n}^{c}$ along with $\tilde{g}_{0,0}, \cdots, \tilde{g}_{0, n-1}$ and $g_{1}, \cdots, g_{n+1}$ can predict $\left(a_{s}^{i} / N\right) \log ^{2 i-n}(N)$ for all $i>n$ in Mellin space $N$. To illustrate the above discussion, we compare the three loop predictions obtained from $\tilde{g}_{0, i-1}, g_{i+1}^{c}, \bar{g}_{i+1}^{c}$ and $h_{i}^{c}$ for $i<3$ against the exact three loop results in table 2 . It can be seen that, given the previous order results, all the higher logarithmic coefficients can be exactly predicted. However, lower order $1 / N \log ^{k}(N), k<4$ can not be predicted from our all order result as they require $\bar{\varphi}_{c, 3}^{(k)}$ for $k<4$ which can only be determined from the third order result for CFs. Interestingly, even without the knowledge of these terms, our predictions for $1 / N \log ^{3}(N)$ terms agree with the exact result for several color factors, see table 2. Note that the limitations in the predictions for higher orders from the previous order for NSV terms are in close resemblance with those of higher order predictions for SV terms in $\mathrm{CF}$, given lower order SV exponents. In summary, our all order result, both in $z$ space and $N$ space demonstrates all order structure as well as predictions that have identical features for both SV and NSV terms. The only difference between SV and NSV terms in the exponent is the way they depend on the process. That is, we find that NSV exponents are process dependent unlike SV ones. Table [2] of [74] summarizes these observations at any given order.

\section{$5 \quad$ Physical evolution kernel}

So far, we studied the structure of CFs in the threshold limit taking into account both the dominant SV and sub dominant NSV terms. Recall that we work in a dimensionally regularised quantum field theory where all the divergences are regularised in $4+\epsilon$ dimensions and use modified minimal subtraction scheme to perform both UV renormalisation as well as mass factorisation. Hence, the CFs and PDF or PFF depend on this scheme, 


\begin{tabular}{|l||c|c||c|c||c|c|}
\hline & \multicolumn{2}{|c||}{$\frac{\log ^{5}(N)}{N}$} & \multicolumn{2}{|c|}{$\frac{\log ^{4}(N)}{N}$} & \multicolumn{2}{c|}{$\frac{\log ^{3}(N)}{N}$} \\
\hline \hline$C_{F}^{3}$ & $\frac{256}{27}$ & $\frac{256}{27}$ & $\frac{6499}{40}$ & $\frac{6499}{40}$ & $\frac{167031}{500}+\frac{3584}{27} \zeta_{2}$ & $\frac{167031}{500}+\frac{3584}{27} \zeta_{2}$ \\
$C_{F}^{2} n_{f}$ & 0 & 0 & $-\frac{1600}{81}$ & $-\frac{1600}{81}$ & $-\frac{431451}{1000}$ & $-\frac{105229}{250}+\chi_{1}$ \\
$C_{A} C_{F}^{2}$ & 0 & 0 & $\frac{1760}{27}$ & $\frac{1760}{27}$ & $\frac{38617}{25}-256 \zeta_{2}$ & $\frac{150991}{100}-256 \zeta_{2}+$ \\
& & & & & & $\chi_{2}$ \\
$C_{F} n_{f}^{2}$ & 0 & 0 & 0 & 0 & $\frac{800}{81}$ & $\frac{800}{81}$ \\
$C_{A} C_{F} n_{f}$ & 0 & 0 & 0 & 0 & $-\frac{1760}{27}$ & $-\frac{1760}{27}$ \\
$C_{A}^{2} C_{F}$ & 0 & 0 & 0 & 0 & $\frac{968}{9}$ & $\frac{968}{9}$ \\
\hline
\end{tabular}

Table 2. Comparison of 3-loop resummed predictions against exact results for the DIS structure function $F_{2}$. For each color structure, the left column stands for the exact results and the right column stands for the resummed predictions. The constant $\chi_{1}$ and $\chi_{2}$ depends on $\bar{\varphi}_{q, 3}^{(3)}$.

hence they are unphysical. However, the physical observables comprising of them are blind to this. This means that if we make scheme transformations on CFs and on PDFs or PFF simultaneously, the physical observables are invariant. For example, following [128], we consider an observable $\mathcal{O}\left(Q^{2}\right)=\mathcal{C}_{\mathcal{O}}\left(Q^{2}, \mu_{F}^{2}\right) \mathcal{F}\left(\mu_{F}^{2}\right)$, where $\mathcal{O}$ represent any of the structure functions that appear in the DIS or in the cross section for SIA. The functions $\mathcal{C}_{\mathcal{O}}$ and $\mathcal{F}$ are the corresponding CFs and PDFs or PFFs respectively. They are independently scheme dependent quantities. That is, if we make scheme transformations, namely $\mathcal{C}_{\mathcal{O}} \rightarrow Z \mathcal{C}_{\mathcal{O}}$ and $\mathcal{F} \rightarrow \mathcal{F} / Z$, then $\mathcal{O}$ remains invariant. This fact allows us to construct perturbative quantities out of CFs and PDF/PFF such that they are invariant under scheme transformation. One such quantity is $\mathcal{K}_{\mathcal{O}}$, called physical evolution kernel (PEK) $[79,129]$ and is defined through

$$
Q^{2} \frac{d}{d Q^{2}} \mathcal{O}\left(Q^{2}\right)=\mathcal{K}_{\mathcal{O}}\left(Q^{2}\right) \mathcal{O}\left(Q^{2}\right)
$$

where, $\mathcal{K}_{\mathcal{O}}$ is obtained using the $\mathrm{RG}$ equation for $\mathcal{C}_{\mathcal{O}}$ and is given by

$$
\mathcal{K}_{\mathcal{O}}\left(Q^{2}\right)=\gamma_{\mathcal{O}}\left(Q^{2}\right)+Q^{2} \frac{d C_{\mathcal{O}}\left(Q^{2}\right)}{d Q^{2}} C_{\mathcal{O}}^{-1}\left(Q^{2}\right) .
$$

The anomalous dimension $\gamma_{\mathcal{O}}$ satisfies $\mu_{F}^{2} d / d \mu_{F}^{2}\left(\log \left(\mathcal{F}_{\mathcal{O}}\left(\mu_{F}^{2}\right)\right)=\gamma_{\mathcal{O}}\left(\mu_{F}^{2}\right)\right.$. Being scheme independent, the kernel can be used to understand the perturbative structure of physical quantities. In [128], the crossing relation namely the Drell-Levy-Yan relation [130] between CFs of DIS and of SIA were studied in a scheme invariant way using PEK. In $[64,131]$, next to NSV to DIS was studied using PEK.

A striking observation was made by Moch and Vogt in [63] (and [31, 35]), by studying PEKs of observables in DIS, semi-inclusive $e^{+} e^{-}$annihilation and DY. They showed that the PEKs demonstrate the enhancement of a single-logarithms at large $z$ to all order in $1-z$. Making use of this observation and extending it to all orders in $a_{s}$, the structure of corresponding leading $\log (1-z)$ terms in the kernel can be constrained, which allowed them to predict certain next to SV logarithms at higher orders in $a_{s}$. 
Now that we have an all order results for SV+NSV both in $z$ and $N$ spaces, we can easily predict the structure of leading logarithms in the physical evolution kernel. It is convenient to use the result in $N$ space for this purpose. The PEK for DIS is given by

$$
\mathcal{K}^{c}\left(a_{s}\left(\mu_{R}^{2}\right), N\right)=Q^{2} \frac{d}{d Q^{2}} \log \Delta_{c, N}\left(Q^{2}\right) .
$$

which is invariant under scheme transformation. The kernel $\mathcal{K}^{c}\left(a_{s}\left(\mu_{R}^{2}\right), N\right)$ can be computed order by order in perturbation theory

$$
\mathcal{K}^{c}\left(a_{s}\left(\mu_{R}^{2}\right), N\right)=\sum_{i=1}^{\infty} a_{s}^{i}\left(\mu_{R}^{2}\right) \mathcal{K}_{i-1}^{c}(N)
$$

As in [63], the leading $(1 / N) \log ^{i}(N)$ terms at every order defined by $\overline{\mathcal{K}}^{c}$ :

$$
\overline{\mathcal{K}}_{i}^{c}=\left.\mathcal{K}_{i}^{c}\right|_{(1 / N) \log ^{i}(N)}
$$

can be obtained. Using eq. (4.1), we find that these terms can be obtained directly from the NSV part of $\Psi_{J, N}^{c}$ alone and are given by

$$
\overline{\mathcal{K}}_{i}^{c}=\frac{1}{2} A_{1}^{c} \beta_{0}^{i}+D_{1}^{c} \beta_{0}^{i}-i \beta_{0}^{(i-1)} C_{2}^{c}+i \sum_{j=1}^{i}(-1)^{j+1}\left(\begin{array}{c}
i-1 \\
j-1
\end{array}\right) \beta_{0}^{(i-j+1)} \bar{\varphi}_{c, j}^{(j)} .
$$

The corresponding PEKs for SIA can be obtained from the above DIS PEKs by replacing $D_{1}^{c}, C_{2}^{c}$ and $\bar{\varphi}_{c, i}^{(i)}$ by the respective time-like counter parts. Interestingly at every order in $a_{s}^{i}$ the leading $(1 / N) \log ^{i}(N)$ terms are controlled by $D_{1}^{c}$ and $C_{2}^{c}$ from $L^{c}$ and by $\bar{\varphi}_{c, i}^{(i)}$. This is our prediction for the leading $1 / N$ behavior of the physical evolution kernel, $\mathcal{K}^{c}$, at every order in perturbation theory and is consistent with the observation made by [63] using the known perturbative results.

For photon-exchange DIS and fragmentation functions in SIA we find a complete numerical agreement with [63] up to three loops for all the structure functions upon substituting the known values of $C^{c}, D^{c}$ of space and time-like splitting functions and DIS NSV constants $\bar{\varphi}_{c, i}^{(i)}$ and SIA ones $\tilde{\varphi}_{c, i}^{(i)}$ from eqs. $(2.30),(2.31),(2.33),(2.34)$ respectively. In the following we present the PEKs for the fragmentation functions in SIA, photon-exchange DIS and Higgs-exchange DIS:

$$
\begin{aligned}
& \overline{\mathcal{K}}_{0}^{c}=-2 C_{i} \\
& \overline{\mathcal{K}}_{1}^{c}=-2 \beta_{0} C_{i} \pm 16 C_{i}^{2} \\
& \overline{\mathcal{K}}_{2}^{c}=-2 \beta_{0}^{2} C_{i} \pm 24 \beta_{0} C_{i}^{2} \\
& \overline{\mathcal{K}}_{3}^{c}=-2 \beta_{0}^{3} C_{i} \pm \frac{88}{3} \beta_{0}^{2} C_{i}^{2} \\
& \overline{\mathcal{K}}_{4}^{c}=-2 \beta_{0}^{4} C_{i} \pm \frac{112}{3} \beta_{0}^{3} C_{i}^{2}-4 \beta_{0} \bar{\varphi}_{c, 4}^{(4)} \\
& \overline{\mathcal{K}}_{5}^{c}=-2 \beta_{0}^{5} C_{i} \pm \frac{160}{3} \beta_{0}^{4} C_{i}^{2}-20 \beta_{0}^{2} \bar{\varphi}_{c, 4}^{(4)}+5 \beta_{0} \bar{\varphi}_{c, 5}^{(5)}, \\
& \overline{\mathcal{K}}_{6}^{c}=-2 \beta_{0}^{6} C_{i} \pm \frac{248}{3} \beta_{0}^{5} C_{i}^{2}-60 \beta_{0}^{3} \bar{\varphi}_{c, 4}^{(4)}+30 \beta_{0}^{2} \bar{\varphi}_{c, 5}^{(5)}-6 \beta_{0} \bar{\varphi}_{c, 6}^{(6)} .
\end{aligned}
$$


Here $C_{i}$ is $\left\{C_{A}, C_{F}\right\}$ for $c=\{g, q\}$ respectively. And the plus sign corresponds to SIA and the minus sign is for photon and Higgs-exchange DIS. This owe to the fact that the anomalous dimension $C_{2}$ is equal and opposite in sign for space-like and time-like splitting kernels. Moreover from eq. $(2.31),(2.33),(2.35)$ we can also see that $\bar{\varphi}_{q, k}^{(k)}$ from two loop onwards are equal and opposite in sign giving rise to the above differences in sign between SIA and DIS.

The agreement of our predictions for the leading term in the kernel with those obtained using explicit results for CFs is not surprising because the $\mathrm{K}+\mathrm{G}$ equation that the $\Phi_{J}^{c}$ satisfies and the functions $\bar{G}_{J, L}^{c} \mathrm{~S}$ are similar to physical evolution equation and the PEK respectively. The highest power of $\log (N)$ in the $1 / N$ coefficient of $\overline{\mathcal{K}}^{c}$ is due to the upper limit on the summation in eq. (4.14). We make another interesting comparison between eq. (5.6) and eq. (5.7). The general structure for PEKs in terms of the anomalous dimensions and $\bar{\varphi}_{c, k}^{(k)}$ given in eq. (5.6) shows that $\overline{\mathcal{K}}_{j}^{c}$ at any given order is proportional to $\beta_{0}^{i}, i=j, \cdots, 1$. On the other hand for eq. (5.7) we have coefficients only proportional to $\beta_{0}^{i}$ and $\beta_{0}^{i-1}$ for $\overline{\mathcal{K}}_{i}^{c}$. Hence the substitution of the explicit values of $A_{1}, C_{2}, D_{1}$ and $\bar{\varphi}_{c, k}^{(k)}$ in eq. (5.6) conspires in such a way so as to keep only $\beta_{0}^{i}$ and $\beta_{0}^{i-1}$ at every order, which calls for an explanation. However, this exercise provides a consistency check on our framework. Detailed study on the structure of sub leading contributions to the PEKs, namely, the coefficients of $1 / N \log ^{i-1}(N), 1 / N \log ^{i-2}(N)$ etc at every order $a_{s}^{i}$ can unravel the $\log (N)$ structure of $\bar{\varphi}_{f, c}$. It is to be noted that the results for $\overline{\mathcal{K}}_{4}^{c}, \overline{\mathcal{K}}_{5}^{c}$ and $\overline{\mathcal{K}}_{6}^{c}$ are incomplete due to the unavailability of the full explicit NSV results for the CFs at $a_{s}^{4}, a_{s}^{5}$ and $a_{s}^{6}$ orders.

While the fixed order predictions for CFs of DIS and SIA from our formalism agree with those [63], our formalism provides a result in $z$ that sums up both SV and NSV logarithms to all orders in $a_{s}$ in terms of certain universal anomalous dimensions and process dependent constants. This is possible because we could get an all order solution for the $\mathrm{K}+\mathrm{G}$ equation that results from factorisation and renormalisation group invariance. In addition, our $z$ space result allows to perform resummation of order one terms in the exponent to improve the precision of theoretical predictions from the NSV terms.

\section{Conclusions}

Perturbative structure of the observables in QFT is very rich and the higher order predictions for them provide enormous opportunities to unravel the details of the underlying dynamics. In particular, inclusive observables such as structure functions in DIS and fragmentation functions in SIA can be used to understand factorisation properties in QCD perturbation theory. This requires the knowledge of infrared structure of on-shell amplitudes at higher orders. IR structure of perturbative results show universality through process independent anomalous dimensions, splitting functions, soft distributions and jet functions etc. The universality of these quantities allows us to use the results from one process to make predictions for the other ones. For example, quantities like IR anomalous dimensions, soft functions parametrise resummation exponents in a process independent way allowing us to use them for a class of processes to perform threshold resummation. Often, threshold corrections are important in certain kinematical regions and they contribute 
significantly at every order spoiling the reliability of perturbation theory. Resummation of such contributions to all orders provides a possible solution. The most dominant one, namely SV contributions are resummed, conveniently in Mellin space. They show up as order one contributions through $\omega=a_{s} \beta_{0} \log (N)$ at every order in perturbation theory. Next to SV terms are often not small at every order demanding careful investigation of their structure. Such an investigation would help us to systematically include them to all orders.

In this paper, we begin by studying the structure of $\log ^{k}(1-z), k=0, \cdots, \infty$ NSV terms at every order $a_{s}^{i}$. We used collinear factorisation of inclusive cross sections, their RG invariance and $\mathrm{K}+\mathrm{G}$ equations for the form factors and for the real emission contributions that contribute. We obtain an all order result in $z$ space that describes the IR structure of NSV terms in terms of IR anomalous dimensions $C^{c}$ and $D^{c}$ and the functions $\bar{\varphi}_{f, c}$. Given the result for CF to specific order in $a_{s}$, our $z$ space result can predict SV and NSV terms of CF with certain accuracy to all orders in $a_{s}$. This feature is the result of universal IR structure that these contributions demonstrate.

In addition, we find that the finite part of soft distribution and next to soft distribution functions have integral representations that can be used to study them in Mellin $N$ space. In Mellin $N$ space taking into account $\operatorname{large} \log (N)$ and $1 / N \log (N)$ contributions, one can easily resum order one terms namely $\omega$ to all orders in $a_{s}$. We also find that $\bar{\varphi}_{f, c} \mathrm{~s}$ depend on the hard scattering process breaking the universality that the SV part of the inclusive cross sections enjoy. Like in $z$ space, if we know CFs to certain order in $a_{s}$, our results can predict certain $1 / N$ suppressed $\log (N)$ contributions to all orders in $a_{s}$. While this paper addresses only quark initiated reactions (gluons in Higgs-DIS) both in DIS and SIA, the NSV terms resulting from other scattering reactions can not be ignored and the perturbative structure and possible resummation of them to all orders are need of the hour to obtain any consistent analysis. The resummed result taking into account $1 / N$ corrections will be useful to study the phenomenological importance of NSV contributions to inclusive observables.

\section{Acknowledgments}

We thank Moch for useful discussion, his constant help throughout this project and also for providing third order results for the inclusive reactions.

\section{A NSV Partonic coefficient functions $\Delta_{c}^{N S V}$}

The partonic coefficient function given in eq. (3.1) can be written as,

$$
\Delta_{c}^{(i)}\left(Q^{2}, \mu_{R}^{2}, \mu_{F}^{2}, z\right)=\Delta_{c}^{S V,(i)}\left(Q^{2}, \mu_{R}^{2}, \mu_{F}^{2}, z\right)+\Delta_{c}^{N S V,(i)}\left(Q^{2}, \mu_{R}^{2}, \mu_{F}^{2}, z\right)
$$

where $\Delta_{c}^{S V,(i)}\left(Q^{2}, \mu_{R}^{2}, \mu_{F}^{2}, z\right)$ can be found in $[28,29,33,43,45]$. The $\Delta_{c}^{N S V,(i)}$ to fourth order for DIS and SIA after setting $\mu_{R}^{2}=\mu_{F}^{2}=Q^{2}$ has the following expansion:

$$
\Delta_{c}^{N S V,(i)}(z)=\sum_{k=0}^{(2 i-1)} \Delta_{c}^{i k} \log ^{k}(1-z)
$$


Below, we present the $\Delta_{c}^{i k}$ for DIS( $c=q$ for photon exchange and $c=g$ for Higgs exchange).

$$
\begin{aligned}
& \Delta_{c}^{10}=\bar{\varphi}_{c, 1}^{(0)} \text {, } \\
& \Delta_{c}^{11}=\bar{\varphi}_{c, 1}^{(1)}+D_{1}^{c} \text {, } \\
& \Delta_{c}^{12}=C_{1}^{c} \text {, } \\
& \Delta_{c}^{20}=\bar{\varphi}_{c, 2}^{(0)}+\bar{\varphi}_{c, 1}^{(1)} \zeta_{2}\left(f_{1}^{c}+B_{1}^{c}\right)+2 \bar{\varphi}_{c, 1}^{(0)}\left(\overline{\mathcal{G}}_{S V, 1}^{c}+g_{1}^{c, 1}\right)+D_{1}^{c} \zeta_{2}\left(f_{1}^{c}+B_{1}^{c}\right)-2 C_{1}^{c} \zeta_{3}\left(f_{1}^{c}+B_{1}^{c}\right) \\
& +A_{1}^{c} \bar{\varphi}_{c, 1}^{(1)} \zeta_{3}+A_{1}^{c} D_{1}^{c} \zeta_{3}-\frac{1}{5} A_{1}^{c} C_{1}^{c} \zeta_{2}^{2}-\frac{1}{2}\left(A_{1}^{c}\right)^{2} \zeta_{2}+\frac{1}{2}\left(f_{1}^{c}\right)^{2}+B_{1}^{c} f_{1}^{c}+\frac{1}{2}\left(B_{1}^{c}\right)^{2}, \\
& \Delta_{c}^{21}=\bar{\varphi}_{c, 2}^{(1)}+2 \bar{\varphi}_{c, 1}^{(1)}\left(\overline{\mathcal{G}}_{S V, 1}^{c}+g_{1}^{c, 1}\right)-\bar{\varphi}_{c, 1}^{(0)}\left(\beta_{0}+f_{1}^{c}+B_{1}^{c}\right)+D_{2}^{c}+2 D_{1}^{c}\left(\overline{\mathcal{G}}_{S V, 1}^{c}+g_{1}^{c, 1}\right) \\
& +2 C_{1}^{c} \zeta_{2}\left(f_{1}^{c}+B_{1}^{c}\right)-A_{1}^{c}\left(f_{1}^{c}+B_{1}^{c}\right)-A_{1}^{c} \bar{\varphi}_{c, 1}^{(1)} \zeta_{2}-A_{1}^{c} D_{1}^{c} \zeta_{2}+4 A_{1}^{c} C_{1}^{c} \zeta_{3}, \\
& \Delta_{c}^{22}=\bar{\varphi}_{c, 2}^{(2)}-\bar{\varphi}_{c, 1}^{(1)}\left(\beta_{0}+f_{1}^{c}+B_{1}^{c}\right)-\frac{1}{2} D_{1}^{c}\left(\beta_{0}+2 f_{1}^{c}+2 B_{1}^{c}\right)+C_{2}^{c}+2 C_{1}^{c}\left(\overline{\mathcal{G}}_{S V, 1}^{c}+g_{1}^{c, 1}\right)+\frac{1}{2} A_{1}^{c} \bar{\varphi}_{c, 1}^{(0)} \\
& -2 A_{1}^{c} C_{1}^{c} \zeta_{2}+\frac{1}{2}\left(A_{1}^{c}\right)^{2} \\
& \Delta_{c}^{23}=-\frac{1}{2} C_{1}^{c}\left(\beta_{0}+2 f_{1}^{c}+2 B_{1}^{c}\right)+\frac{1}{2} A_{1}^{c} \bar{\varphi}_{c, 1}^{(1)}+\frac{1}{2} A_{1}^{c} D_{1}^{c}, \\
& \Delta_{c}^{24}=\frac{1}{2} A_{1}^{c} C_{1}^{c}, \\
& \Delta_{c}^{33}=\bar{\varphi}_{c, 3}^{(3)}-\bar{\varphi}_{c, 2}^{(2)}\left(2 \beta_{0}+f_{1}^{c}+B_{1}^{c}\right)+\frac{1}{2} \bar{\varphi}_{c, 1}^{(1)}\left(2 \beta_{0}^{2}+3 f_{1}^{c} \beta_{0}+\left(f_{1}^{c}\right)^{2}+3 B_{1}^{c} \beta_{0}+2 B_{1}^{c} f_{1}^{c}+\left(B_{1}^{c}\right)^{2}\right) \\
& +\frac{1}{6} D_{1}^{c}\left(2 \beta_{0}^{2}+6 f_{1}^{c} \beta_{0}+3\left(f_{1}^{c}\right)^{2}+6 B_{1}^{c} \beta_{0}+6 B_{1}^{c} f_{1}^{c}+3\left(B_{1}^{c}\right)^{2}\right)-C_{2}^{c}\left(\beta_{0}+f_{1}^{c}+B_{1}^{c}\right) \\
& -\frac{1}{2} C_{1}^{c}\left(\beta_{1}+6 \overline{\mathcal{G}}_{S V, 1}^{c} \beta_{0}+2 g_{1}^{c, 1} \beta_{0}+2 f_{2}^{c}+4 f_{1}^{c} \overline{\mathcal{G}}_{S V, 1}^{c}+4 f_{1}^{c} g_{1}^{c, 1}+2 B_{2}^{c}+4 B_{1}^{c} \overline{\mathcal{G}}_{S V, 1}^{c}+4 B_{1}^{c} g_{1}^{c, 1}\right) \\
& +\frac{1}{2} A_{2}^{c} \bar{\varphi}_{c, 1}^{(1)}+\frac{1}{2} A_{2}^{c} D_{1}^{c}+\frac{1}{2} A_{1}^{c} \bar{\varphi}_{c, 2}^{(1)}+A_{1}^{c} \bar{\varphi}_{c, 1}^{(1)}\left(\overline{\mathcal{G}}_{S V, 1}^{c}+g_{1}^{c, 1}\right)-\frac{1}{6} A_{1}^{c} \bar{\varphi}_{c, 1}^{(0)}\left(4 \beta_{0}+3 f_{1}^{c}+3 B_{1}^{c}\right) \\
& +\frac{1}{2} A_{1}^{c} D_{2}^{c}+A_{1}^{c} D_{1}^{c}\left(\overline{\mathcal{G}}_{S V, 1}^{c}+g_{1}^{c, 1}\right)+\frac{1}{2} A_{1}^{c} C_{1}^{c} \zeta_{2}\left(5 \beta_{0}+8 f_{1}^{c}+8 B_{1}^{c}\right) \\
& -\frac{1}{2}\left(A_{1}^{c}\right)^{2}\left(\beta_{0}+2 f_{1}^{c}+2 B_{1}^{c}\right)-\left(A_{1}^{c}\right)^{2} \bar{\varphi}_{c, 1}^{(1)} \zeta_{2}-\left(A_{1}^{c}\right)^{2} D_{1}^{c} \zeta_{2}+5\left(A_{1}^{c}\right)^{2} C_{1}^{c} \zeta_{3}, \\
& \Delta_{c}^{34}=\frac{1}{6} C_{1}^{c}\left(2 \beta_{0}^{2}+6 f_{1}^{c} \beta_{0}+3\left(f_{1}^{c}\right)^{2}+6 B_{1}^{c} \beta_{0}+6 B_{1}^{c} f_{1}^{c}+3\left(B_{1}^{c}\right)^{2}\right)+\frac{1}{2} A_{2}^{c} C_{1}^{c}+\frac{1}{2} A_{1}^{c} \bar{\varphi}_{c, 2}^{(2)} \\
& -\frac{1}{6} A_{1}^{c} \bar{\varphi}_{c, 1}^{(1)}\left(4 \beta_{0}+3 f_{1}^{c}+3 B_{1}^{c}\right)-\frac{1}{12} A_{1}^{c} D_{1}^{c}\left(5 \beta_{0}+6 f_{1}^{c}+6 B_{1}^{c}\right)+\frac{1}{2} A_{1}^{c} C_{2}^{c} \\
& +A_{1}^{c} C_{1}^{c}\left(\overline{\mathcal{G}}_{S V, 1}^{c}+g_{1}^{c, 1}\right)+\frac{1}{8}\left(A_{1}^{c}\right)^{2} \bar{\varphi}_{c, 1}^{(0)}-\frac{3}{2}\left(A_{1}^{c}\right)^{2} C_{1}^{c} \zeta_{2}+\frac{1}{4}\left(A_{1}^{c}\right)^{3}, \\
& \Delta_{c}^{35}=-\frac{1}{12} A_{1}^{c} C_{1}^{c}\left(5 \beta_{0}+6 f_{1}^{c}+6 B_{1}^{c}\right)+\frac{1}{8}\left(A_{1}^{c}\right)^{2} \bar{\varphi}_{c, 1}^{(1)}+\frac{1}{8}\left(A_{1}^{c}\right)^{2} D_{1}^{c}, \\
& \Delta_{c}^{36}=\frac{1}{8}\left(A_{1}^{c}\right)^{2} C_{1}^{c},
\end{aligned}
$$




$$
\begin{aligned}
& \Delta_{c}^{45}=-\frac{1}{12} C_{1}^{c}\left(3 \beta_{0}^{3}+11 f_{1}^{c} \beta_{0}^{2}+9\left(f_{1}^{c}\right)^{2} \beta_{0}+2\left(f_{1}^{c}\right)^{3}+11 B_{1}^{c} \beta_{0}^{2}+18 B_{1}^{c} f_{1}^{c} \beta_{0}+6 B_{1}^{c}\left(f_{1}^{c}\right)^{2}\right. \\
& \left.+9\left(B_{1}^{c}\right)^{2} \beta_{0}+6\left(B_{1}^{c}\right)^{2} f_{1}^{c}+2\left(B_{1}^{c}\right)^{3}\right)-\frac{1}{12} A_{2}^{c} C_{1}^{c}\left(7 \beta_{0}+6 f_{1}^{c}+6 B_{1}^{c}\right)+\frac{1}{2} A_{1}^{c} \bar{\varphi}_{c, 3}^{(3)} \\
& -\frac{1}{6} A_{1}^{c} \bar{\varphi}_{c, 2}^{(2)}\left(7 \beta_{0}+3 f_{1}^{c}+3 B_{1}^{c}\right) \\
& +\frac{1}{12} A_{1}^{c} \bar{\varphi}_{c, 1}^{(1)}\left(9 \beta_{0}^{2}+11 f_{1}^{c} \beta_{0}+3\left(f_{1}^{c}\right)^{2}+11 B_{1}^{c} \beta_{0}+6 B_{1}^{c} f_{1}^{c}+3\left(B_{1}^{c}\right)^{2}\right) \\
& +\frac{1}{12} A_{1}^{c} D_{1}^{c}\left(4 \beta_{0}^{2}+8 f_{1}^{c} \beta_{0}+3\left(f_{1}^{c}\right)^{2}+8 B_{1}^{c} \beta_{0}+6 B_{1}^{c} f_{1}^{c}+3\left(B_{1}^{c}\right)^{2}\right) \\
& -\frac{1}{6} A_{1}^{c} C_{2}^{c}\left(4 \beta_{0}+3 f_{1}^{c}+3 B_{1}^{c}\right) \\
& -\frac{1}{12} A_{1}^{c} C_{1}^{c}\left(5 \beta_{1}+22 \overline{\mathcal{G}}_{S V, 1}^{c} \beta_{0}+10 g_{1}^{c, 1} \beta_{0}+6 f_{2}^{c}+12 f_{1}^{c} \overline{\mathcal{G}}_{S V, 1}^{c}+12 f_{1}^{c} g_{1}^{c, 1}+6 B_{2}^{c}+12 B_{1}^{c} \overline{\mathcal{G}}_{S V, 1}^{c}\right. \\
& \left.+12 B_{1}^{c} g_{1}^{c, 1}\right)+\frac{1}{4} A_{1}^{c} A_{2}^{c} \bar{\varphi}_{c, 1}^{(1)}+\frac{1}{4} A_{1}^{c} A_{2}^{c} D_{1}^{c}+\frac{1}{8}\left(A_{1}^{c}\right)^{2} \bar{\varphi}_{c, 2}^{(1)}+\frac{1}{4}\left(A_{1}^{c}\right)^{2} \bar{\varphi}_{c, 1}^{(1)}\left(\overline{\mathcal{G}}_{S V, 1}^{c}+g_{1}^{c, 1}\right) \\
& -\frac{1}{24}\left(A_{1}^{c}\right)^{2} \bar{\varphi}_{c, 1}^{(0)}\left(5 \beta_{0}+3 f_{1}^{c}+3 B_{1}^{c}\right)+\frac{1}{8}\left(A_{1}^{c}\right)^{2} D_{2}^{c}+\frac{1}{4}\left(A_{1}^{c}\right)^{2} D_{1}^{c}\left(\overline{\mathcal{G}}_{S V, 1}^{c}+g_{1}^{c, 1}\right) \\
& +\frac{1}{12}\left(A_{1}^{c}\right)^{2} C_{1}^{c} \zeta_{2}\left(28 \beta_{0}+27 f_{1}^{c}+27 B_{1}^{c}\right)-\frac{1}{24}\left(A_{1}^{c}\right)^{3}\left(8 \beta_{0}+9 f_{1}^{c}+9 B_{1}^{c}\right)-\frac{3}{8}\left(A_{1}^{c}\right)^{3} \bar{\varphi}_{c, 1}^{(1)} \zeta_{2} \\
& -\frac{3}{8}\left(A_{1}^{c}\right)^{3} D_{1}^{c} \zeta_{2}+\frac{7}{3}\left(A_{1}^{c}\right)^{3} C_{1}^{c} \zeta_{3} \\
& \Delta_{c}^{46}=\frac{1}{12} A_{1}^{c} C_{1}^{c}\left(4 \beta_{0}^{2}+8 f_{1}^{c} \beta_{0}+3\left(f_{1}^{c}\right)^{2}+8 B_{1}^{c} \beta_{0}+6 B_{1}^{c} f_{1}^{c}+3\left(B_{1}^{c}\right)^{2}\right)+\frac{1}{4} A_{1}^{c} A_{2}^{c} C_{1}^{c}+\frac{1}{8}\left(A_{1}^{c}\right)^{2} \bar{\varphi}_{c, 2}^{(2)} \\
& -\frac{1}{24}\left(A_{1}^{c}\right)^{2} \bar{\varphi}_{c, 1}^{(1)}\left(5 \beta_{0}+3 f_{1}^{c}+3 B_{1}^{c}\right)-\frac{1}{48}\left(A_{1}^{c}\right)^{2} D_{1}^{c}\left(7 \beta_{0}+6 f_{1}^{c}+6 B_{1}^{c}\right)+\frac{1}{8}\left(A_{1}^{c}\right)^{2} C_{2}^{c} \\
& +\frac{1}{4}\left(A_{1}^{c}\right)^{2} C_{1}^{c}\left(\overline{\mathcal{G}}_{S V, 1}^{c}+g_{1}^{c, 1}\right)+\frac{1}{48}\left(A_{1}^{c}\right)^{3} \bar{\varphi}_{c, 1}^{(0)}-\frac{1}{2}\left(A_{1}^{c}\right)^{3} C_{1}^{c} \zeta_{2}+\frac{1}{16}\left(A_{1}^{c}\right)^{4}, \\
& \Delta_{c}^{47}=-\frac{1}{48}\left(A_{1}^{c}\right)^{2} C_{1}^{c}\left(7 \beta_{0}+6 f_{1}^{c}+6 B_{1}^{c}\right)+\frac{1}{48}\left(A_{1}^{c}\right)^{3} \bar{\varphi}_{c, 1}^{(1)}+\frac{1}{48}\left(A_{1}^{c}\right)^{3} D_{1}^{c}, \\
& \Delta_{c}^{48}=\frac{1}{48}\left(A_{1}^{c}\right)^{3} C_{1}^{c} .
\end{aligned}
$$

We have put $\Delta_{c}^{30}, \cdots, \Delta_{c}^{32}$ and $\Delta_{c}^{40}, \cdots, \Delta_{c}^{44}$ along with the above results in the supplementary material file as they are lengthy. Next, we present the $\tilde{\Delta}_{c}^{i k}$ for SIA in terms of $\Delta_{c}^{i k}$ for DIS. The expansion coefficients $\bar{\varphi}_{c, i}^{(k)}$ should be replaced with $\tilde{\varphi}_{c, i}^{(k)}$ given in eq. (2.33) and eq. (2.34), in the relations provided below.

$$
\begin{aligned}
& \tilde{\Delta}_{c}^{20}=\Delta_{c}^{20}+3 A_{1}^{c} \zeta_{2} \bar{\varphi}_{c, 1}^{(0)}, \\
& \tilde{\Delta}_{c}^{21}=\Delta_{c}^{21}+3 A_{1}^{c} \zeta_{2}\left(D_{1}^{c}+\bar{\varphi}_{c, 1}^{(1)}\right), \\
& \tilde{\Delta}_{c}^{22}=\Delta_{c}^{22}+3 A_{1}^{c} \zeta_{2} C_{1}^{c}, \\
& \tilde{\Delta}_{c}^{33}=\Delta_{c}^{33}+\frac{3}{2} A_{1}^{c} \zeta_{2}\left(-C_{1}^{c} \beta_{0}-2 f_{1}^{c} C_{1}^{c}-2 B_{1}^{c} C_{1}^{c}+A_{1}^{c} D_{1}^{c}+A_{1}^{c} \bar{\varphi}_{c, 1}^{(1)}\right),
\end{aligned}
$$




$$
\begin{aligned}
& \tilde{\Delta}_{c}^{34}=\Delta_{c}^{34}+\frac{3}{2}\left(A_{1}^{c}\right)^{2} \zeta_{2} C_{1}^{c}, \\
& \tilde{\Delta}_{c}^{45}=\Delta_{c}^{45}+\frac{1}{8}\left(A_{1}^{c}\right)^{2} \zeta_{2}\left(-10 C_{1}^{c} \beta_{0}-12 f_{1}^{c} C_{1}^{c}-12 B_{1}^{c} C_{1}^{c}+3 A_{1}^{c} D_{1}^{c}+3 A_{1}^{c} \bar{\varphi}_{c, 1}^{(1)}\right), \\
& \tilde{\Delta}_{c}^{46}=\Delta_{c}^{46}+\frac{3}{8}\left(A_{1}^{c}\right)^{3} \zeta_{2} C_{1}^{c} .
\end{aligned}
$$

The expressions for $\tilde{\Delta}_{c}^{10}, \tilde{\Delta}_{c}^{11}, \tilde{\Delta}_{c}^{12}, \tilde{\Delta}_{c}^{23}, \tilde{\Delta}_{c}^{24}, \tilde{\Delta}_{c}^{35}, \tilde{\Delta}_{c}^{36}, \tilde{\Delta}_{c}^{47}$ and $\tilde{\Delta}_{c}^{48}$ are exactly same as the corresponding expressions for the case of DIS. The above results with the explicit dependence on $\mu_{R}^{2}$ and $\mu_{F}^{2}$ are provided in the supplementary material file attached to this paper.

\section{B SV coefficients $\overline{\mathcal{G}}_{S V, i}^{c}$ in $\Delta_{c}$}

We present here the expressions for $\overline{\mathcal{G}}_{S V, i}^{c}$ used in the $\Delta_{c}^{i k}$ for DIS.

$$
\begin{aligned}
\overline{\mathcal{G}}_{S V, 1}^{c} & =\overline{\mathcal{G}}_{1}^{c,(1)}, \\
\overline{\mathcal{G}}_{S V, 2}^{c} & =\left(\overline{\mathcal{G}}_{2}^{c,(1)}+2 \beta_{0} \overline{\mathcal{G}}_{1}^{c,(2)}\right), \\
\overline{\mathcal{G}}_{S V, 3}^{c} & =\left(\frac{2}{3} \overline{\mathcal{G}}^{c,(1)}+\frac{4}{3} \beta_{1} \overline{\mathcal{G}}_{1}^{c,(2)}+\frac{4}{3} \beta_{0} \overline{\mathcal{G}}_{2}^{c,(2)}+\frac{2}{3} \beta_{0}^{2} \overline{\mathcal{G}}_{1}^{c,(3)}\right), \\
\overline{\mathcal{G}}_{S V, 4}^{c} & =\left(\frac{1}{2} \overline{\mathcal{G}}_{4}^{c,(1)}+\beta_{2} \overline{\mathcal{G}}_{1}^{c,(2)}+\beta_{1} \overline{\mathcal{G}}_{2}^{c,(2)}+\beta_{0} \overline{\mathcal{G}}_{3}^{c,(2)}+2 \beta_{0}^{2} \overline{\mathcal{G}}_{2}^{c,(3)}+4 \beta_{0}^{3} \overline{\mathcal{G}}_{1}^{c,(4)}+4 \beta_{0} \beta_{1} \overline{\mathcal{G}}_{1}^{c,(3)}\right),
\end{aligned}
$$

The explicit results for $\overline{\mathcal{G}}_{S V, 1}^{c}, \overline{\mathcal{G}}_{S V, 2}^{c}$ and $\overline{\mathcal{G}}_{S V, 3}^{c}$ in terms of colour factors $C_{A}, C_{F}$ and $n_{f}$ are given in the supplementary material file attached to this paper.

\section{NSV resummation constants $h_{i, j}^{c}(\omega)$}

The resummation constants $h_{i j}^{c}(\omega)$ given in eq. (4.16) are found to be as following. Here $\bar{L}_{\omega}=\log (1-\omega), L_{q r}=\log \left(\frac{Q^{2}}{\mu_{R}^{2}}\right), L_{f r}=\log \left(\frac{\mu_{F}^{2}}{\mu_{R}^{2}}\right)$ and $\omega=\beta_{0} a s\left(\mu_{R}^{2}\right) \log N$.

$$
\begin{aligned}
h_{00}^{c}(\omega)= & \frac{1}{\beta_{0}} \bar{L}_{\omega}\left[\gamma_{1}^{B} D_{1}^{c}-\gamma_{2}^{B} C_{1}^{c}\right], \\
h_{01}^{c}(\omega)= & \frac{1}{\beta_{0}} \bar{L}_{\omega}\left[-\gamma_{1}^{B} C_{1}^{c}\right], \\
h_{10}^{c}(\omega)= & \frac{1}{1-\omega}\left[\bar{\varphi}_{c, 1}^{(0)} \gamma_{1}^{B}-\bar{\varphi}_{c, 1}^{(1)} \gamma_{2}^{B}-\frac{D_{2}^{c}}{\beta_{0}} \gamma_{1}^{B} \omega+\frac{\beta_{1} D_{1}^{c}}{\beta_{0}^{2}} \gamma_{1}^{B}\left\{\omega+\bar{L}_{\omega}\right\}-D_{1}^{c}\left\{\gamma_{2}^{B}-\gamma_{1}^{B} L_{q r}+\gamma_{1}^{B} L_{f r}\right.\right. \\
& \left.-\gamma_{1}^{B} L_{f r} \omega\right\}+\frac{C_{2}^{c}}{\beta_{0}}\left\{\gamma_{2}^{B} \omega\right\}-\frac{\beta_{1} C_{1}^{c}}{\beta_{0}^{2}} \gamma_{2}^{B}\left\{\omega+\bar{L}_{\omega}\right\}+C_{1}^{c}\left\{2 \gamma_{3}^{B}-\gamma_{2}^{B} L_{q r}\right. \\
& \left.\left.+\gamma_{2}^{B} L_{f r}-\gamma_{2}^{B} L_{f r} \omega\right\}\right],
\end{aligned}
$$




$$
\begin{aligned}
h_{11}^{c}(\omega)= & \frac{1}{1-\omega}\left[-\bar{\varphi}_{c, 1}^{(1)} \gamma_{1}^{B}+\frac{C_{2}^{c}}{\beta_{0}}\left\{\gamma_{1}^{B} \omega\right\}-\frac{\beta_{1} C_{1}^{c}}{\beta_{0}^{2}} \gamma_{1}^{B}\left\{\omega+\bar{L}_{\omega}\right\}+C_{1}^{c}\left\{\gamma_{2}^{B}-\gamma_{1}^{B} L_{q r}\right.\right. \\
& \left.\left.+\gamma_{1}^{B} L_{f r}-\gamma_{1}^{B} L_{f r} \omega\right\}\right], \\
h_{21}^{c}(\omega)= & \frac{1}{(1-\omega)^{2}}\left[\frac{\beta_{1}}{\beta_{0}} \bar{\varphi}_{c, 1}^{(1)}\left\{\gamma_{1}^{B} \bar{L}_{\omega}\right\}+2 \bar{\varphi}_{c, 2}^{(2)} \gamma_{2}^{B}-\bar{\varphi}_{c, 2}^{(1)} \gamma_{1}^{B}-\beta_{0} \bar{\varphi}_{c, 1}^{(1)}\left\{\gamma_{2}^{B}-\gamma_{1}^{B} L_{q r}\right\}+\frac{C_{3}^{c}}{2 \beta_{0}} \gamma_{1}^{B}\{2 \omega\right. \\
& \left.-\omega^{2}\right\}-\frac{\beta_{1} C_{2}^{c}}{2 \beta_{0}^{2}} \gamma_{1}^{B}\left\{2 \omega-\omega^{2}+2 \bar{L}_{\omega}\right\}+C_{2}^{c}\left\{\gamma_{2}^{B}-\gamma_{1}^{B} L_{q r}+\gamma_{1}^{B} L_{f r}-2 \gamma_{1}^{B} L_{f r} \omega\right. \\
& \left.+\gamma_{1}^{B} L_{f r} \omega^{2}\right\}-\frac{\beta_{1}^{2} C_{1}^{c}}{2 \beta_{0}^{3}} \gamma_{1}^{B}\left\{\omega^{2}-\bar{L}_{\omega}^{2}\right\}+\frac{\beta_{2} C_{1}^{c}}{2 \beta_{0}^{2}}\left\{\gamma_{1}^{B} \omega^{2}\right\}-\frac{\beta_{1} C_{1}^{c}}{\beta_{0}}\left\{\gamma_{2}^{B} \bar{L}_{\omega}-\gamma_{1}^{B} L_{q r} \bar{L}_{\omega}\right\} \\
& \left.+\frac{C_{1}^{c} \beta_{0}}{2}\left\{2 \gamma_{3}^{B}-2 \gamma_{2}^{B} L_{q r}+\gamma_{1}^{B} L_{q r}^{2}-\gamma_{1}^{B} L_{f r}^{2}+2 \gamma_{1}^{B} L_{f r}^{2} \omega-\gamma_{1}^{B} L_{f r}^{2} \omega^{2}\right\}\right], \\
h_{22}^{c}(\omega)= & \frac{1}{(1-\omega)^{2}}\left[\bar{\varphi}_{c, 2}^{(2)} \gamma_{1}^{B}\right], \\
& \left.\left.-2 \gamma_{2}^{B} L_{q r}+\gamma_{1}^{B} L_{q r}^{2}\right\}\right], \\
h_{32}^{c}(\omega)= & \frac{1}{(1-\omega)^{3}}\left[\frac{\beta_{1}}{\beta_{0}} \bar{\varphi}_{c, 2}^{(2)}\left\{-2 \gamma_{1}^{B} \bar{L}_{\omega}\right\}-3 \bar{\varphi}_{c, 3}^{(3)} \gamma_{2}^{B}+\bar{\varphi}_{c, 3}^{(2)} \gamma_{1}^{B}+2 \beta_{0} \bar{\varphi}_{c, 2}^{(2)}\left\{\gamma_{2}^{B}-\gamma_{1}^{B} L_{q r}\right\}\right], \\
& -\frac{3 \beta_{1}}{\beta_{0}} \bar{\varphi}_{c, 3}^{(2)} \gamma_{1}^{B} \bar{L}_{\omega}+12 \bar{\varphi}_{c, 4}^{(4)} \gamma_{3}^{B}-3 \bar{\varphi}_{c, 4}^{(3)} \gamma_{2}^{B}+\bar{\varphi}_{c, 4}^{(2)} \gamma_{1}^{B}+2 \beta_{1} \bar{\varphi}_{c, 2}^{(2)}\left\{\gamma_{2}^{B}-3 \gamma_{2}^{B} \bar{L}_{\omega}-\gamma_{1}^{B} L_{q r}(3)\left\{2 \gamma_{3}^{B}-\gamma_{2}^{B} L_{q r}\right\}+3 \beta_{0} \bar{\varphi}_{c, 3}^{(2)}\left\{\gamma_{2}^{B}-\gamma_{1}^{B} L_{q r}\right\}+3 \beta_{0}^{2} \bar{\varphi}_{c, 2}^{(2)}\left\{2 \gamma_{3}^{B}\right.\right. \\
h_{33}^{c}(\omega)= & \frac{1}{(1-\omega)^{3}}\left[-\bar{\varphi}_{c, 3}^{(3)} \gamma_{1}^{B}\right], \\
h_{44}^{c}(\omega)= & \left.\frac{1}{(1-\omega)^{4}}\left[\frac{3 \beta_{1}}{\beta_{0}} \bar{\varphi}_{c, 3}^{(3)} \gamma_{1}^{B} \bar{\varphi}_{\omega, 4}^{(4)} \gamma_{1}^{B}\right] .4 \bar{\varphi}_{c, 4}^{(4)} \gamma_{2}^{B}-\bar{\varphi}_{c, 4}^{(3)} \gamma_{1}^{B}-3 \beta_{0} \bar{\varphi}_{c, 3}^{(3)}\left\{\gamma_{2}^{B}-\gamma_{1}^{B} L_{q r}\right\}\right], \\
h_{42}^{c}(\omega)= & \frac{1}{(1-\omega)^{4}}\left[\frac{\beta_{1}^{2}}{\beta_{0}^{(2)}} \bar{\varphi}_{c, 2}\left\{-2 \gamma_{1}^{B} \omega-2 \gamma_{1}^{B} \bar{L}_{\omega}+3 \gamma_{1}^{B} \bar{L}_{\omega}^{2}\right\}+\frac{2 \beta_{2}}{\beta_{0}} \bar{\varphi}_{c, 2}^{(2)} \gamma_{1}^{B} \omega+\frac{9 \beta_{1}}{\beta_{0}} \bar{\varphi}_{c, 3}^{(3)} \gamma_{2}^{B} \bar{L}_{\omega}\right.
\end{aligned}
$$

The above results along with the bigger ones $\left(h_{20}^{c}(\omega), h_{30}^{c}(\omega), h_{31}^{c}(\omega)\right)$ and $\left(h_{40}^{c}(\omega), h_{41}^{c}(\omega)\right)$ are all provided in the supplementary material file attached to this paper.

\section{NSV resummation constants $\bar{g}_{i}^{c}(\omega)$}

The resummation constants $\bar{g}_{i}^{c}(\omega)$ given in eq. (4.16) are presented below. Here $\bar{L}_{\omega}=$ $\log (1-\omega), L_{q r}=\log \left(\frac{Q^{2}}{\mu_{R}^{2}}\right), L_{f r}=\log \left(\frac{\mu_{F}^{2}}{\mu_{R}^{2}}\right)$ and $\omega=\beta_{0} a_{s}\left(\mu_{R}^{2}\right) \log (N)$. Also, $\mathbf{B}_{D I S, i}^{c}$ are the 
threshold exponent given in [45].

$$
\begin{aligned}
& \bar{g}_{2}^{c}(\omega)=\frac{A_{1}^{c}}{2 \beta_{0}} \bar{L}_{\omega}, \\
& \bar{g}_{3}^{c}(\omega)=\frac{1}{(1-\omega)}\left[-\frac{A_{2}^{c} \omega}{2 \beta_{0}}+\frac{\beta_{1} A_{1}^{c}}{2 \beta_{0}^{2}}\left\{\omega+\bar{L}_{\omega}\right\}-\frac{A_{1}^{c}}{2}\left\{1+\gamma_{E}-L_{q r}+L_{f r}-L_{f r} \omega\right\}+\frac{\mathbf{B}_{D I S, 1}^{c}}{2}\right], \\
& \bar{g}_{4}^{c}(\omega)=\frac{1}{(1-\omega)^{2}}\left[-\frac{\beta_{1}}{2 \beta_{0}} \mathbf{B}_{D I S, 1}^{c} \bar{L}_{\omega}+\frac{\beta_{0}}{2} \mathbf{B}_{D I S, 1}^{c}\left\{1+\gamma_{E}-L_{q r}\right\}-\frac{A_{3}^{c}}{4 \beta_{0}}\left\{2 \omega-\omega^{2}\right\}+\frac{\beta_{1} A_{2}^{c}}{4 \beta_{0}^{2}}\{2 \omega\right. \\
& \left.-\omega^{2}+2 \bar{L}_{\omega}\right\}-\frac{A_{2}^{c}}{2}\left\{1+\gamma_{E}-L_{q r}+L_{f r}-2 L_{f r} \omega+L_{f r} \omega^{2}\right\}+\frac{\beta_{1}^{2} A_{1}^{c}}{4 \beta_{0}^{3}}\left\{\omega^{2}-\bar{L}_{\omega}^{2}\right\}-\frac{\beta_{2} A_{1}^{c}}{4 \beta_{0}^{2}}\{ \\
& \left.\omega^{2}\right\}+\frac{\beta_{1} A_{1}^{c}}{2 \beta_{0}}\left\{\bar{L}_{\omega}+\bar{L}_{\omega} \gamma_{E}-L_{q r} \bar{L}_{\omega}\right\}-\frac{\beta_{0} A_{1}^{c}}{4}\left\{2 \gamma_{E}+\gamma_{E}^{2}+\zeta_{2}-2 L_{q r}-2 L_{q r} \gamma_{E}+L_{q r}^{2}-L_{f r}^{2}\right. \\
& \left.\left.+2 L_{f r}^{2} \omega-L_{f r}^{2} \omega^{2}\right\}+\frac{\mathbf{B}_{D I S, 2}^{c}}{2}\right] \text {, } \\
& \bar{g}_{5}^{c}(\omega)=\frac{1}{(1-\omega)^{3}}\left[-\frac{\beta_{1}^{2} \mathbf{B}_{D I S, 1}^{c}}{2 \beta_{0}^{2}}\left\{\omega+\bar{L}_{\omega}-\bar{L}_{\omega}^{2}\right\}+\frac{\beta_{2}}{2 \beta_{0}} \omega \mathbf{B}_{D I S, 1}^{c}-\frac{\beta_{1}}{\beta_{0}} \bar{L}_{\omega} \mathbf{B}_{D I S, 2}^{c}+\frac{\beta_{1} \mathbf{B}_{D I S, 1}^{c}}{2}\{1\right. \\
& \left.+\gamma_{E}-2 \bar{L}_{\omega}-2 \bar{L}_{\omega} \gamma_{E}-L_{q r}+2 L_{q r} \bar{L}_{\omega}\right\}+\beta_{0} \mathbf{B}_{D I S, 2}^{c}\left\{1+\gamma_{E}-L_{q r}\right\}+\frac{\beta_{0}^{2} \mathbf{B}_{D I S, 1}^{c}}{2}\left\{2 \gamma_{E}\right. \\
& \left.+\gamma_{E}^{2}+\zeta_{2}-2 L_{q r}-2 L_{q r} \gamma_{E}+L_{q r}^{2}\right\}-\frac{A_{4}^{c}}{6 \beta_{0}}\left\{3 \omega-3 \omega^{2}+\omega^{3}\right\}+\frac{\beta_{1} A_{3}^{c}}{6 \beta_{0}^{2}}\left\{3 \omega-3 \omega^{2}+\omega^{3}\right. \\
& \left.+3 \bar{L}_{\omega}\right\}-\frac{A_{3}^{c}}{2}\left\{1+\gamma_{E}-L_{q r}+L_{f r}-3 L_{f r} \omega+3 L_{f r} \omega^{2}-L_{f r} \omega^{3}\right\}+\frac{\beta_{1}^{2} A_{2}^{c}}{6 \beta_{0}^{3}}\left\{3 \omega^{2}-\omega^{3}\right. \\
& \left.-3 \bar{L}_{\omega}^{2}\right\}-\frac{\beta_{2} A_{2}^{c}}{6 \beta_{0}^{2}}\left\{3 \omega^{2}-\omega^{3}\right\}+\frac{\beta_{1} A_{2}^{c} \bar{L}_{\omega}}{\beta_{0}}\left\{1+\gamma_{E}-L_{q r}\right\}-\frac{A_{2}^{c} \beta_{0}}{2}\left\{2 \gamma_{E}+\gamma_{E}^{2}+\zeta_{2}-2 L_{q r}\right. \\
& \left.-2 L_{q r} \gamma_{E}+L_{q r}^{2}-L_{f r}^{2}+3 L_{f r}^{2} \omega-3 L_{f r}^{2} \omega^{2}+L_{f r}^{2} \omega^{3}\right\}-\frac{\beta_{1}^{3} A_{1}^{c}}{12 \beta_{0}^{4}}\left\{3 \omega^{2}-2 \omega^{3}+6 \bar{L}_{\omega} \omega+3 \bar{L}_{\omega}^{2}\right. \\
& \left.-2 \bar{L}_{\omega}^{3}\right\}+\frac{\beta_{1} \beta_{2} A_{1}^{c}}{6 \beta_{0}^{3}}\left\{3 \omega^{2}-2 \omega^{3}+3 \bar{L}_{\omega} \omega\right\}-\frac{\beta_{3} A_{1}^{c}}{12 \beta_{0}^{2}}\left\{3 \omega^{2}-2 \omega^{3}\right\}+\frac{\beta_{1}^{2} A_{1}^{c}}{2 \beta_{0}^{2}}\left\{\omega+\omega \gamma_{E}+\bar{L}_{\omega}\right. \\
& \left.+\bar{L}_{\omega} \gamma_{E}-\bar{L}_{\omega}^{2}-\bar{L}_{\omega}^{2} \gamma_{E}-L_{q r} \omega-L_{q r} \bar{L}_{\omega}+L_{q r} \bar{L}_{\omega}^{2}\right\}-\frac{\beta_{2} A_{1}^{c}}{2 \beta_{0}}\left\{\omega+\omega \gamma_{E}-L_{q r} \omega\right\}-\frac{A_{1}^{c} \beta_{1}}{4}\{ \\
& 2 \gamma_{E}+\gamma_{E}^{2}+\zeta_{2}-4 \bar{L}_{\omega} \gamma_{E}-2 \bar{L}_{\omega} \gamma_{E}^{2}-2 \bar{L}_{\omega} \zeta_{2}-2 L_{q r}-2 L_{q r} \gamma_{E}+4 L_{q r} \bar{L}_{\omega}+4 L_{q r} \bar{L}_{\omega} \gamma_{E}+L_{q r}^{2} \\
& \left.-2 L_{q r}^{2} \bar{L}_{\omega}-L_{f r}^{2}+3 L_{f r}^{2} \omega-3 L_{f r}^{2} \omega^{2}+L_{f r}^{2} \omega^{3}\right\}-\frac{A_{1}^{c} \beta_{0}^{2}}{6}\left\{3 \gamma_{E}^{2}+\gamma_{E}^{3}+2 \zeta_{3}+3 \zeta_{2}+3 \zeta_{2} \gamma_{E}\right. \\
& \left.-6 L_{q r} \gamma_{E}-3 L_{q r} \gamma_{E}^{2}-3 L_{q r} \zeta_{2}+3 L_{q r}^{2}+3 L_{q r}^{2} \gamma_{E}-L_{q r}^{3}+L_{f r}^{3}-3 L_{f r}^{3} \omega+3 L_{f r}^{3} \omega^{2}-L_{f r}^{3} \omega^{3}\right\} \\
& \left.+\frac{\mathbf{B}_{D I S, 3}^{c}}{2}\right] \text {. }
\end{aligned}
$$

As before here also we provide the above results along with $\bar{g}_{6}^{c}(\omega)$ in the supplementary material file attached to this paper.

Open Access. This article is distributed under the terms of the Creative Commons Attribution License (CC-BY 4.0), which permits any use, distribution and reproduction in any medium, provided the original author(s) and source are credited. 


\section{References}

[1] T. Kinoshita, Mass singularities of Feynman amplitudes, J. Math. Phys. 3 (1962) 650 [INSPIRE].

[2] T.D. Lee and M. Nauenberg, Degenerate Systems and Mass Singularities, Phys. Rev. 133 (1964) B1549 [INSPIRE].

[3] J.A.M. Vermaseren, A. Vogt and S. Moch, The Third-order QCD corrections to deep-inelastic scattering by photon exchange, Nucl. Phys. B 724 (2005) 3 [hep-ph/0504242] [INSPIRE].

[4] G. Soar, S. Moch, J.A.M. Vermaseren and A. Vogt, On Higgs-exchange DIS, physical evolution kernels and fourth-order splitting functions at large x, Nucl. Phys. B $\mathbf{8 3 2}$ (2010) 152 [arXiv: 0912.0369] [INSPIRE].

[5] J. Ablinger et al., The 3-Loop Non-Singlet Heavy Flavor Contributions and Anomalous Dimensions for the Structure Function $F_{2}\left(x, Q^{2}\right)$ and Transversity, Nucl. Phys. B 886 (2014) 733 [arXiv: 1406.4654] [INSPIRE].

[6] S. Catani, The Singular behavior of QCD amplitudes at two loop order, Phys. Lett. B 427 (1998) 161 [hep-ph/9802439] [INSPIRE].

[7] T. Becher and M. Neubert, Infrared singularities of scattering amplitudes in perturbative QCD, Phys. Rev. Lett. 102 (2009) 162001 [Erratum ibid. 111 (2013) 199905] [arXiv: 0901.0722] [INSPIRE].

[8] T. Becher and M. Neubert, On the Structure of Infrared Singularities of Gauge-Theory Amplitudes, JHEP 06 (2009) 081 [Erratum ibid. 11 (2013) 024] [arXiv:0903.1126] [INSPIRE].

[9] E. Gardi and L. Magnea, Factorization constraints for soft anomalous dimensions in QCD scattering amplitudes, JHEP 03 (2009) 079 [arXiv:0901.1091] [INSPIRE].

[10] A.H. Ajjath, P. Mukherjee and V. Ravindran, Infrared structure of $\mathrm{SU}(N) \times U(1)$ gauge theory to three loops, JHEP 08 (2020) 156 [arXiv: 1912.13386] [INSPIRE].

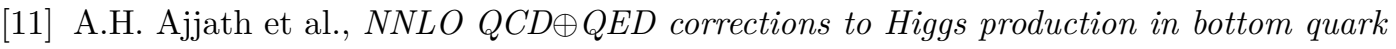
annihilation, Phys. Rev. D 100 (2019) 114016 [arXiv:1906.09028] [INSPIRE].

[12] C. Anastasiou, C. Duhr, F. Dulat, F. Herzog and B. Mistlberger, Higgs Boson Gluon-Fusion Production in QCD at Three Loops, Phys. Rev. Lett. 114 (2015) 212001 [arXiv: 1503. 06056] [INSPIRE].

[13] B. Mistlberger, Higgs boson production at hadron colliders at $N^{3} L O$ in $Q C D$, JHEP 05 (2018) 028 [arXiv: 1802.00833] [inSPIRE].

[14] C. Duhr, F. Dulat and B. Mistlberger, Higgs Boson Production in Bottom-Quark Fusion to Third Order in the Strong Coupling, Phys. Rev. Lett. 125 (2020) 051804 [arXiv: 1904.09990] [INSPIRE].

[15] R. Hamberg, W.L. van Neerven and T. Matsuura, A complete calculation of the order $\$ \alpha_{s}^{2}$ correction to the Drell-Yan K factor, Nucl. Phys. B 359 (1991) 343 [Erratum ibid. 644 (2002) 403] [INSPIRE].

[16] R.V. Harlander and W.B. Kilgore, Next-to-next-to-leading order Higgs production at hadron colliders, Phys. Rev. Lett. 88 (2002) 201801 [hep-ph/0201206] [INSPIRE]. 
[17] C. Duhr, F. Dulat and B. Mistlberger, Drell-Yan Cross Section to Third Order in the Strong Coupling Constant, Phys. Rev. Lett. 125 (2020) 172001 [arXiv:2001.07717] [InSPIRE].

[18] H.M. Georgi, S.L. Glashow, M.E. Machacek and D.V. Nanopoulos, Higgs Bosons from Two Gluon Annihilation in Proton Proton Collisions, Phys. Rev. Lett. 40 (1978) 692 [InSPIRE].

[19] D. Graudenz, M. Spira and P.M. Zerwas, QCD corrections to Higgs boson production at proton proton colliders, Phys. Rev. Lett. 70 (1993) 1372 [INSPIRE].

[20] A. Djouadi, M. Spira and P.M. Zerwas, Production of Higgs bosons in proton colliders: QCD corrections, Phys. Lett. B 264 (1991) 440 [INSPIRE].

[21] M. Spira, A. Djouadi, D. Graudenz and P.M. Zerwas, Higgs boson production at the LHC, Nucl. Phys. B 453 (1995) 17 [hep-ph/9504378] [InSPIRE].

[22] S. Catani, D. de Florian and M. Grazzini, Higgs production in hadron collisions: Soft and virtual QCD corrections at NNLO, JHEP 05 (2001) 025 [hep-ph/0102227] [INSPIRE].

[23] R.V. Harlander and W.B. Kilgore, Soft and virtual corrections to proton proton $\rightarrow H+x$ at NNLO, Phys. Rev. D 64 (2001) 013015 [hep-ph/0102241] [INSPIRE].

[24] C. Anastasiou and K. Melnikov, Higgs boson production at hadron colliders in NNLO QCD, Nucl. Phys. B 646 (2002) 220 [hep-ph/0207004] [INSPIRE].

[25] S. Catani, D. de Florian, M. Grazzini and P. Nason, Soft gluon resummation for Higgs boson production at hadron colliders, JHEP 07 (2003) 028 [hep-ph/0306211] [INSPIRE].

[26] V. Ravindran, J. Smith and W.L. van Neerven, NNLO corrections to the total cross-section for Higgs boson production in hadron hadron collisions, Nucl. Phys. B 665 (2003) 325 [hep-ph/0302135] [INSPIRE].

[27] S. Moch and A. Vogt, Higher-order soft corrections to lepton pair and Higgs boson production, Phys. Lett. B 631 (2005) 48 [hep-ph/0508265] [INSPIRE].

[28] V. Ravindran, Higher-order threshold effects to inclusive processes in QCD, Nucl. Phys. B 752 (2006) 173 [hep-ph/0603041] [INSPIRE].

[29] D. de Florian and J. Mazzitelli, A next-to-next-to-leading order calculation of soft-virtual cross sections, JHEP 12 (2012) 088 [arXiv:1209.0673] [INSPIRE].

[30] M. Bonvini, R.D. Ball, S. Forte, S. Marzani and G. Ridolfi, Updated Higgs cross section at approximate $N^{3} L O$, J. Phys. G 41 (2014) 095002 [arXiv:1404.3204] [INSPIRE].

[31] D. de Florian, J. Mazzitelli, S. Moch and A. Vogt, Approximate $N^{3}$ LO Higgs-boson production cross section using physical-kernel constraints, JHEP 10 (2014) 176 [arXiv: 1408.6277] [INSPIRE].

[32] C. Anastasiou et al., Higgs boson gluon-fusion production at threshold in $N^{3} L O Q C D$, Phys. Lett. B 737 (2014) 325 [arXiv:1403.4616] [INSPIRE].

[33] Y. Li, A. von Manteuffel, R.M. Schabinger and H.X. Zhu, Soft-virtual corrections to Higgs production at $N^{3} L O$, Phys. Rev. D 91 (2015) 036008 [arXiv:1412.2771] [INSPIRE].

[34] C. Anastasiou, C. Duhr, F. Dulat, E. Furlan, F. Herzog and B. Mistlberger, Soft expansion of double-real-virtual corrections to Higgs production at $N^{3} L O$, JHEP 08 (2015) 051 [arXiv: 1505. 04110] [INSPIRE].

[35] G. Das, S. Moch and A. Vogt, Approximate four-loop QCD corrections to the Higgs-boson production cross section, Phys. Lett. B 807 (2020) 135546 [arXiv:2004.00563] [INSPIRE]. 
[36] G. Altarelli, R. Ellis and G. Martinelli, Leptoproduction and Drell-Yan Processes Beyond the Leading Approximation in Chromodynamics, Nucl. Phys. B 143 (1978) 521.

[37] G. Altarelli, R.K. Ellis and G. Martinelli, Large Perturbative Corrections to the Drell-Yan Process in QCD, Nucl. Phys. B 157 (1979) 461 [InSPIRE].

[38] T. Matsuura and W.L. van Neerven, Second Order Logarithmic Corrections to the Drell-Yan Cross-section, Z. Phys. C 38 (1988) 623 [InSPIRE].

[39] T. Matsuura, S.C. van der Marck and W.L. van Neerven, The Order $\alpha^{-} s^{2}$ Contribution to the K Factor of the Drell-Yan Process, Phys. Lett. B 211 (1988) 171 [InSPIRE].

[40] T. Matsuura, S.C. van der Marck and W.L. van Neerven, The Calculation of the Second Order Soft and Virtual Contributions to the Drell-Yan Cross-Section, Nucl. Phys. B 319 (1989) 570 [inSPIRE].

[41] T. Matsuura, R. Hamberg and W.L. van Neerven, The Contribution of the Gluon-gluon Subprocess to the Drell-Yan K Factor, Nucl. Phys. B 345 (1990) 331 [InSPIRE].

[42] W.L. van Neerven and E.B. Zijlstra, The $O\left(\alpha_{s}^{2}\right)$ corrected Drell-Yan $K$ factor in the DIS and MS scheme, Nucl. Phys. B 382 (1992) 11 [Erratum ibid. 680 (2004) 513] [INSPIRE].

[43] T. Ahmed, M. Mahakhud, N. Rana and V. Ravindran, Drell-Yan Production at Threshold to Third Order in QCD, Phys. Rev. Lett. 113 (2014) 112002 [arXiv:1404.0366] [InSPIRE].

[44] S. Catani, L. Cieri, D. de Florian, G. Ferrera and M. Grazzini, Threshold resummation at $N^{3} L L$ accuracy and soft-virtual cross sections at $N^{3} L O$, Nucl. Phys. B 888 (2014) 75 [arXiv: 1405.4827] [INSPIRE].

[45] V. Ravindran, On Sudakov and soft resummations in QCD, Nucl. Phys. B 746 (2006) 58 [hep-ph/0512249] [INSPIRE].

[46] T. Ahmed, N. Rana and V. Ravindran, Higgs boson production through $b \bar{b}$ annihilation at threshold in $N^{3} L O$ QCD, JHEP 10 (2014) 139 [arXiv:1408.0787] [INSPIRE].

[47] M.C. Kumar, M.K. Mandal and V. Ravindran, Associated production of Higgs boson with vector boson at threshold $N^{3} L O$ in QCD, JHEP 03 (2015) 037 [arXiv:1412.3357] [INSPIRE].

[48] Y. Li, A. von Manteuffel, R.M. Schabinger and H.X. Zhu, $N^{3} L O$ Higgs boson and Drell-Yan production at threshold: The one-loop two-emission contribution, Phys. Rev. D 90 (2014) 053006 [arXiv: 1404.5839] [INSPIRE].

[49] G.F. Sterman, Summation of Large Corrections to Short Distance Hadronic Cross-Sections, Nucl. Phys. B 281 (1987) 310 [INSPIRE].

[50] S. Catani and L. Trentadue, Resummation of the QCD Perturbative Series for Hard Processes, Nucl. Phys. B 327 (1989) 323 [InSPIRE].

[51] S. Catani, M.L. Mangano, P. Nason and L. Trentadue, The Resummation of soft gluons in hadronic collisions, Nucl. Phys. B 478 (1996) 273 [hep-ph/9604351] [INSPIRE].

[52] S. Moch, J.A.M. Vermaseren and A. Vogt, Higher-order corrections in threshold resummation, Nucl. Phys. B 726 (2005) 317 [hep-ph/0506288] [INSPIRE].

[53] M. Bonvini, S. Forte and G. Ridolfi, The Threshold region for Higgs production in gluon fusion, Phys. Rev. Lett. 109 (2012) 102002 [arXiv: 1204.5473] [INSPIRE].

[54] M. Bonvini and S. Marzani, Resummed Higgs cross section at $N^{3} L L$, JHEP 09 (2014) 007 [arXiv: 1405.3654] [INSPIRE]. 
[55] M. Bonvini and L. Rottoli, Three loop soft function for $N^{3} L L^{\prime}$ gluon fusion Higgs production in soft-collinear effective theory, Phys. Rev. D 91 (2015) 051301 [arXiv:1412.3791] [INSPIRE].

[56] M. Bonvini, S. Marzani, C. Muselli and L. Rottoli, On the Higgs cross section at $N^{3} L O+N^{3} L L$ and its uncertainty, JHEP 08 (2016) 105 [arXiv: 1603.08000] [INSPIRE].

[57] M. Bonvini, A.S. Papanastasiou and F.J. Tackmann, Matched predictions for the $b \bar{b} H$ cross section at the $13 \mathrm{TeV}$ LHC, JHEP 10 (2016) 053 [arXiv:1605.01733] [INSPIRE].

[58] A.H. Ajjath, A. Chakraborty, G. Das, P. Mukherjee and V. Ravindran, Resummed prediction for Higgs boson production through b $\overline{\mathrm{b}}$ annihilation at $N^{3} L L$, JHEP 11 (2019) 006 [arXiv: 1905.03771] [INSPIRE].

[59] M. Bonvini, Threshold resummation for Drell-Yan production: Theory and phenomenology, PoS DIS2010 (2010) 100 [arXiv: 1006.5918] [InSPIRE].

[60] M. Bonvini, Resummation of soft and hard gluon radiation in perturbative QCD, Ph.D. thesis, Genoa U., 2012. arXiv:1212.0480 [inSPIRE].

[61] A.H. Ajjath, G. Das, M.C. Kumar, P. Mukherjee, V. Ravindran and K. Samanta, Resummed Drell-Yan cross-section at $N^{3} L L$, JHEP 10 (2020) 153 [arXiv:2001.11377] [INSPIRE].

[62] M. Cacciari and S. Catani, Soft gluon resummation for the fragmentation of light and heavy quarks at large x, Nucl. Phys. B 617 (2001) 253 [hep-ph/0107138] [INSPIRE].

[63] S. Moch and A. Vogt, On non-singlet physical evolution kernels and large-x coefficient functions in perturbative QCD, JHEP 11 (2009) 099 [arXiv: 0909.2124] [INSPIRE].

[64] G. Grunberg and V. Ravindran, On threshold resummation beyond leading 1-x order, JHEP 10 (2009) 055 [arXiv: 0902.2702] [INSPIRE].

[65] E. Laenen, L. Magnea and G. Stavenga, On next-to-eikonal corrections to threshold resummation for the Drell-Yan and DIS cross sections, Phys. Lett. B 669 (2008) 173 [arXiv: 0807.4412] [INSPIRE].

[66] E. Laenen, L. Magnea, G. Stavenga and C.D. White, On Next-to-Eikonal Exponentiation, Nucl. Phys. B Proc. Suppl. 205-206 (2010) 260 [arXiv:1007.0624] [inSPIRE].

[67] E. Laenen, L. Magnea, G. Stavenga and C.D. White, Next-to-Eikonal Corrections to Soft Gluon Radiation: A Diagrammatic Approach, JHEP 01 (2011) 141 [arXiv:1010.1860] [INSPIRE].

[68] D. Bonocore, E. Laenen, L. Magnea, L. Vernazza and C.D. White, The method of regions and next-to-soft corrections in Drell-Yan production, Phys. Lett. B 742 (2015) 375 [arXiv:1410.6406] [INSPIRE].

[69] D. Bonocore, E. Laenen, L. Magnea, S. Melville, L. Vernazza and C.D. White, A factorization approach to next-to-leading-power threshold logarithms, JHEP 06 (2015) 008 [arXiv: 1503.05156] [INSPIRE].

[70] M. Beneke, A. Broggio, S. Jaskiewicz and L. Vernazza, Threshold factorization of the Drell-Yan process at next-to-leading power, JHEP 07 (2020) 078 [arXiv:1912.01585] [INSPIRE].

[71] M. Beneke, M. Garny, S. Jaskiewicz, R. Szafron, L. Vernazza and J. Wang, Leading-logarithmic threshold resummation of Higgs production in gluon fusion at next-to-leading power, JHEP 01 (2020) 094 [arXiv:1910.12685] [INSPIRE]. 
[72] D. Bonocore, E. Laenen, L. Magnea, L. Vernazza and C.D. White, Non-abelian factorisation for next-to-leading-power threshold logarithms, JHEP 12 (2016) 121 [arXiv: 1610.06842] [INSPIRE].

[73] V. Del Duca, E. Laenen, L. Magnea, L. Vernazza and C.D. White, Universality of next-to-leading power threshold effects for colourless final states in hadronic collisions, JHEP 11 (2017) 057 [arXiv:1706.04018] [inSPIRE].

[74] A.H. Ajjath, P. Mukherjee and V. Ravindran, On next to soft corrections to Drell-Yan and Higgs Boson productions, arXiv:2006.06726 [INSPIRE].

[75] G. Altarelli and G. Parisi, Asymptotic Freedom in Parton Language, Nucl. Phys. B 126 (1977) 298 [INSPIRE].

[76] S. Moch, J.A.M. Vermaseren and A. Vogt, The Three loop splitting functions in QCD: The Nonsinglet case, Nucl. Phys. B 688 (2004) 101 [hep-ph/0403192] [INSPIRE].

[77] A. Vogt, S. Moch and J.A.M. Vermaseren, The Three-loop splitting functions in QCD: The Singlet case, Nucl. Phys. B 691 (2004) 129 [hep-ph/0404111] [InSPIRE].

[78] A. Gonzalez-Arroyo, C. Lopez and F.J. Yndurain, Second Order Contributions to the Structure Functions in Deep Inelastic Scattering. 1. Theoretical Calculations, Nucl. Phys. B 153 (1979) 161 [INSPIRE].

[79] G. Curci, W. Furmanski and R. Petronzio, Evolution of Parton Densities Beyond Leading Order: The Nonsinglet Case, Nucl. Phys. B 175 (1980) 27 [INSPIRE].

[80] W. Furmanski and R. Petronzio, Singlet Parton Densities Beyond Leading Order, Phys. Lett. B 97 (1980) 437 [inSPIRE].

[81] R. Hamberg and W.L. van Neerven, The Correct renormalization of the gluon operator in a covariant gauge, Nucl. Phys. B 379 (1992) 143 [InSPIRE].

[82] R.K. Ellis and W. Vogelsang, The Evolution of parton distributions beyond leading order: The Singlet case, hep-ph/9602356 [INSPIRE].

[83] J. Ablinger, A. Behring, J. Blümlein, A. De Freitas, A. von Manteuffel and C. Schneider, The three-loop splitting functions $P_{q g}^{(2)}$ and $P_{g g}^{\left(2, N_{F}\right)}$, Nucl. Phys. B $922(2017) 1$ [arXiv: 1705. 01508] [INSPIRE].

[84] S. Moch, B. Ruijl, T. Ueda, J.A.M. Vermaseren and A. Vogt, Four-Loop Non-Singlet Splitting Functions in the Planar Limit and Beyond, JHEP 10 (2017) 041 [arXiv:1707.08315] [INSPIRE].

[85] K.G. Chetyrkin, J.H. Kuhn and C. Sturm, QCD decoupling at four loops, Nucl. Phys. B 744 (2006) 121 [hep-ph/0512060] [INSPIRE].

[86] W.L. van Neerven, Dimensional Regularization of Mass and Infrared Singularities in Two Loop On-shell Vertex Functions, Nucl. Phys. B 268 (1986) 453 [InSPIRE].

[87] R.V. Harlander, Virtual corrections to $g g \rightarrow H$ to two loops in the heavy top limit, Phys. Lett. B 492 (2000) 74 [hep-ph/0007289] [INSPIRE].

[88] V. Ravindran, J. Smith and W.L. van Neerven, Two-loop corrections to Higgs boson production, Nucl. Phys. B 704 (2005) 332 [hep-ph/0408315] [INSPIRE].

[89] S. Moch, J.A.M. Vermaseren and A. Vogt, Three-loop results for quark and gluon form-factors, Phys. Lett. B 625 (2005) 245 [hep-ph/0508055] [INSPIRE]. 
[90] T. Gehrmann, T. Huber and D. Maître, Two-loop quark and gluon form-factors in dimensional regularisation, Phys. Lett. B 622 (2005) 295 [hep-ph/0507061] [INSPIRE].

[91] P.A. Baikov, K.G. Chetyrkin, A.V. Smirnov, V.A. Smirnov and M. Steinhauser, Quark and gluon form factors to three loops, Phys. Rev. Lett. 102 (2009) 212002 [arXiv:0902.3519] [INSPIRE].

[92] T. Gehrmann, E.W.N. Glover, T. Huber, N. Ikizlerli and C. Studerus, Calculation of the quark and gluon form factors to three loops in QCD, JHEP 06 (2010) 094 [arXiv: 1004.3653] [INSPIRE].

[93] T. Gehrmann and D. Kara, The Hbb form factor to three loops in QCD, JHEP 09 (2014) 174 [arXiv:1407.8114] [INSPIRE].

[94] A. von Manteuffel and R.M. Schabinger, Quark and gluon form factors to four-loop order in QCD: the $N_{f}^{3}$ contributions, Phys. Rev. D 95 (2017) 034030 [arXiv:1611.00795] [INSPIRE].

[95] J.M. Henn, A.V. Smirnov, V.A. Smirnov and M. Steinhauser, A planar four-loop form factor and cusp anomalous dimension in QCD, JHEP 05 (2016) 066 [arXiv:1604.03126] [INSPIRE].

[96] J.M. Henn, T. Peraro, M. Stahlhofen and P. Wasser, Matter dependence of the four-loop cusp anomalous dimension, Phys. Rev. Lett. 122 (2019) 201602 [arXiv:1901.03693] [INSPIRE].

[97] A. von Manteuffel, E. Panzer and R.M. Schabinger, Cusp and collinear anomalous dimensions in four-loop QCD from form factors, Phys. Rev. Lett. 124 (2020) 162001 [arXiv: 2002.04617] [INSPIRE].

[98] T. Gehrmann, E.W.N. Glover, T. Huber, N. Ikizlerli and C. Studerus, The quark and gluon form factors to three loops in QCD through to O(eps $\left.{ }^{2}\right)$, JHEP 11 (2010) 102 [arXiv: 1010 .4478] [INSPIRE].

[99] V.V. Sudakov, Vertex parts at very high-energies in quantum electrodynamics, Sov. Phys. JETP 3 (1956) 65 [INSPIRE].

[100] A. Sen, Asymptotic Behavior of the Sudakov Form-Factor in QCD, Phys. Rev. D 24 (1981) 3281 [INSPIRE].

[101] J.C. Collins, Sudakov form-factors, Adv. Ser. Direct. High Energy Phys. 5 (1989) 573 [hep-ph/0312336] [INSPIRE].

[102] L. Magnea and G.F. Sterman, Analytic continuation of the Sudakov form-factor in QCD, Phys. Rev. D 42 (1990) 4222 [INSPIRE].

[103] L. Magnea, Analytic resummation for the quark form-factor in QCD, Nucl. Phys. B 593 (2001) 269 [hep-ph/0006255] [INSPIRE].

[104] G.F. Sterman and M.E. Tejeda-Yeomans, Multiloop amplitudes and resummation, Phys. Lett. B 552 (2003) 48 [hep-ph/0210130] [INSPIRE].

[105] S. Moch, J.A.M. Vermaseren and A. Vogt, The Quark form-factor at higher orders, JHEP 08 (2005) 049 [hep-ph/0507039] [INSPIRE].

[106] C.W. Bauer and A.V. Manohar, Shape function effects in $B \rightarrow X(s)$ gamma and $B \rightarrow X(u)$ l anti-nu decays, Phys. Rev. D 70 (2004) 034024 [hep-ph/0312109] [INSPIRE].

[107] S.W. Bosch, B.O. Lange, M. Neubert and G. Paz, Factorization and shape function effects in inclusive B meson decays, Nucl. Phys. B 699 (2004) 335 [hep-ph/0402094] [INSPIRE]. 
[108] T. Becher and M.D. Schwartz, Direct photon production with effective field theory, JHEP 02 (2010) 040 [arXiv:0911.0681] [INSPIRE].

$[109]$ T. Becher and M. Neubert, Toward a NNLO calculation of the $\bar{B} \rightarrow X(s)$ gamma decay rate with a cut on photon energy. II. Two-loop result for the jet function, Phys. Lett. B 637 (2006) 251 [hep-ph/0603140] [INSPIRE].

[110] T. Becher and G. Bell, The gluon jet function at two-loop order, Phys. Lett. B 695 (2011) 252 [arXiv: 1008.1936] [INSPIRE].

[111] R. Brüser, Z.L. Liu and M. Stahlhofen, Three-Loop Quark Jet Function, Phys. Rev. Lett. 121 (2018) 072003 [arXiv: 1804.09722] [INSPIRE].

[112] P. Banerjee, P.K. Dhani and V. Ravindran, Gluon jet function at three loops in QCD, Phys. Rev. D 98 (2018) 094016 [arXiv: 1805.02637] [InSPIRE].

[113] C.W. Bauer, S. Fleming and M.E. Luke, Summing Sudakov logarithms in B $\rightarrow$ X(s gamma) in effective field theory, Phys. Rev. D 63 (2000) 014006 [hep-ph/0005275] [InSPIRE].

[114] C.W. Bauer, S. Fleming, D. Pirjol and I.W. Stewart, An Effective field theory for collinear and soft gluons: Heavy to light decays, Phys. Rev. D 63 (2001) 114020 [hep-ph/0011336] [INSPIRE].

[115] C.W. Bauer and I.W. Stewart, Invariant operators in collinear effective theory, Phys. Lett. $B 516$ (2001) 134 [hep-ph/0107001] [INSPIRE].

[116] C.W. Bauer, D. Pirjol and I.W. Stewart, Soft collinear factorization in effective field theory, Phys. Rev. D 65 (2002) 054022 [hep-ph/0109045] [INSPIRE].

[117] C.W. Bauer, S. Fleming, D. Pirjol, I.Z. Rothstein and I.W. Stewart, Hard scattering factorization from effective field theory, Phys. Rev. D 66 (2002) 014017 [hep-ph/0202088] [INSPIRE].

[118] M. Beneke, A.P. Chapovsky, M. Diehl and T. Feldmann, Soft collinear effective theory and heavy to light currents beyond leading power, Nucl. Phys. B 643 (2002) 431 [hep-ph/0206152] [INSPIRE].

[119] J. Blumlein and V. Ravindran, QCD threshold corrections to Higgs decay and to hadroproduction in $\ell^{+} \ell^{-}$annihilation, Phys. Lett. B 640 (2006) 40 [hep-ph/0605011] [INSPIRE].

[120] Particle Data Group collaboration, Review of Particle Physics, Phys. Lett. B 667 (2008) 1 [INSPIRE].

[121] M. Beneke et al., Leading-logarithmic threshold resummation of the Drell-Yan process at next-to-leading power, JHEP 03 (2019) 043 [arXiv: 1809.10631] [INSPIRE].

[122] N. Bahjat-Abbas et al., Diagrammatic resummation of leading-logarithmic threshold effects at next-to-leading power, JHEP 11 (2019) 002 [arXiv:1905.13710] [INSPIRE].

[123] K.G. Chetyrkin, G. Falcioni, F. Herzog and J.A.M. Vermaseren, Five-loop renormalisation of QCD in covariant gauges, JHEP 10 (2017) 179 [Addendum ibid. 12 (2017) 006] [arXiv: 1709.08541] [INSPIRE].

[124] T. Luthe, A. Maier, P. Marquard and Y. Schröder, The five-loop $\beta$-function for a general gauge group and anomalous dimensions beyond Feynman gauge, JHEP 10 (2017) 166 [arXiv: 1709.07718] [INSPIRE]. 
[125] F. Herzog, B. Ruijl, T. Ueda, J.A.M. Vermaseren and A. Vogt, The five-loop $\beta$-function of Yang-Mills theory with fermions, JHEP 02 (2017) 090 [arXiv: 1701.01404] [INSPIRE].

[126] P.A. Baikov, K.G. Chetyrkin and J.H. Kühn, Five-Loop Running of the QCD coupling constant, Phys. Rev. Lett. 118 (2017) 082002 [arXiv:1606.08659] [INSPIRE].

[127] G. Das, S.-O. Moch and A. Vogt, Soft corrections to inclusive deep-inelastic scattering at four loops and beyond, JHEP 03 (2020) 116 [arXiv:1912.12920] [INSPIRE].

[128] J. Blumlein, V. Ravindran and W.L. van Neerven, On the Drell-Levy-Yan relation to $O\left(\alpha_{s}^{2}\right)$, Nucl. Phys. B 586 (2000) 349 [hep-ph/0004172] [INSPIRE].

[129] E.G. Floratos, R. Lacaze and C. Kounnas, Space and Timelike Cut Vertices in QCD Beyond the Leading Order. 2. The Singlet Sector, Phys. Lett. B 98 (1981) 285 [INSPIRE].

[130] S.D. Drell, D.J. Levy and T.-M. Yan, A Theory of Deep Inelastic Lepton-Nucleon Scattering and Lepton Pair Annihilation Processes. 1., Phys. Rev. 187 (1969) 2159 [INSPIRE].

[131] G. Grunberg, Large-x structure of physical evolution kernels in Deep Inelastic Scattering, Phys. Lett. B 687 (2010) 405 [arXiv:0911.4471] [InSPIRE]. 